\title{
Akt Inhibition Improves the Efficacy of Cabazitaxel Nanomedicine in Preclinical Taxane-Resistant Cancer Models
}

\section{Tongyu Li}

Zhejiang university

Linlin Shi

Zhejiang University

Jianqin Wan

Zhejiang University

Xiaoxiao $\mathrm{Hu}$

Zhejiang University

Wanzhi Chen

Zhejiang University

Hangxiang Wang ( $\nabla$ wanghx@zju.edu.cn )

Zhejiang University https://orcid.org/0000-0001-6370-9728

\section{Research}

Keywords: multidrug resistance, P-glycoprotein, cabazitaxel, prodrug, cancer nanomedicine, synergistic therapeutics

Posted Date: December 2nd, 2020

DOI: https://doi.org/10.21203/rs.3.rs-117705/v1

License: (c) (1) This work is licensed under a Creative Commons Attribution 4.0 International License. Read Full License 


\section{Abstract}

Background: Drug resistance continues to be a major clinical challenge in achieving cures in cancer patients. Cabazitaxel has shown the ability to surmount drug resistance through bypassing the transporter-mediated drug expulsion; however, the substantially high toxicity in patients hampered its clinical application. In addition, upregulation of certain self-defense proteins (e.g, Akt) was reportedly involved in drug resistance, which may further compromise the activity of cabazitaxel. We have previously developed several prodrug-based strategies to deliver nanoparticles encapsulating cabazitaxel derivatives in tumors with enhanced efficacy and improved in vivo tolerability. Therefore, we hypothesized that combing cabazitaxel nanotherapeutics with a pan-Akt inhibitor MK-2206 would synergistically eliminate the resistant cancers with reduced systemic toxicity.

Methods: Activation of Akt in resistant cancers upon cabazitaxel treatment was determined by western blotting assay. The effect of combing MK-2206 with cabazitaxel on cell viability was evaluated by CCK-8 assay. To improve the in vivo biocompatibility, the delivery of potent cabazitaxel was feasibly achieved via the integration of oligolactide-conjugated cabazitaxel into the PEG-b-PLA matrix. Both the synergism and safety of cabazitaxel nanomedicine-based combination were evaluated through a series of in vitro and in vivo experiments, including Western blotting assay, CCK-8 assay, EdU assay, flow cytometry, migration assay, transwell assay, MTD study, myelosuppression study, nude mouse xenograft, and immunostaining analyses.

Results: We found that resistant cells adapted to activate Akt signaling upon cabazitaxel treatment, which potentially discounts the efficacy of cabazitaxel. The addition of MK-2206 reversed this situation and potentiated the activity of cabazitaxel nanomedicine against resistant cells. Mechanistically, suppression of the Akt pathway increased the microtubule-stabilizing effect of cabazitaxel. Their collaboration was demonstrated to maximize the efficacy in a xenograft model bearing paclitaxelresistant tumors. In particular, the nanoformulation substantially improved drug tolerability in animals, and combined treatment with MK-2206 was proven to be safe for synergistic cancer therapy.

Conclusion: The preclinical studies demonstrate the therapeutic efficacy of our binary system consisting of a better-tolerated nanotherapy and a specific pathway modulator against resistant cells, thereby highlighting the potential applications for the clinical treatment of patients with multidrug-resistant malignancies.

\section{Background}

Taxane-based drugs (e.g., paclitaxel and docetaxel) are the frontline treatments for many types of cancer; they promote the polymerization of tubulins, resulting in cancer cell apoptosis [1]. Unfortunately, the clinical use of these drugs has been greatly compromised by the production of drug resistance and insufficient delivery in tumor lesions [2]. To overcome drug resistance, cabazitaxel (commercial formulation Jevtana $\left.{ }^{\circledR}\right)$, a semisynthetic tubulin-binding taxane derivative, was developed as a second- 
generation taxane for clinical use [3]. This agent shows low affinity to P-glycoprotein (P-gp) and therefore has great therapeutic potential to surmount the hurdle of multidrug resistance (MDR) [4]. Its commercial formulation Jevtana ${ }^{\circledR}$ was approved by the US Food and Drug Administration (FDA) in 2010 for the treatment of patients with hormone-refractory metastatic prostate cancers [5]. However, the clinical efficacy of cabazitaxel is generally limited by poor water solubility and dose-limiting toxicities with low safety profiles $[6,7]$.

Currently, different types of biodegradable nanoparticles have been investigated to improve the targeted delivery of taxane-based agents [8]. The most widely used in pharmaceutical delivery are synthetic (co)polyesters, such as poly(ethylene glycol)-block-poly (D,L-lactic acid) (mPEG-b-PLA) or poly(caprolactone) (PCL), with the production of the most successful paradigm Genexol®-PM, a polymeric micelle formulation of paclitaxel that has been approved for clinical practice in Korea [9-12]. However, such noncovalent approaches to the formulation of chemotherapeutics remain largely unsatisfactory, and many nanotherapeutics have failed to show improved patient outcomes [13, 14]. We previously engineered cytotoxic cabazitaxel to oligolactide to make this agent more compatible with the PEG- $b$-PLA-based polymeric platform, thereby conferring the cabazitaxel agent with the ability to stably circulate in the blood and accumulate in tumors after systemic injection [13]. More importantly, such a nanoplatform was expected to increase the maximum tolerated dose of cabazitaxel to better exert therapeutic outcomes in paclitaxel-resistant cancers.

The serine/threonine kinase Akt, comprised of 3 isoforms (Akt 1, 2, and 3), is a key player in the regulatory network of the cell and affects a variety of cellular activities, including cell growth, survival, migration, differentiation, and metabolism $[15,16]$. Previous studies have indicated a correlation between Akt activation and drug resistance. When exposed to paclitaxel or other chemotherapeutics, cancer cells showed upregulation of activated Akt as an adaptive mechanism, thus making cells less responsive to chemotherapeutic drugs [17-19]. Furthermore, Akt is considered an exciting target for therapeutics since Akt inactivation has been demonstrated to be effective in promoting the cytotoxic activity of therapeutic agents against a variety of cancers $[20,21]$. In light of the above knowledge, we presumed that exposure of resistant cells to cabazitaxel may also stimulate the activation of Akt and thereby benefit from additional Akt inhibition.

In this study, we first validated that cabazitaxel treatment promoted the activation of Akt signaling in paclitaxel-resistant cervical cancer cells (HeLa/Taxol) and non-small cell lung cancer (NSCLC) cells (A549/Taxol). Interestingly, Akt activation was reversed by MK-2206, a highly potent and selective Akt inhibitor, resulting in increased cytotoxicity against both cell lines. To ameliorate the side effects of cabazitaxel induced by delivery with the clinical formulation, oligolactide was exploited to covalently conjugate with cabazitaxel and further encapsulate the prodrug in the PEG- $b$-PLA matrix to form injectable nanoparticles (termed cabazitaxel-NPs). Further in vitro and in vivo studies were conducted to investigate the efficacy of the combinatorial regimen for the treatment of resistant cancer. Overall, this study increases our understanding of how to use low toxicity Akt inhibitors to compensate for toxic 
cabazitaxel, aiding further investigation of the therapeutic potential in the treatment of taxane-resistant cancers.

\section{Materials And Methods \\ Materials}

Cabazitaxel (CTX) and D, $_{\text {- }}$-lactide were purchased from Tokyo Chemical Industry Co., Ltd. (Shanghai, China). Methoxypoly (ethylene glycol)-block-poly (D,L-lactide) $\left(\mathrm{mPEG}_{5 \mathrm{k}}-\mathrm{PLA}_{8 \mathrm{k}}\right.$ ) copolymers were obtained from Jinan Daigang Biomaterial Co., Ltd. All other compounds and solvents were purchased from J\&K Chemical (Shanghai, China) and freshly used after unsealing.

\section{Preparation of oligolactide-cabazitaxel-formulated nanoparticles}

OLA-CTX was incorporated into PEG- $b$-PLA micelles using a previously reported nanoprecipitation approach. In brief, $1.0 \mathrm{mg}$ of OLA-CTX (at a CTX equivalence) together with $20.0 \mathrm{mg} \mathrm{PEG} \mathrm{PK}-b-\mathrm{PLA} \mathrm{A}_{8 \mathrm{~K}}$ were dissolved in $1.0 \mathrm{~mL}$ of acetone with ultrasonication; the mixture was gradually dropped into a flask containing $10 \mathrm{~mL}$ of deionized (DI) water while stirring. After stirring at room temperature for $15 \mathrm{~min}$, the excess organic solvent was carefully removed using a rotary evaporator under reduced pressure. The remaining solution containing the micelles was concentrated with centrifugal filter devices (Amicon Ultra4, 10 k MWCO, Millipore Corp.) and rinsed with DI water.

\section{Cell lines and cell culture}

The human NSCLC A549 cell line and cervical cancer HeLa cell line were obtained from the cell bank of the Chinese Academy of Sciences (Shanghai, China). The paclitaxel-resistant A549/Taxol cells were purchased from Shanghai Bogoo Biotechnology with certification, whereas the resistant HeLa/Taxol cells were generated from parental HeLa cells by increasing the concentrations of paclitaxel in the culture medium stepwise, according to previously reported methods. All cells were cultured in RPMI 1640 medium supplemented with $10 \%$ fetal bovine serum and $1 \%$ antibiotic-antimycotic and maintained at $37^{\circ} \mathrm{C}$ in a $5 \% \mathrm{CO}_{2}$ incubator with humidity.

\section{Cytotoxicity study in resistant cells}

Cytotoxicity was evaluated by Counting Kit-8 assays (CCK-8; MedChem Express, Monmouth Junction, NJ, USA). Briefly, cells were seeded into 96 -well plates at a density of $3 \times 10^{3}$ cells per well. After attaching at $37^{\circ} \mathrm{C}$ for $24 \mathrm{~h}$, cells were treated with varying concentrations of CTX, cabazitaxel-NP micelles, or in combination with MK-2206 for another $72 \mathrm{~h}$. Then, cells were treated with fresh media containing $10 \%$ CCK-8 solution for 1-2 $\mathrm{h}$. The cell viability was determined by reading the optical density values on a microplate reader (Multiskan FC, Thermo Scientific, Braunschweig, Germany) at an absorbance of $450 \mathrm{~nm}$. 


\section{Animal experiments}

Animals were purchased from the Shanghai Experimental Animal Center, Chinese Academy of Science and housed under aseptic conditions at Zhejiang Academy of Medical Sciences. All procedures and maintenance conditions were approved by the Ethics Committee of the First Affiliated Hospital, Zhejiang University School of Medicine.

\section{Maximum tolerated dose (MTD) studies in normal mice}

The MTDs of free CTX and CTX prodrug-assembled micelles were investigated in healthy female ICR mice (4-5 weeks old) using a dose-escalation study. The animals were randomized into seven groups (n =3). Mice were intravenously (i.v.) injected with physiological saline, CTX in Jevtana-mimicking formulation $(10,15$, and $20 \mathrm{mg} / \mathrm{kg}$ ), or cabazitaxel-NPs $(40,50$, and $60 \mathrm{mg} / \mathrm{kg}$, CTX-equivalent) three times on days 0,3 , and 6 . Mouse survival and variation in body weight were monitored every three days.

The MTD was defined as the maximum dose level allowing a temporary body weight loss within $20 \%$ and without causing any death-related incidents throughout the administration.

\section{Evaluation of in vivo drug toxicity}

To assess the safety profile of MK-2206 in combination with CTX of distinct formulations, healthy ICR mice were treated with free CTX (6 mg/ kg, 60\% MTD, i.v.) plus MK-2206 (50 mg/kg, i.p.) and cabazitaxelNPs (24 mg/kg, 60\% MTD, i.v.) plus MK-2206 (50 mg/kg, i.p.) thrice (on days 0, 3 and 6). Mice that received saline administration were included as controls. One day post-final administration, the serum of each treated mouse was collected to examine the biochemical indexes of hepatic and renal function (aspartate transaminase, AST; alanine aminotransferase, ALT; alkaline phosphatase, ALP; blood urea nitrogen, BUN; serum creatinine, Scr). The major organs of mice were also excised for hematoxylin-eosin (H\&E) staining analysis. For the myelosuppression study, the blood samples from each treatment group were collected, and peripheral blood parameters were measured. The values are expressed as the means $\pm \operatorname{SD}(n=6)$.

\section{Therapeutic efficacy in a HeLa/Taxol xenograft model and histological analysis}

To produce tumors, HeLa/Taxol cells $\left(7 \times 10^{5}\right.$ cells) were subcutaneously injected into the right flank of female athymic BALB/c nude mice ( $4-6$ weeks old, $n=6$ ). When the tumor sizes reached $\sim 100 \mathrm{~mm}^{3}$, tumor-bearing mice were randomized into six groups as follows: i) saline; ii) MK-2206 (25 mg/kg, i.p.); iii) clinically formulated CTX (3 mg/kg, i.v.); iv) cabazitaxel-NPs ( $12 \mathrm{mg} / \mathrm{kg}$ at CTX-equivalence, i.v.); v) MK2206 plus CTX; vi) MK-2206 plus cabazitaxel-NPs. Medical interventions were given at days 0, 3, and 6 . The mice were continuously monitored every second day by recording tumor volumes as well as body weights. All mice were sacrificed when mice in the control group reached humane endpoints, and the tumors were weighed and photographed immediately. The tumor inhibition rate was calculated using the 
following equation: inhibition rate $(\%)=\left(W_{c}-W_{t}\right) / W_{c} \times 100 \%$, where $W_{c}$ and $W_{t}$ represented the average tumor weight of saline- and drug-treated groups, respectively.

Subsequent histological analyses were performed on tumor tissues treated with the above-mentioned regimens. Briefly, tumor tissues were sequentially fixed with $4 \%$ paraformaldehyde, embedded in paraffin, serially sectioned, and stained with hematoxylin-eosin. To assess inhibition of tumor proliferative capacity as well as induction of cancer cell apoptosis, tumor sections were immunohistologically stained with Ki-67 and deoxynucleotidyl transferase-mediated dUTP nick end labeling (TUNEL), respectively.

\section{Statistical analysis}

All of the data are expressed as the means \pm standard deviation (SD) of three independent measurements. Statistical comparisons between two groups were made using Student's t-test, where statistical significance was assigned for a $P$ value of $<0.05$. Comparisons between multiple groups were made with one-way parametric analysis of variance (ANOVA).

\section{Results}

\section{Preparation and characterization of cabazitaxel conjugate- loaded nanoparticles}

The oligolactide-tethered cabazitaxel conjugate (termed OLA-CTX) was synthesized according to our previous protocol [22]. Further incorporation of the OLA-CTX conjugate into micelles composed of the $\mathrm{PEG}_{5 \mathrm{k}}-b-P L A_{8 \mathrm{k}}$ matrix enabled the formation of systemically injectable nanoparticles (termed cabazitaxelNPs, Fig. 1a). As revealed by transmission electron microscopy (TEM) observations, the NPs were spherical in shape, and the mean diameter of the NPs in the TEM images was $26.2 \pm 9.2 \mathrm{~nm}$ (Fig. 1b). The size distribution of cabazitaxel-NPs was also characterized by dynamic light scattering (DLS). As shown in Fig. 1c, cabazitaxel-NPs exhibited a narrow size distribution, with a low polydispersity index (PDI = $0.118 \pm 0.009)$ and a median hydrodynamic diameter $\left(D_{\mathrm{H}}\right)$ of $\sim 57 \mathrm{~nm}$. In addition, these NPs had improved thermodynamic stability, with neither an apparent change in particle size nor macroscopic precipitation in PBS- or $20 \%$ FBS-containing solutions when stored at $4{ }^{\circ} \mathrm{C}$ over 2 weeks (Fig. 1 d). Thus, chemical derivatization of the parent cabazitaxel drug with an oligolactide motif increases the compatibility and miscibility with the $\mathrm{PEG}_{5 \mathrm{k}}-b-\mathrm{PLA}_{8 \mathrm{k}}$ matrices, rendering the NPs injectable and circulatory for long periods.

\section{MK2206 reverses Akt activation induced by cabazitaxel treatment}

To determine whether cabazitaxel could effectively kill paclitaxel-resistant cancer cells, we examined the growth inhibitory effect by comparing the $\mathrm{IC}_{50}$ values between paclitaxel-resistant cancer cells (i.e., A549/Taxol and HeLa/Taxol) and their parental cells (i.e., A549 and HeLa). As shown in Fig. 2a and c and 
summarized in Table 1, both HeLa/Taxol and A549/Taxol cells exhibited resistance to paclitaxel, as evidenced by 15.6 -fold and 140.4 -fold higher $\mathrm{IC}_{50}$ values than their corresponding naïve cells, respectively. However, treatment with cabazitaxel dramatically decreased the $\mathrm{IC}_{50}$ values for these two resistant cell lines, indicating that cabazitaxel has the ability to partially, but not fully, overcome drug resistance, which is consistent with its reported low affinity toward P-gp (Fig. 2b, d).

Table 1

Effect of MK-2206 on reversing MDR in two paclitaxel-resistant cell lines.

\begin{tabular}{|c|c|c|c|c|}
\hline \multirow[t]{2}{*}{ Compounds } & \multicolumn{4}{|c|}{$\begin{array}{l}\mathrm{IC}_{50} \pm \text { Std (nM) } \\
\text { (fold-reversal) }\end{array}$} \\
\hline & HeLa & HeLa/Taxol & A549 & A549/Taxol \\
\hline Paclitaxel & $8.58 \pm 1.29$ & $133.60 \pm 7.90$ & $12.53 \pm 0.78$ & $1759.00 \pm 61.28$ \\
\hline Cabazitaxel & $\begin{array}{l}10.65 \pm 1.16 \\
(1.00)\end{array}$ & $\begin{array}{l}19.74 \pm 1.05 \\
(1.00)\end{array}$ & $\begin{array}{l}5.18 \pm 0.39 \\
(1.00)\end{array}$ & $\begin{array}{l}22.14 \pm 1.99 \\
(1.00)\end{array}$ \\
\hline$+0.33 \mu \mathrm{M}$ MK-2206 & $\begin{array}{l}5.84 \pm 0.51 \\
(1.82)\end{array}$ & $\begin{array}{l}15.67 \pm 0.76 \\
(1.26)\end{array}$ & $\begin{array}{l}4.00 \pm 0.30 \\
(1.30)\end{array}$ & $\begin{array}{l}17.68 \pm 1.67 \\
(1.25)\end{array}$ \\
\hline$+1.00 \mu \mathrm{M}$ MK-2206 & $\begin{array}{l}4.55 \pm 0.30 \\
(2.34)\end{array}$ & $\begin{array}{l}9.01 \pm 0.90 \\
(2.19)\end{array}$ & $\begin{array}{l}3.71 \pm 0.22 \\
(1.40)\end{array}$ & $\begin{array}{l}10.05 \pm 0.96 \\
(2.20)\end{array}$ \\
\hline$+3.00 \mu \mathrm{M}$ MK-2206 & $\begin{array}{l}3.57 \pm 0.24 \\
(2.98)\end{array}$ & $\begin{array}{l}5.65 \pm 0.57 \\
(3.50)\end{array}$ & $\begin{array}{l}3.25 \pm 0.18 \\
(1.60)\end{array}$ & $\begin{array}{l}5.54 \pm 0.57 \\
(4.00)\end{array}$ \\
\hline Cabazitaxel-NPs & $\begin{array}{l}40.29 \pm 6.29 \\
(1.00)\end{array}$ & $\begin{array}{l}80.31 \pm 7.74 \\
(1.00)\end{array}$ & $\begin{array}{l}136.80 \pm 2.80 \\
(1.00)\end{array}$ & $\begin{array}{l}166.20 \pm 26.48 \\
(1.00)\end{array}$ \\
\hline$+0.33 \mu \mathrm{M}$ MK-2206 & $\begin{array}{l}17.00 \pm 2.43 \\
(2.37)\end{array}$ & $\begin{array}{l}61.16 \pm 5.61 \\
(1.31)\end{array}$ & $\begin{array}{l}105.10 \pm 11.80 \\
(1.30)\end{array}$ & $\begin{array}{l}119.40 \pm 20.12 \\
(1.39)\end{array}$ \\
\hline$+1.00 \mu \mathrm{M}$ MK-2206 & $\begin{array}{l}10.28 \pm 1.38 \\
(3.92)\end{array}$ & $\begin{array}{l}43.20 \pm 4.27 \\
(1.86)\end{array}$ & $\begin{array}{l}78.03 \pm 8.24 \\
(1.75)\end{array}$ & $\begin{array}{l}65.61 \pm 11.70 \\
(2.53)\end{array}$ \\
\hline$+3.00 \mu \mathrm{M}$ MK-2206 & $\begin{array}{l}8.40 \pm 1.08 \\
(4.80)\end{array}$ & $\begin{array}{l}27.53 \pm 3.38 \\
(2.92)\end{array}$ & $\begin{array}{l}61.29 \pm 6.54 \\
(2.23)\end{array}$ & $\begin{array}{l}23.25 \pm 7.44 \\
(7.15)\end{array}$ \\
\hline
\end{tabular}


To potentiate the cytotoxic activity of cabazitaxel in these resistant cells, we included the pan-Akt inhibitor MK-2206 as a chemosensitizer for efficacy testing. Akt has been identified as a master regulator of hundreds of downstream substrates involved in cell metabolism, growth, survival, and proliferation. More importantly, the Akt signaling pathway is also associated with chemotherapy resistance. Prior studies have shown that exposure to paclitaxel treatment aberrantly activates Akt via phosphorylation, thereby preventing cancer cells from undergoing apoptosis and producing drug resistance [20]. In addition, deactivation of the Akt pathway with small-molecule inhibitors has been demonstrated to sensitize resistant cancer cells to chemotherapeutic agents [23]. Therefore, we suspected that cabazitaxel would trigger an increase of activated Akt in cancer cells as a protection mechanism, and Akt inhibition would render cells vulnerable to cabazitaxel poisons. Ultimately, Akt inhibition was expected to generate a better synergistic effect on taxane-resistant cells.

To validate this hypothesis, we first treated HeLa/Taxol and A549/Taxol cells with $10 \mathrm{nM}$ cabazitaxel for different times. Strikingly, the addition of cabazitaxel significantly induced the activation of Akt in both cell lines in a time-dependent manner (Fig. 2e, f). Meanwhile, MK-2206 significantly inhibited Akt phosphorylation at a wide range of concentrations (Fig. 2g, h). Coincubation with MK-2206 completely reversed cabazitaxel-induced Akt activation (Fig. 2i, j). These results suggest that targeting the Akt pathway may potentially be useful to improve the activity of cabazitaxel agents in taxane-resistant cancer cells. To prove this, we further examined cell viability in the presence of an Akt inhibitor, MK-2206. The $\mathrm{IC}_{50}$ values of cabazitaxel combined with MK-2206 are summarized in Table 1 . The addition of noncytotoxic concentrations of MK-2206 reversed the MDR-mediated resistance and produced a greater reversal effect in resistant cells than in sensitive cells, showing fold reversal values. Further examination of the in vitro cytotoxicity showed that OLA-CTX-loaded NP preserved the combinatorial efficacy at reducing cell viability with MK-2206, although NP was less potent than free cabazitaxel against both sensitive and resistant cells, possibly a result of sustained release and cleavage of ester linkages to generate active drug from the NP (Table 1). These results provide compelling evidence that MK-2206 potentiates the efficacy of free or particulate cabazitaxel in cancer cells.

\section{Combination of the Akt inhibitor with nanotherapy induces enhanced antiproliferative and procytotoxic activities in resistant cells}

To explore the combinatorial anti-proliferative activity in resistant cells, we performed a 5-ethynyl-2'deoxyuridine (EdU) assay, which enables specific measurement of DNA replication (Fig. 3a, b). In accordance with the results obtained in the CCK-8 assay, MK-2206 showed negligible anti-proliferation activity in two resistant cell lines. Cabazitaxel-NPs significantly inhibited cell proliferation, and the efficacy was augmented by the combination of MK-2206. For instance, the relative proliferation rate exhibited a 2.38-fold decrease after NP treatment in HeLa/Taxol cells, and further combined treatment with MK-2206 substantially reduced the rate by 5.16 -fold. Meanwhile, we conducted a colony formation assay to show the synergy of the combined treatment. Two resistant cell lines were incubated with increasing concentrations of both therapeutics for 2 weeks (Fig. 3c). As expected, MK-2206 had a negligible impact on cell growth but significantly inhibited cell colony formation when combined with 
varying concentrations of cabazitaxel-NPs. In particular, $1 \mu \mathrm{mol} / \mathrm{L}$ MK-2206 markedly enhanced NPmediated suppression of colony formation when the concentration of the latter reached $5 \mathrm{nM}$ in HeLa/Taxol cells and $25 \mathrm{nM}$ in A549/Taxol cells. These results indicated that combining MK-2206 with NPs synergistically inhibited cell proliferation and colony formation against paclitaxel-resistant tumor cells.

To investigate whether the collaborative suppression of cell proliferation was accompanied by enhanced induction of apoptosis, we performed annexin V/propidium iodide $(\mathrm{PI})$ double staining to evaluate apoptosis. In the flow cytometric analysis, cells treated with MK-2206 alone did not show any discrepancy with untreated cells in apoptotic rates (Fig. 3d, e). In contrast, both cell lines exhibited an increased percentage of apoptotic cells when exposed to cabazitaxel-NPs. The apoptotic rate was further promoted by the combined treatment, increasing from $16.2-28.1 \%$ for HeLa/Taxol cells and from 14.728.4\% for A549/Taxol cells compared to single NP treatment. Western blot analysis indeed validated caspase-associated apoptosis in cells (Fig. 3f, g). The combined treatment markedly increased the expression levels of cleaved caspase-3 and cleaved PARP; however, neither MK-2206 alone nor low concentrations of NPs increased the levels of apoptosis proteins in either cell line. These data indicate that MK-2206 potentiates the efficacy of nanotherapy in inducing apoptotic events in resistant cells.

\section{Combined treatment of NP with MK-2206 synergistically arrests cells at the $\mathrm{G} 2 / \mathrm{M}$ phase}

To better understand the anticancer mechanism responsible for the synergistic activity, we analyzed the cell cycle distribution after drug treatment. Figure $4 a$ and $b$ show that, unlike other treatments, the addition of cabazitaxel-NPs with MK-2206 reached maximal G2/M phase arrest and the fewest cell proportion in the G0/G1 phase. Cabazitaxel primarily binds to the interface of microtubules and stabilizes them through inhibition of the disassembly of tubulin heterodimers. Importantly, microtubules are indispensable elements for the spindle apparatus, which plays a critical role in mitotic chromosome movements. Hence, these results are consistent with the mode of action of cabazitaxel, indicating the release of active compounds in their native form.

Disruption of mitotic spindle organization inevitably affects nuclear integrity. We therefore used Hoechst staining to visualize changes in cell nuclei. As presented in Fig. 4c, d (upper), no aberrant nuclei were observed in cells either treated with MK-2206 or without treatment. However, segregating nuclei emerged after the cells were exposed to cabazitaxel-NPs for $48 \mathrm{~h}$. Noticeably, scattered pyknotic nuclei (indicative of cell death) were observed within HeLa/Taxol cells, and the fraction of cells with defective mitoses was higher than that of A549/Taxol cells under the same conditions. Moreover, when cotreated with MK-2206, the number of multinucleated cells increased further in both cell lines, demonstrating that an extension of mitotic catastrophe was induced by additional Akt inhibition (Fig. 4e).

Considering that microtubules are basic components of the cytoskeletal array, cells were further costained with fluorescently labeled phalloidin to visualize the cellular architecture (Fig. 4c, d, middle). In parallel with the changes in the nucleus, a distinct increase in cellular volume was revealed in all 
cabazitaxel-NP-poisoned (either alone or combined) cells over that in MK-2206-treated cells as well as controls, indicating that the irregular transformation of cell morphology was synchronized with the aberrance in mitosis. The results were further validated by FCAS analysis (Fig. 4f), where the Y-axis represented a direct correlation with cell diameter. Altogether, these data support the hypothesis that MK2206 fosters the anticancer capability of cabazitaxel-NPs via defective mitoses and disrupted cellular architecture.

\section{MK-2206 potentiates the anti-metastatic effect of cabazitaxel-NPs}

In addition to the maintenance of cellular architecture, microtubule dynamics are also known to collaborate intimately with the actin cytoskeleton to orchestrate cell motility and adhesion [24,25]. For cancer cells, dysfunction of microtubule dynamics potentially affects their migratory and invasive abilities, both of which contribute to the metastatic cascade [26]. On this account, we performed in vitro anti-metastasis assays to interrogate the potential impact of the combined regimen on the metastatic features of resistant cells using subtoxic concentrations. Initially, the migration of the two resistant cell lines was investigated by a wound-healing assay. Figure $5 \mathrm{a}, \mathrm{b}$ displayed the wound closures after $36 \mathrm{~h}$ of treatment. Compared with the control group, monotherapy with MK-2206 slightly inhibited cell migration in both cell lines. In contrast, cabazitaxel-NPs markedly weakened the cell migratory activities, as the wound closure was lessened to $11.89 \%$ in HeLa/Taxol cells and $30.51 \%$ in A549/Taxol cells. Furthermore, the combined therapy restrained the migration capability in both resistant cell lines by more than $50 \%$ compared to the untreated control (Fig. 5c, d).

To evaluate the anti-invasive efficacy of the combined therapy, Matrigel-coated chambers were applied to imitate the pathological course of cancer invasion of the surrounding tissues. As depicted in Fig. 5 e, the invaded cells underneath the polycarbonate membrane were stained and randomly counted after $36 \mathrm{~h}$. Similar to the trends observed in the wound-healing assay, the combined therapy was highly potent at disrupting the invasive capabilities of the two resistant cell lines, as the number of invaded cells declined dramatically compared with untreated cells or cells with individual treatment. MK-2206 also suppressed the invasiveness of HeLa/Taxol cells and A549/Taxol cells to some degree, and the difference was statistically significant compared to the control (Fig. 5f).

We further explored the underlying mechanism of the combination therapy on cell metastasis via Western blotting. It is widely acknowledged that downregulation of E-cadherin with concomitant upregulation of $\mathrm{N}$-cadherin is associated with a more invasive phenotype of tumor cells, both of which serve as major hallmarks of the epithelial-to-mesenchymal transition (EMT), a compelling paradigm for studying cancer metastasis [27]. In this regard, we examined their expression levels in both HeLa/Taxol and A549/Taxol cells with various treatments. Upon combined treatment, a considerable increase in E-cadherin expression and a concomitant decrease in $\mathrm{N}$-cadherin expression were noticed, indicating reduced invasive capabilities of these resistant cells (Fig. $5 \mathrm{~g}, \mathrm{~h}$ ). In summary, these data provide strong evidence that cotreatment of cabazitaxel-NPs and MK-2206 subjugates the metastatic potential of the resistant 
cancers via modulation of EMT markers and, probably, disruption of dynamic microtubule-based cell motility.

\section{The combination of MK-2206 with cabazitaxel-NPs elicits novel actions in taxane-resistant cells}

The ATP-dependent membrane transporter P-gp plays a central role in drug expulsion and resistance to taxane drugs [28]. Although cabazitaxel is known to show marginal susceptibility to P-gp, its countereffect on P-gp remains elusive albeit attractive. We therefore examined the potential impact of cabazitaxel on P-gp expression. Fig. S4 first confirmed the overexpression of P-gp protein in the resistant cells compared to their naïve cells. Both of the resistant cells were then treated with paclitaxel or cabazitaxel at varying $\mathrm{IC}_{50}$ concentrations for $48 \mathrm{~h}$. Unexpectedly, a significant reduction in P-gp expression was observed after cabazitaxel treatment (Fig. 6a); however, no alteration of P-gp expression was validated in paclitaxel-treated cells. More interestingly, the addition of MK-2206 into cabazitaxel- or NP-treated cells further reduced the expression of P-gp (Fig. 6b, c). Nonetheless, P-gp expression was reversed by cabazitaxel treatment or combined with MK-2206, thereby highlighting a potential solution for overcoming drug resistance using cabazitaxel-based formulations.

We further attempted to gain insights into how Akt inhibition strengthens the potency of cabazitaxel nanomedicine. Akt inhibition is thought to facilitate the stabilization of microtubules, as the Akt pathway is involved in the regulation of microtubule dynamics [29]. Therefore, we assessed the expression level of acetylated a-tubulin, a marker of microtubule stability, in resistant cells [30]. Western blot analysis showed that NP treatment substantially increased the level of acetylated a-tubulin, which was further augmented when MK-2206 was added, indicating a synergistic effect on stabilizing microtubules (Fig. 6d, f). These findings were further confirmed by immunofluorescence studies, through which an increased red fluorescence signal (acetylated a-tubulin) was observed around the aberrant nuclei in cells upon combinatorial treatment (Fig. 6f). In contrast, NPs or MK-2206 alone produced low acetylation levels.

To validate that the stabilized microtubule was indeed a result of promoting tubulin polymerization, we further performed immunofluorescence staining to observe the formation of microtubule bundles, which are extremely stable and represent taxane activity [31]. Fluorescence microscopy imaging revealed that, after treatment with NPs or in combination with MK2206, microtubule bundles were clearly induced (Fig. 6g, arrowheads). In contrast, the microtubules in untreated or MK-2206-treated cells were regularly distributed throughout the cells. Taken together, these results suggest that MK-2206 renders resistant cells more susceptible to cabazitaxel-NP treatment by enhancing cabazitaxel-mediated microtubule stabilization. This eventually results in a reinforced mitotic catastrophe and cell apoptosis.

\section{In vivo safety profiles of cabazitaxel-NPs}

The use of cabazitaxel in the clinic has been greatly hampered by its severe side effects. Thus, a favorable therapeutic effect with reduced damage to healthy tissues is necessary for this potent agent. Here, we investigated whether the cabazitaxel prodrug-encapsulated platform could mitigate the in vivo safety concerns. For this purpose, we first examined the maximum tolerated dose (MTD) of the 
nanomedicine in healthy ICR mice, which can offer a gross overview of drug safety profiles [32]. High doses of cabazitaxel-NPs were tested at 40,50 , and $60 \mathrm{mg} / \mathrm{kg}$ (cabazitaxel-equivalent) on days 0,3 , and 6 via intravenous administration. Clinically formulated cabazitaxel was also intravenously injected into mice at doses of 10,15 , and $20 \mathrm{mg} / \mathrm{kg}$ with the same schedule. The healthy condition of mice was monitored over 2 weeks, and the loss of body weight greater than $20 \%$ or death was regarded as the humane endpoint (Fig. 7a, b). Unfortunately, the mice only tolerated free cabazitaxel at a dose of $10 \mathrm{mg} / \mathrm{kg}$, and dosing of cabazitaxel at 15 and $20 \mathrm{mg} / \mathrm{kg}$ reduced the body weight by more than $20 \%$ or caused substantial mouse death. A high dose of cabazitaxel-NPs at $40 \mathrm{mg} / \mathrm{kg}$ resulted in body weight loss, but it gradually rebounded to the normal range after cessation of injection. We thus conclude that the MTD value for cabazitaxel-NPs is 4 times higher than that of clinical cabazitaxel injection.

We next assessed whether cotherapy with MK-2206 had an impact on in vivo safety. Acute and chronic toxicities in healthy ICR mice after treatment with combined regimens were examined. Mice with $60 \%$ MTDs of cabazitaxel and cabazitaxel-NPs as well as $50 \mathrm{mg} / \mathrm{kg}$ MK-2206 were chosen and evaluated in vivo through tissue toxicology, hematology, and serology assessments. Fresh sera and primary organs of each group were collected one day post-final administration to determine acute toxic effects. Indicators of hepatic and renal function did not show any significant abnormalities in any group (Fig. 7c-g). Similarly, microscopic observation of tissue sections confirmed that there were no noticeable pathological changes in either group with combination treatments (Fig. 7h).

Furthermore, a complete panel of hematology was monitored by measurement of blood samples collected at every dose and at 1, 2, and 3 weeks after administration of the final dose. As summarized in Table S2, neutropenia accompanied by leukopenia was observed in both treatment groups at dose 2 . This situation was attenuated as the leukocyte counts began to rebound at dose 3 , and the neutropenia recovered one week after the final administration in both groups, indicating that these transient changes were manageable. Concurrently, all the remaining hematology parameters fluctuated within normal limits. Collectively, these results confirmed that combination regimens of MK-2206 with cabazitaxel (regardless of the formulation) at the indicated dosages did not increase the incidence of toxic side effects. Together, our nanotherapy of cabazitaxel had significantly higher tolerability than its commercial formulation. More specifically, the increased dosage of cabazitaxel nanomedicine was expected to be superior at surmounting paclitaxel resistance without causing adverse effects when administered in vivo, and such potency would be fully exploited with the assistance of MK-2206.

\section{In vivo antitumor studies of cabazitaxel-NPs in combination with MK-2206}

Encouraged by the excellent in vitro synergy as well as improved tolerance of the nanomedicine, we finally evaluated the efficacy of the combinatorial therapy in a preclinical mouse model of a paclitaxelresistant HeLa/Taxol xenograft. Considering that nude mice are less tolerant than ICR mice, half doses of those used in the in vivo safety evaluation were employed for this study. When the tumor volume reached $\sim 100 \mathrm{~mm}^{3}$, we initiated the treatment by administration of saline, MK-2206 $(25 \mathrm{mg} / \mathrm{kg}$ ), cabazitaxel (in Jevtana-mimicking formulation, $3 \mathrm{mg} / \mathrm{kg}$ ), cabazitaxel-NPs (12 mg/kg cabazitaxel-equiv. dose), or their 
combinations on days 0,3 and 6 . Rapid tumor growth was observed for this taxane-resistant cervical cancer when xenografted into female nude mice (Fig. 8a). Administration of cabazitaxel-NPs produced considerable tumor suppression, whereas free cabazitaxel at a tolerable dose (e.g., $3 \mathrm{mg} / \mathrm{kg}$ ) in the Jevtana-mimicking formulation negligibly retarded tumor growth. Impressively, the in vivo potency of cabazitaxel-NPs was further strengthened by co-administering MK-2206, in accordance with the results observed in cell-based experiments. The tumors in each group were further excised at the endpoint of the study and weighed to calculate tumor inhibition rates (Fig. 8c-e). The increased dosage of cabazitaxelNPs yielded extraordinary tumor growth inhibition (up to 80\%) when combined with Akt inhibition. The low toxicity of the nanoparticle treatment and combination therapy was supported by stable body weights in animals (Fig. 8b). A TUNEL assay of the excised HeLa/Taxol tumors revealed that efficient and extensive intratumoral apoptosis was induced by the concomitant administration of cabazitaxel-NPs and MK-2206, which correlated well with hematoxylin and eosin (H\&E) and the reduction in Ki-67 proliferation (Fig. 8f). Furthermore, intratumoral changes were also examined by Western blot analysis of tumor tissues (Fig. 8g). Consistent with the in vitro results, a reduction in P-gp expression was observed after NP treatment or a combinatorial regimen (Fig. 8g). Moreover, combinatorial therapy using MK-2206 and NP significantly increased the levels of cleaved PARP and acetyl-a-tubulin in tumors compared with either single-agent treatments or free drug combination. Thus, these data support the excellent in vivo antitumor efficacy of a de novo platform for nanodrug combination in surmounting tumor drug resistance.

\section{Discussion}

Taxane-based regimens, either used as monotherapy or in combination with other agents, have become the mainstay of treatment for a wide range of solid tumor types [33]. However, the Achilles hill of using taxanes (e.g., paclitaxel and docetaxel) emerges in the clinic because they unfortunately are substrates for multidrug resistance proteins [34]. Therefore, the development of novel antimicrotubule agents that overcome drug resistance is of high priority. Cabazitaxel is a newly developed semisynthetic taxane and has been proven to be more potent than first-generation taxanes in treating multidrug-resistant (MDR) cancer due to its reduced affinity for P-glycoprotein. Cabazitaxel was clinically approved in 2010 as a chemotherapeutic alternative for patients with hormone-refractory prostate cancer who relapsed after previous docetaxel treatment. However, the clinical use of cabazitaxel has been limited due to insufficient drug delivery and severe side effects, especially the occurrence of neutropenia and leukopenia in patients.

Currently, a variety of biocompatible nanosystems have been designed for the safe and efficient delivery of chemotherapeutics to tumor sites by exploiting the enhanced permeability and retention (EPR) effect and exemption from the mononuclear phagocyte system (MPS) [35]. Pharmaceutical approaches using PEG-b-PLA polymeric NPs are popularly used for this purpose [36-38]. Therefore, in this study, we sought to avoid the side effects of cabazitaxel and to improve in vivo tolerability by virtue of drug conjugation to improve the compatibility of the ester prodrugs with the PEG-b-PLA matrix. The resultant cabazitaxel-NPs exhibited a spherical structure with nanoscale size and were extremely stable. Considering that cabazitaxel monotherapy may not be sufficient to fully unleash its potential against paclitaxel-resistant cancers, we further used a combination regimen. Previous studies have shown that abnormal activation 
of Akt is engaged in the development of chemoresistance, likely due to the activation of Akt-associated pro-survival and anti-apoptosis pathways $[39,40]$. Inhibition of the Akt pathway was also proven to potentiate the activity of many chemotherapies, such as cisplatin, doxorubicin, and paclitaxel [41-43]. We thus were intrigued to examine whether cabazitaxel treatment mediated the activation of Akt signaling in resistant cells and whether further inhibition would augment therapeutics.

In the present study, we first confirmed that cabazitaxel induced an increase in Akt phosphorylation as a self-protection mechanism in resistant HeLa/Taxol and A549/Taxol cells. Phosphorylation of Akt was reversed by the addition of MK-2206, a novel allosteric inhibitor of Akt. The results were further supported by a cell viability assay. Moreover, the combinatorial treatment led to significantly higher activity in resistant cells than in their parental cells. Notably, MK-2206 alone at the tested concentrations did not produce cytotoxicity. More importantly, a sensitization effect of MK-2206 to cabazitaxel-NPs was observed, resulting in enhanced cell apoptosis. Morphological observation revealed that cells treated with combinatorial therapy showed extensive mitotic arrest since MK-2206 exacerbated the degree of aberrant nuclei and irregular cell structure caused by cabazitaxel-NPs.

Cabazitaxel is more potent than first-generation taxanes because of its lower affinity for P-gp. Similar results using cabazitaxel to combat paclitaxel-resistant cells were verified in our study. In addition, we interestingly found for the first time that the addition of cabazitaxel at relatively high concentrations reduced P-gp expression. This phenomenon was likely ascribable to the interruptions caused by cabazitaxel in the transcriptional activity as well as microtubule-mediated membrane trafficking of P-gp. In addition, further combined treatment significantly reduced the expression of P-gp in resistant cells in vitro and in vivo. These results expand our understanding of the clinical significance of cabazitaxel agents. To further gain insights into the mechanism responsible for the synergism, we investigated whether MK-2206 could enhance the microtubule-stabilizing function of cabazitaxel-NPs because of its involvement in microtubule dynamics. The increased amount of acetylated a-tubulin demonstrated that the inactivation of Akt by MK-2206 significantly enhanced the microtubule stabilization caused by cabazitaxel-NPs. Furthermore, immunolabeling of tubulin displayed an extensive array of microtubule bundles within the cells, a strong proof that the strengthened polymerization was induced by the combination treatment. These findings reveal that MK-2206 mediates the sensitivity of cabazitaxel-NPs by promoting microtubule stability, which in turn leads to a reinforced polymerizing effect of cabazitaxel as well as its capacity to trigger catastrophic segregation errors and reduce cancer cell viability.

The bulky polycyclic structure of cabazitaxel makes it a poorly water-soluble drug. Clinically, the formulation of cabazitaxel is assisted by cosolvents Tween-80 and ethanol, which unfortunately cause a series of severe side effects $[44,45]$. Therefore, the identification of optimal dosing regimens is important for the successful evaluation of cancer therapeutics, especially when therapies are combined. For this purpose, we first conducted a dose-escalation study to determine the MTDs of cabazitaxel both in the commercial formulation and our nanomedicine solution. The MTDs for cabazitaxel in the Jevtanamimicking system and nanoformulated cabazitaxel on a q036d schedule were $10 \mathrm{mg}$ cabazitaxel $/ \mathrm{kg}$ and $40 \mathrm{mg}$ cabazitaxel $/ \mathrm{kg}$, respectively. Further coadministration of their $60 \%$ MTDs with MK-2206 was 
proven to be biosafe in vivo according to a series of assessments. The results offer a gross overview of drug safety and can serve as a guideline for in vivo antitumor evaluation studies, where doses of $30 \%$ of their corresponding MTDs were used in combination with MK-2206. In the preclinical mouse model bearing paclitaxel-resistant HeLa/Taxol xenografts, cabazitaxel-NPs outperformed clinically formulated cabazitaxel in stunting tumor growth, and the boosted efficacy was a direct result of improved tolerability. Moreover, consistent with the results of monotherapies, there was prominently enhanced tumor growth inhibition in mice upon coadministration of cabazitaxel-NPs and MK-2206 compared with the other groups. The antitumor efficacy was further validated by tumor sections stained with H\&E, TUNEL, and Ki67, which showed that cotherapy with cabazitaxel nanodrug and MK-2206 elicited extensive apoptosis as well as reduced proliferation in tumors. In addition, no overt body weight loss was observed in each group of mice. Collectively, the data reported here clearly show that our nanomedicine-based strategy ascribes the improved biocompatibility to cabazitaxel, and the additional assistance of MK-2206 enables cabazitaxel-NPs to exert maximal efficiency against paclitaxel-resistant preclinical cancer models while sparing normal tissues.

\section{Conclusion}

Coadministration of cytotoxic cabazitaxel nanotherapeutics and a small-molecule inhibitor that targets Akt kinase was examined for the synergistic treatment of paclitaxel-resistant cancer. The prodrug strategy was leveraged to rationally engineer the potent and toxic cabazitaxel agent via conjugation with the oligolactide segment for formulation in PEG- $b$-PLA micelles. The resultant cabazitaxel-NPs featured reduced in vivo systemic toxicity while preserving their therapeutic efficacy. Activation of the Akt pathway induced by cabazitaxel was reversed by the Akt inhibitor MK-2206 in resistant cancer cells. Consequently, coadministration of MK-2206 along with cabazitaxel-NPs synergized to inhibit tumor growth in a preclinical mouse model bearing resistant xenografts. More interestingly, a high concentration of cabazitaxel was found to attenuate P-gp expression, although the mechanism requires further investigation. Collectively, these preclinical studies demonstrate the low-dose synergy of a cytotoxic drug with a molecular inhibitor, which can potentially provide great therapeutic benefits in curing patients with multidrug-resistant malignancies.

\section{Abbreviations}

P-gp: P-glycoprotein; MDR: multidrug resistance; PEG-b-PLA: poly(ethylene glycol)-block-poly (D,L-lactic acid); NP: nanoparticle; FDA: the US Food and Drug Administration; NSCLC: non-small cell lung cancer; CCK-8: cell counting Kit-8 assay; $\mathrm{IC}_{50}$ : 50\% inhibitory concentration; TEM: transmission electron microscopy; DLS: dynamic light scattering; PDI: polydispersity index; EdU: 5-Ethynyl-2'-deoxyuridine assay; MTD, maximum tolerated dose; AST: aspartate transaminase; ALT: alanine aminotransferase; ALP: alkaline phosphatase; BUN: blood urea nitrogen; Scr: serum creatinine; H\&E: Haematoxylin-eosin; TUNEL: deoxynucleotidyl transferase-mediated dUTP nick end labeling assay; EPR: enhanced permeability and retention; MPS: mononuclear phagocyte system; ANOVA: one-way parametric analysis of variance. 


\section{Declarations}

Acknowledgements

Not applicable.

\section{Authors' contributions}

WHX designed and supervised the study. LTY performed the experiment, analyzed the data, and drafted the manuscript. SLL, WJQ, and HXX conducted some experiments and collected the data. CWZ offered technical and material supports. All the listed authors read and approved the final manuscript.

\section{Funding}

This work was supported by grants from the Zhejiang Province Preeminence Youth Fund (grant LR19H160002) and the National Natural Science Foundation of China (grants 81773193 and 81571799 ).

\section{Availability of data and materials}

The datasets used and/or analyzed during the current study are available from the corresponding author on reasonable request.

\section{Ethics approval}

All procedures and maintenance conditions were approved by the Ethics Committee of the First Affiliated Hospital, Zhejiang University School of Medicine. All animal experiments were gently conducted to minimize animal pain and suffering. Animals were euthanized if the excessive deterioration of animal health was noted.

\section{Consent for publication}

All authors declare that they agree to publish the article.

\section{Competing interests}

The authors declare no potential conflicts of interest.

\section{Author details}

${ }^{1}$ The First Affiliated Hospital, Zhejiang University School of Medicine; NHC Key Laboratory of Combined Multi-Organ Transplantation; Key Laboratory of Organ Transplantation, Research Center for Diagnosis and Treatment of Hepatobiliary Diseases, Zhejiang Province, Hangzhou, 310003, PR China. ${ }^{2}$ Department of Medical Oncology; Sir Run Run Shaw Hospital; School of Medicine, Zhejiang University, Hangzhou, 310016, PR China. ${ }^{3}$ Department of Chemistry, Zhejiang University, Hangzhou, 310028, PR China.

\section{References}


1. Fitzpatrick JM, de Wit R. Taxane mechanisms of action: potential implications for treatment sequencing in metastatic castration-resistant prostate cancer. Eur Urol. 2014;65:1198-204.

2. Fojo T, Menefee M. Mechanisms of multidrug resistance: the potential role of microtubule-stabilizing agents. Ann Oncol. 2007;18(Suppl 5):v3-8.

3. Vrignaud $P$, Sémiond $D$, Lejeune $P$, Bouchard $H$, Calvet $L$, Combeau $C$, et al. Preclinical antitumor activity of cabazitaxel, a semisynthetic taxane active in taxane-resistant tumors. Clin Cancer Res. 2013;19:2973-83.

4. Barve A, Jain A, Liu H, Zhao Z, Cheng K. Enzyme-responsive polymeric micelles of cabazitaxel for prostate cancer targeted therapy. Acta Biomater. 2020;113:501-11.

5. Galsky MD, Dritselis A, Kirkpatrick P, Oh WK. Cabazitaxel. Nat Rev Drug Discov. 2010;9:677-8.

6. Sun B, Chitgupi U, Li C, Federizon J, Zhang C, Ruszaj D, et al. Surfactant-Stripped Cabazitaxel Micelles Stabilized by Clotrimazole or Mifepristone. Advanced Therapeutics. 2020;3:1900161.

7. Dorff TB, Quinn DI. Cabazitaxel in prostate cancer: stretching a string. Lancet. 2010;376:1119-20.

8. Sofias AM, Dunne M, Storm G, Allen C. The battle of "nano" paclitaxel. Adv Drug Deliv Rev. 2017;122:20-30.

9. Iqbal S, Blenner M, Alexander-Bryant A, Larsen J. Polymersomes for Therapeutic Delivery of Protein and Nucleic Acid Macromolecules: From Design to Therapeutic Applications. Biomacromol. 2020;21:1327-50.

10. Wang $H$, Zhou L, Xie K, Wu J, Song P, Xie H, et al. Polylactide-tethered prodrugs in polymeric nanoparticles as reliable nanomedicines for the efficient eradication of patient-derived hepatocellular carcinoma. Theranostics. 2018;8:3949-63.

11. Grossen P, Witzigmann D, Sieber S, Huwyler J. PEG-PCL-based nanomedicines: A biodegradable drug delivery system and its application. J Control Release. 2017;260:46-60.

12. Lee KS, Chung HC, Im SA, Park YH, Kim CS, Kim SB, et al. Multicenter phase II trial of Genexol-PM, a Cremophor-free, polymeric micelle formulation of paclitaxel, in patients with metastatic breast cancer. Breast Cancer Res Treat. 2008;108:241-50.

13. Ulbrich K, Holá K, Šubr V, Bakandritsos A, Tuček J, Zbořil R. Targeted Drug Delivery with Polymers and Magnetic Nanoparticles: Covalent and Noncovalent Approaches, Release Control, and Clinical Studies. Chem Rev. 2016;116:5338-431.

14. Doane T, Burda C. Nanoparticle mediated non-covalent drug delivery. Adv Drug Deliv Rev. 2013;65:607-21.

15. Song M, Bode AM, Dong Z, Lee MH. AKT as a Therapeutic Target for Cancer. Cancer Res. 2019;79:1019-31.

16. Revathidevi S, Munirajan AK. Akt in cancer: Mediator and more. Semin Cancer Biol. 2019;59:80-91.

17. Agarwal E, Chaudhuri A, Leiphrakpam PD, Haferbier KL, Brattain MG, Chowdhury S. Akt inhibitor MK2206 promotes anti-tumor activity and cell death by modulation of AIF and Ezrin in colorectal cancer. BMC Cancer. 2014;14:145. 
18. Li L, Wei XH, Pan YP, Li HC, Yang H, He QH, et al. LAPTM4B: a novel cancer-associated gene motivates multidrug resistance through efflux and activating PI3K/AKT signaling. Oncogene. 2010;29:5785-95.

19. VanderWeele DJ, Zhou R, Rudin CM. Akt up-regulation increases resistance to microtubule-directed chemotherapeutic agents through mammalian target of rapamycin. Mol Cancer Ther. 2004;3:160513.

20. Sun H, Yu T, Li J. Co-administration of perifosine with paclitaxel synergistically induces apoptosis in ovarian cancer cells: more than just AKT inhibition. Cancer Lett. 2011;310:118-28.

21. Li Z, Yan S, Attayan N, Ramalingam S, Thiele CJ. Combination of an allosteric Akt Inhibitor MK-2206 with etoposide or rapamycin enhances the antitumor growth effect in neuroblastoma. Clin Cancer Res. 2012;18:3603-15.

22. Wan J, Qiao Y, Chen X, Wu J, Zhou L, Zhang J, et al. Structure-Guided Engineering of Cytotoxic Cabazitaxel for an Adaptive Nanoparticle Formulation: Enhancing the Drug Safety and Therapeutic Efficacy. Adv Funct Mater. 2018;28:1804229.

23. Wu YH, Huang YF, Chen CC, Chou CY. Akt inhibitor SC66 promotes cell sensitivity to cisplatin in chemoresistant ovarian cancer cells through inhibition of COL11A1 expression. Cell Death Dis. 2019;10:322.

24. Zaoui K, Benseddik K, Daou P, Salaün D, Badache A. ErbB2 receptor controls microtubule capture by recruiting ACF7 to the plasma membrane of migrating cells. Proc Natl Acad Sci U S A. 2010;107:18517-22.

25. Etienne-Manneville S. Actin and microtubules in cell motility: which one is in control? Traffic. 2004;5:470-7.

26. Lupo B, Vialard J, Sassi F, Angibaud P, Puliafito A, Pupo E, et al. Tankyrase inhibition impairs directional migration and invasion of lung cancer cells by affecting microtubule dynamics and polarity signals. BMC Biol. 2016;14:5.

27. Serrano-Gomez SJ, Maziveyi M, Alahari SK. Regulation of epithelial-mesenchymal transition through epigenetic and post-translational modifications. Mol Cancer. 2016;15:18.

28. Mao C, Li F, Zhao Y, Debinski W, Ming X. P-glycoprotein-targeted photodynamic therapy boosts cancer nanomedicine by priming tumor microenvironment. Theranostics. 2018;8:6274-90.

29. Buttrick GJ, Wakefield JG. PI3-K and GSK-3: Akt-ing together with microtubules. Cell Cycle. 2008;7:2621-5.

30. Yu Y, Gaillard S, Phillip JM, Huang TC, Pinto SM, Tessarollo NG, et al. Inhibition of Spleen Tyrosine Kinase Potentiates Paclitaxel-Induced Cytotoxicity in Ovarian Cancer Cells by Stabilizing Microtubules. Cancer Cell. 2015;28:82-96.

31. Ahmed AA, Mills AD, Ibrahim AE, Temple J, Blenkiron C, Vias M, et al. The extracellular matrix protein TGFBI induces microtubule stabilization and sensitizes ovarian cancers to paclitaxel. Cancer Cell. 2007;12:514-27. 
32. Liu P, Situ JQ, Li WS, Shan CL, You J, Yuan H, et al. High tolerated paclitaxel nano-formulation delivered by poly (lactic-co-glycolic acid)-g-dextran micelles to efficient cancer therapy. Nanomedicine. 2015;11(4):855-66.

33. Ning N, Yu Y, Wu M, Zhang R, Zhang T, Zhu C, et al. A Novel Microtubule Inhibitor Overcomes Multidrug Resistance in Tumors. Cancer Res. 2018;78:5949-57.

34. Xie B, Wan J, Chen X, Han W, Wang H. Preclinical Evaluation of a Cabazitaxel Prodrug Using Nanoparticle Delivery for the Treatment of Taxane-Resistant Malignancies. Mol Cancer Ther. 2020;19:822-34.

35. Liu Y, Wang Z, Liu Y, Zhu G, Jacobson O, Fu X, et al. Suppressing Nanoparticle-Mononuclear Phagocyte System Interactions of Two-Dimensional Gold Nanorings for Improved Tumor Accumulation and Photothermal Ablation of Tumors. ACS Nano. 2017;11:10539-48.

36. Cao ZT, Gan LQ, Jiang W, Wang JL, Zhang HB, Zhang Y, et al. Protein Binding Affinity of Polymeric Nanoparticles as a Direct Indicator of Their Pharmacokinetics. ACS Nano. 2020;14:3563-75.

37. Xu C, Li D, Cao Z, Xiong M, Yang X, Wang J. Facile Hydrophobization of siRNA with Anticancer Drug for Non-Cationic Nanocarrier-Mediated Systemic Delivery. Nano Lett. 2019;19:2688-93.

38. Xu L, Xu S, Wang H, Zhang J, Chen Z, Pan L, et al. Enhancing the Efficacy and Safety of Doxorubicin against Hepatocellular Carcinoma through a Modular Assembly Approach: The Combination of Polymeric Prodrug Design, Nanoparticle Encapsulation, and Cancer Cell-Specific Drug Targeting. ACS Appl Mater Interfaces. 2018;10:3229-40.

39. Cioce M, Canino C, Goparaju C, Yang H, Carbone M, Pass HI. Autocrine CSF-1R signaling drives mesothelioma chemoresistance via AKT activation. Cell Death Dis. 2014;5:e1167.

40. Abedini MR, Muller EJ, Bergeron R, Gray DA, Tsang BK. Akt promotes chemoresistance in human ovarian cancer cells by modulating cisplatin-induced, p53-dependent ubiquitination of FLICE-like inhibitory protein. Oncogene. 2010;29:11-25.

41. Gohr K, Hamacher A, Engelke LH, Kassack MU. Inhibition of PI3K/Akt/mTOR overcomes cisplatin resistance in the triple negative breast cancer cell line HCC38. BMC Cancer. 2017;17:711.

42. Smolensky D, Rathore K, Bourn J, Cekanova M. Inhibition of the PI3K/AKT Pathway Sensitizes Oral Squamous Cell Carcinoma Cells to Anthracycline-Based Chemotherapy In Vitro. J Cell Biochem. 2017;118:2615-24.

43. Liu X, Xie C, Li A, Zhang Y, Liu X, Zhou S, et al. BEZ235 enhances chemosensitivity of paclitaxel in hepatocellular carcinoma through inhibiting the PI3K/Akt/mTOR pathway. Am J Transl Res. 2019;11:7255-71.

44. Wang H, Lu Z, Wang L, Guo T, Wu J, Wan J, et al. New Generation Nanomedicines Constructed from Self-Assembling Small-Molecule Prodrugs Alleviate Cancer Drug Toxicity. Cancer Res. 2017;77:6963-74.

45. Zhou L, Xie H, Chen X, Wan J, Xu S, Han Y, et al. Dimerization-induced self-assembly of a redoxresponsive prodrug into nanoparticles for improved therapeutic index. Acta Biomater. 2020;113:46477. 


\section{Figures}

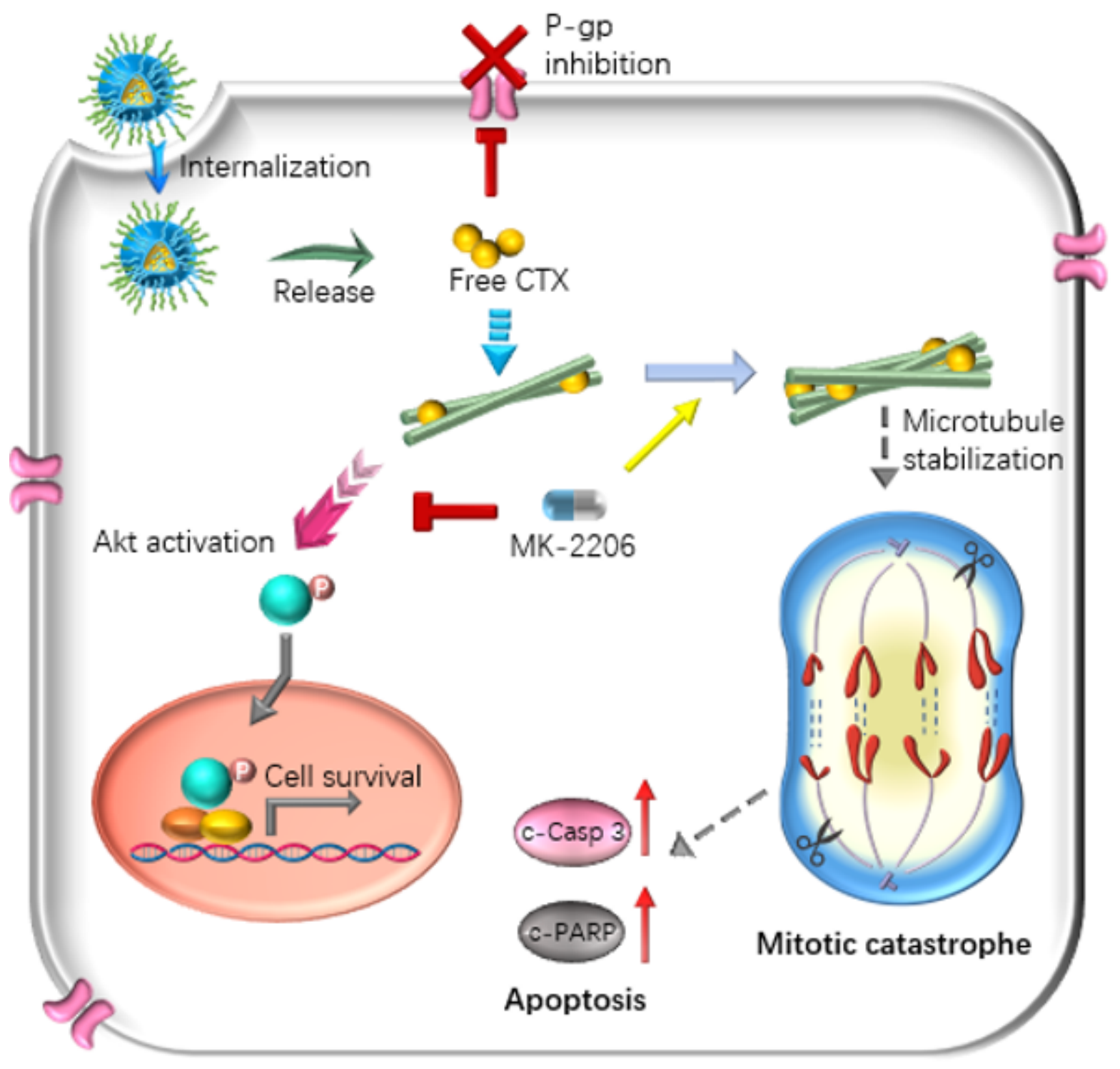

Figure 1

A concept schematic diagram elucidating how MK-2206 medication potentiates the effect of cabazitaxelNPs in resistant cancers. A CTX prodrug-based nanoplatform was readily prepared for intravenous administration by encapsulating the conjugates into amphiphilic copolymers. After circulation in blood vessels, the nanomedicines preferentially accumulate in tumors, and the active CTX is liberated postcellular internalization. CTX primarily exerts its cytotoxic effects by stabilizing the microtubules that are essential for cellular activities. However, the role of CTX is gradually counteracted by the cells as they adapt to activate the self-protective protein Akt. Nevertheless, this situation can be changed after the arrival of MK-2206 in the cytosol, an Akt inhibitor, which deactivates Akt and also augments the microtubule-stabilizing effect of CTX. With such a one-two punch, the resistant cancers are eventually eliminated. 


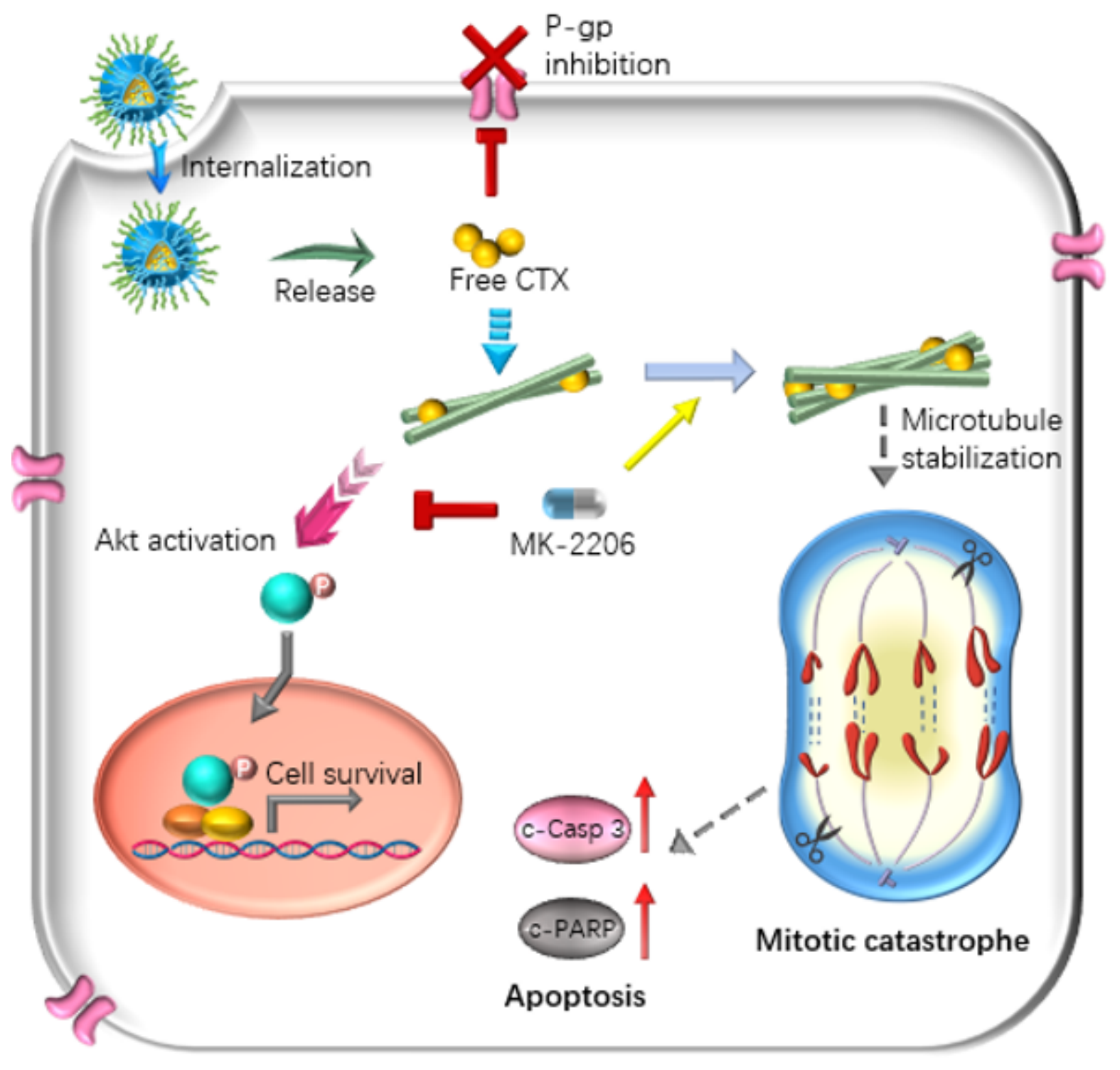

Figure 1

A concept schematic diagram elucidating how MK-2206 medication potentiates the effect of cabazitaxelNPs in resistant cancers. A CTX prodrug-based nanoplatform was readily prepared for intravenous administration by encapsulating the conjugates into amphiphilic copolymers. After circulation in blood vessels, the nanomedicines preferentially accumulate in tumors, and the active CTX is liberated postcellular internalization. CTX primarily exerts its cytotoxic effects by stabilizing the microtubules that are essential for cellular activities. However, the role of CTX is gradually counteracted by the cells as they adapt to activate the self-protective protein Akt. Nevertheless, this situation can be changed after the arrival of MK-2206 in the cytosol, an Akt inhibitor, which deactivates Akt and also augments the microtubule-stabilizing effect of CTX. With such a one-two punch, the resistant cancers are eventually eliminated. 


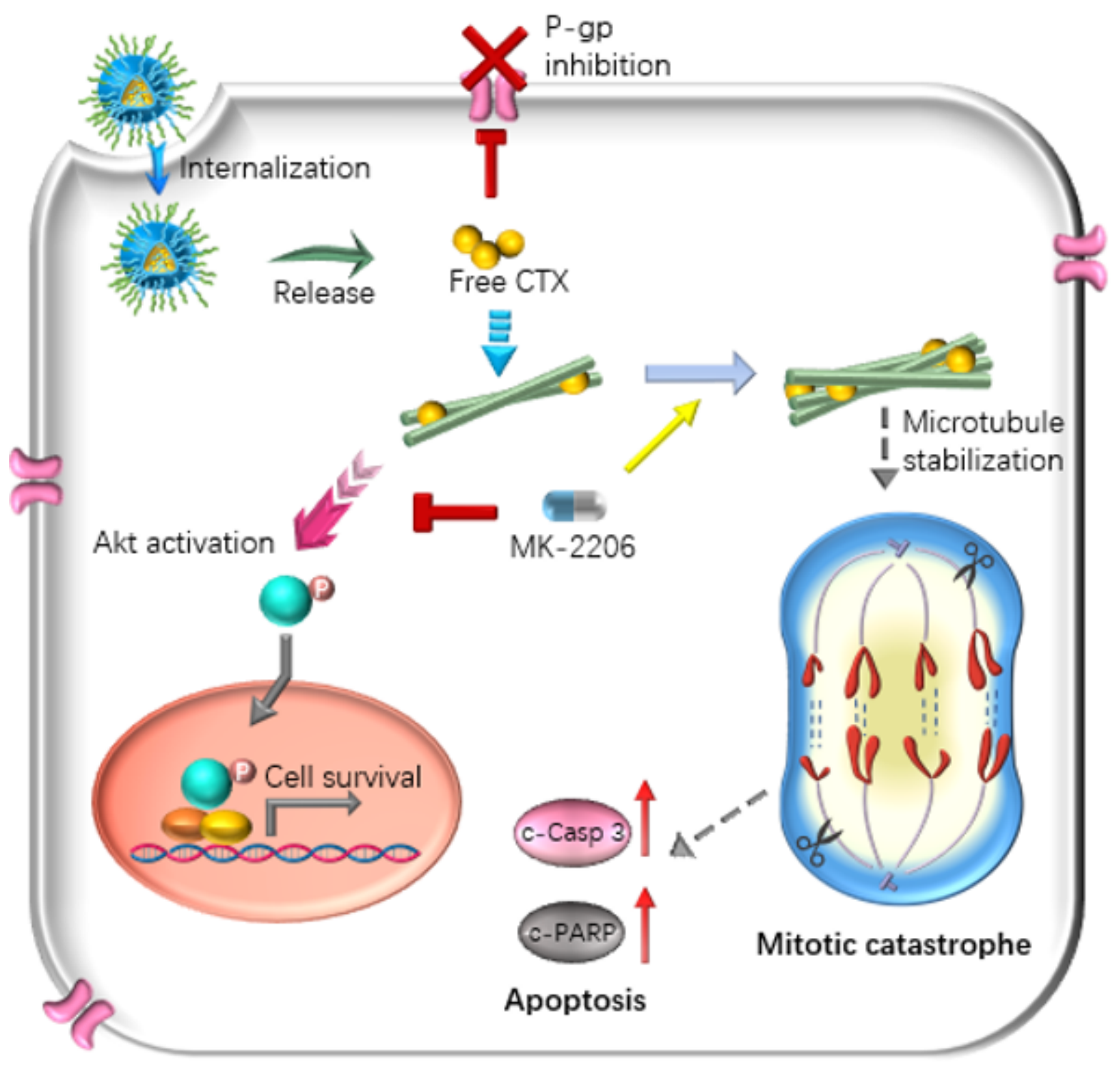

\section{Figure 1}

A concept schematic diagram elucidating how MK-2206 medication potentiates the effect of cabazitaxelNPs in resistant cancers. A CTX prodrug-based nanoplatform was readily prepared for intravenous administration by encapsulating the conjugates into amphiphilic copolymers. After circulation in blood vessels, the nanomedicines preferentially accumulate in tumors, and the active CTX is liberated postcellular internalization. CTX primarily exerts its cytotoxic effects by stabilizing the microtubules that are essential for cellular activities. However, the role of CTX is gradually counteracted by the cells as they adapt to activate the self-protective protein Akt. Nevertheless, this situation can be changed after the arrival of MK-2206 in the cytosol, an Akt inhibitor, which deactivates Akt and also augments the microtubule-stabilizing effect of CTX. With such a one-two punch, the resistant cancers are eventually eliminated. 
A

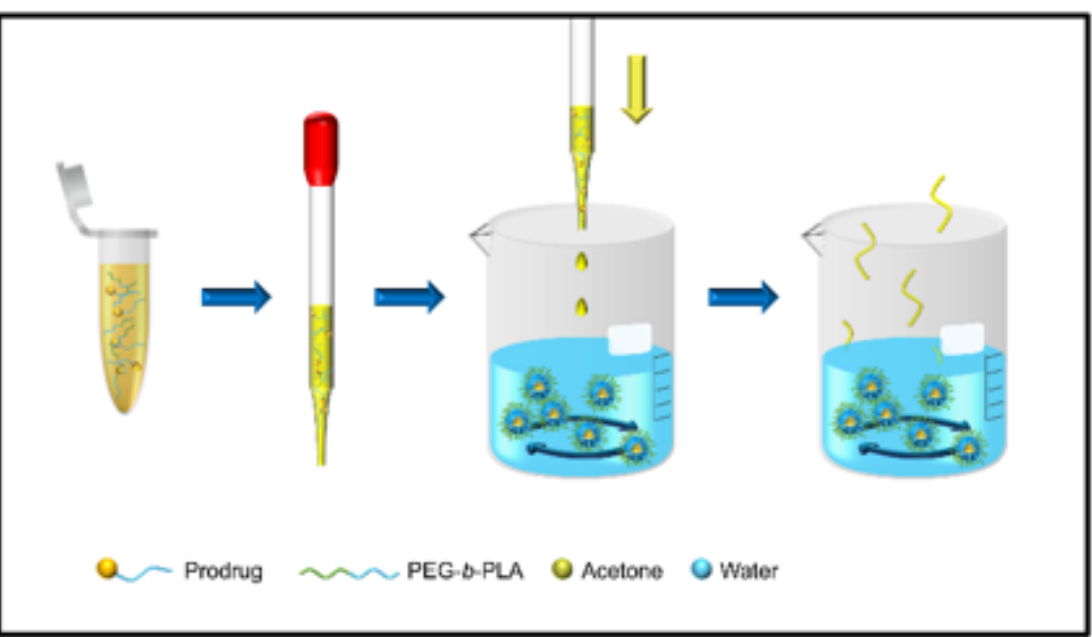

B
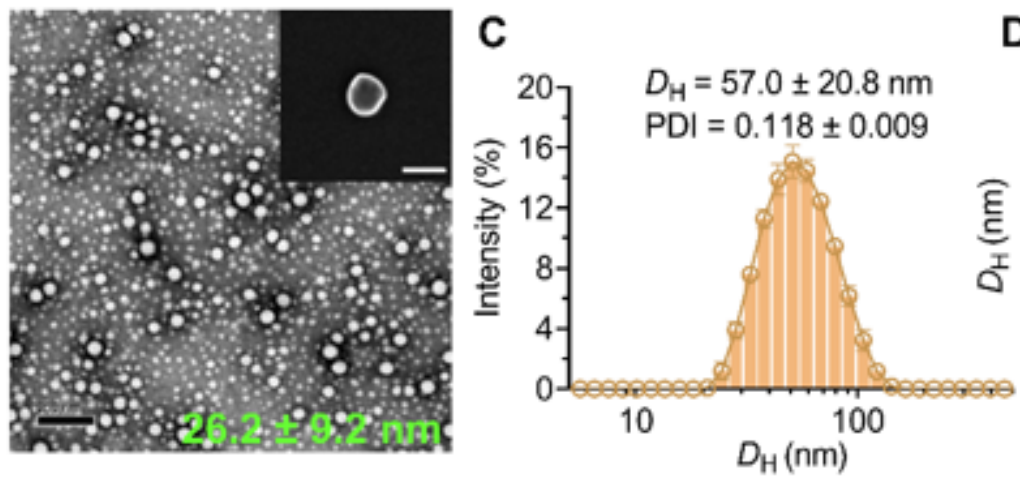

D

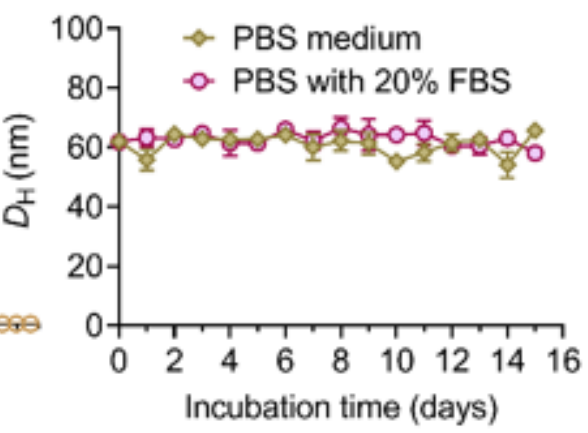

Figure 2

Preparation and characterization of OLA-CTX prodrug-loaded nanoparticles (NPs) using PEG5K-b-PLA8K. a Schematic illustration of the protocol for NP preparation. b Transmission electron microscopy (TEM) image of NPs in aqueous solution. Inset: NP characterized by scanning electron microscopy (SEM). c Size distribution of NPs measured by dynamic light scattering (DLS). d Variation of DH of NPs in PBS (pH $=7.4$ ) and in PBS containing 20\% FBS for 15 days. 
A

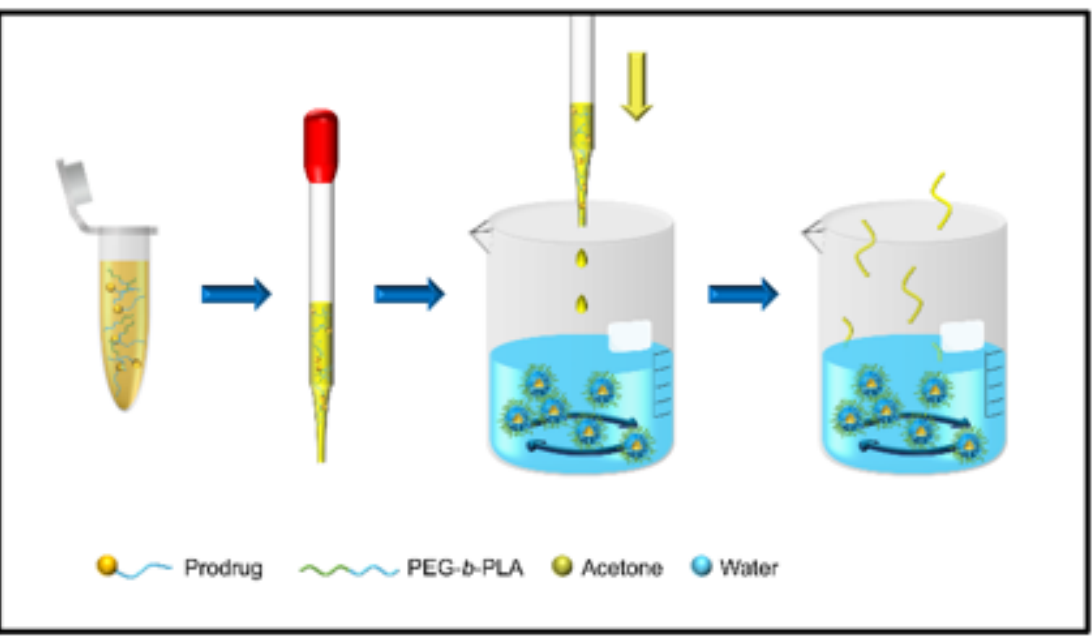

B
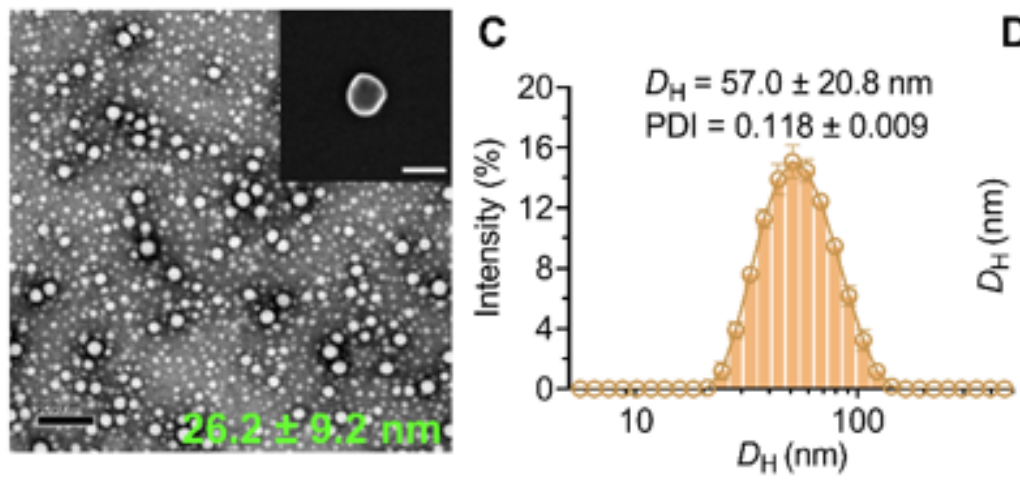

D

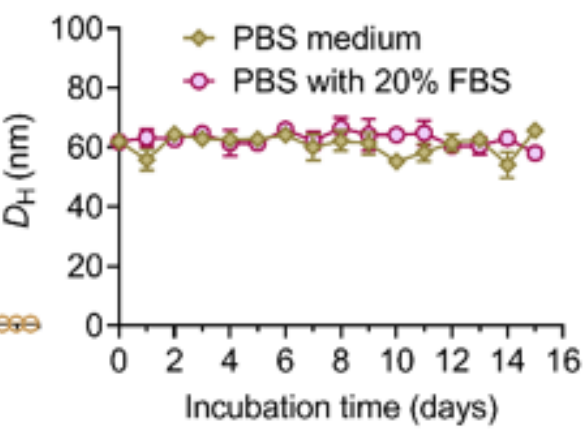

Figure 2

Preparation and characterization of OLA-CTX prodrug-loaded nanoparticles (NPs) using PEG5K-b-PLA8K. a Schematic illustration of the protocol for NP preparation. b Transmission electron microscopy (TEM) image of NPs in aqueous solution. Inset: NP characterized by scanning electron microscopy (SEM). c Size distribution of NPs measured by dynamic light scattering (DLS). d Variation of DH of NPs in PBS (pH $=7.4$ ) and in PBS containing 20\% FBS for 15 days. 
A

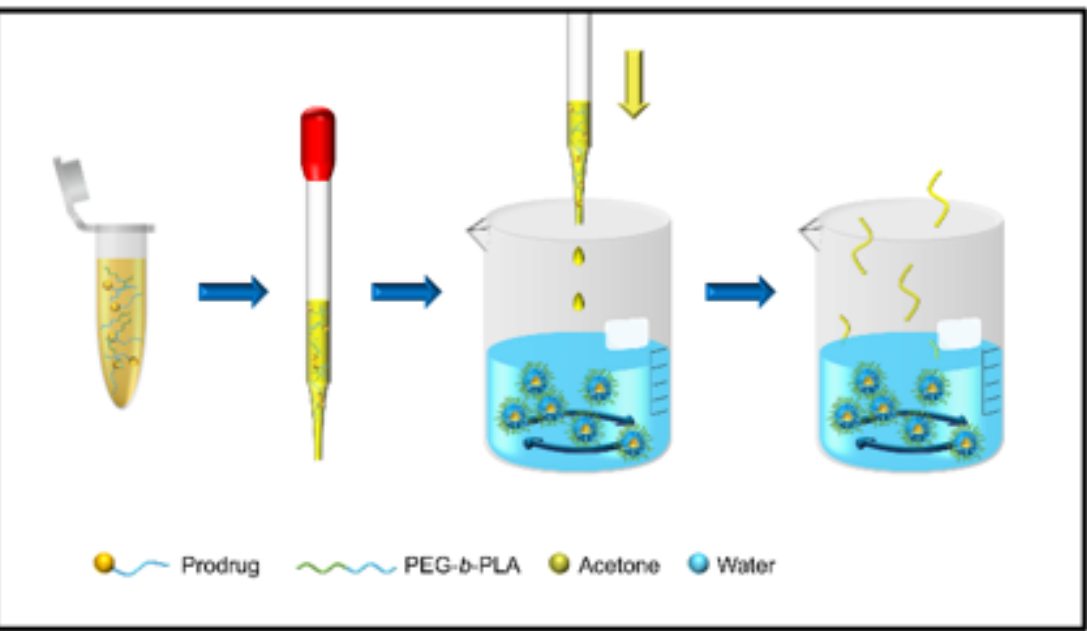

B
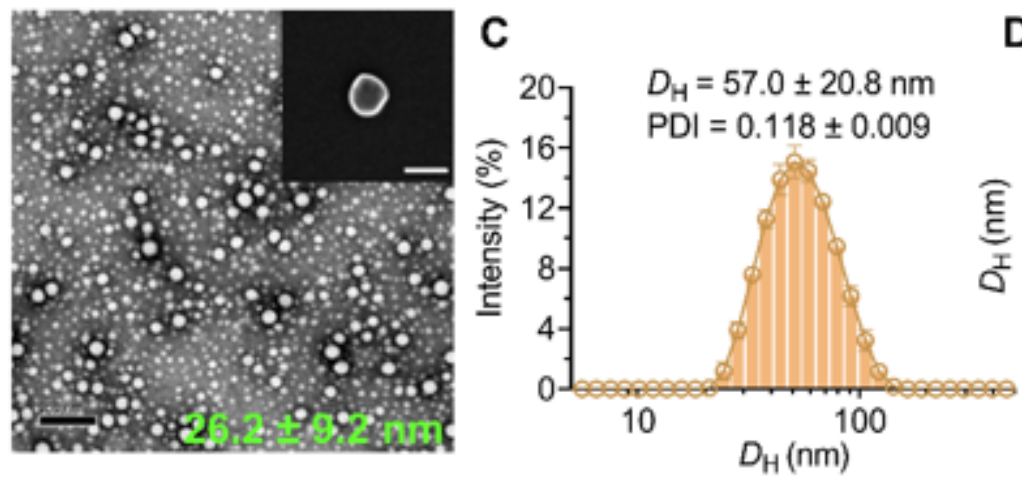

D

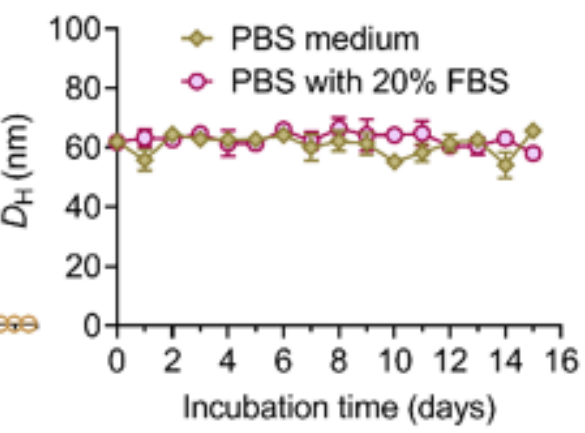

Figure 2

Preparation and characterization of OLA-CTX prodrug-loaded nanoparticles (NPs) using PEG5K-b-PLA8K. a Schematic illustration of the protocol for NP preparation. b Transmission electron microscopy (TEM) image of NPs in aqueous solution. Inset: NP characterized by scanning electron microscopy (SEM). C Size distribution of NPs measured by dynamic light scattering (DLS). d Variation of DH of NPs in PBS (pH $=7.4$ ) and in PBS containing 20\% FBS for 15 days. 

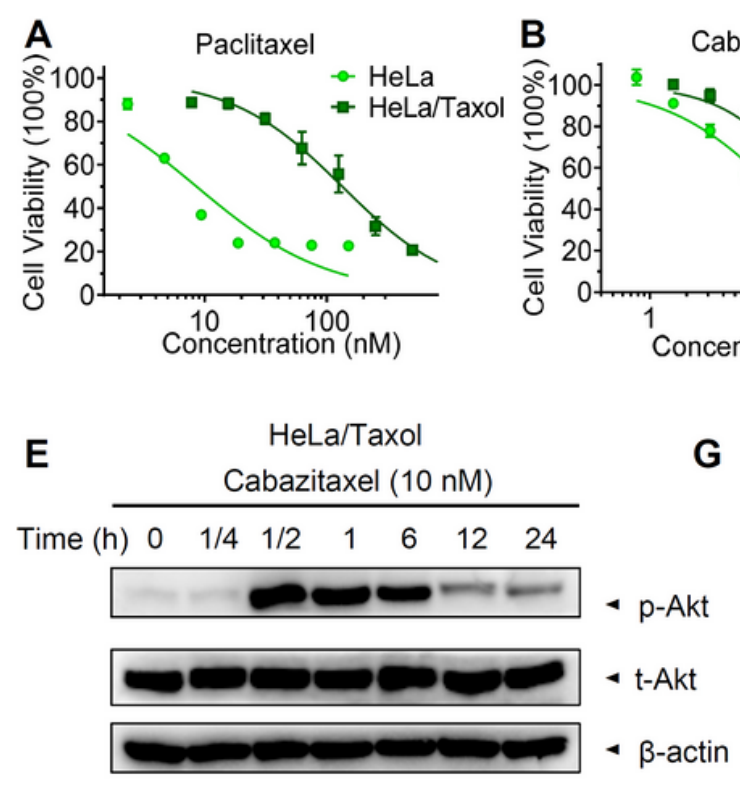

$\mathbf{F}$

A549/Taxol Cabazitaxel (10 nM)

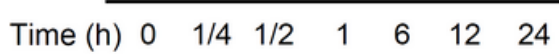

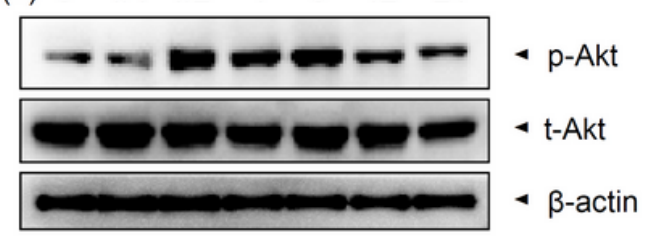

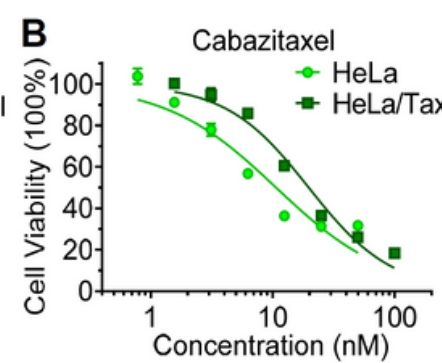

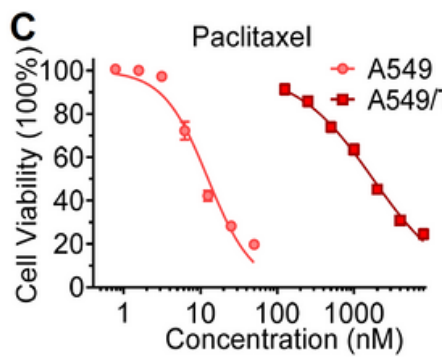

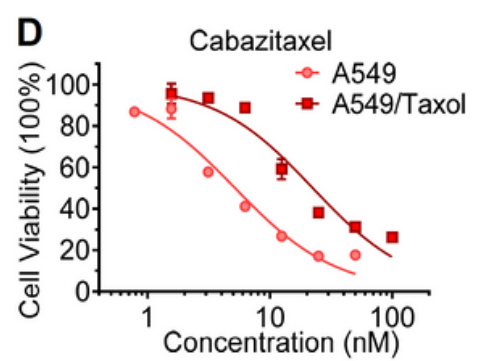

HeLa/Taxol

Cabazitaxel (10 nM)

MK-2206 $3 \mu \mathrm{M}$

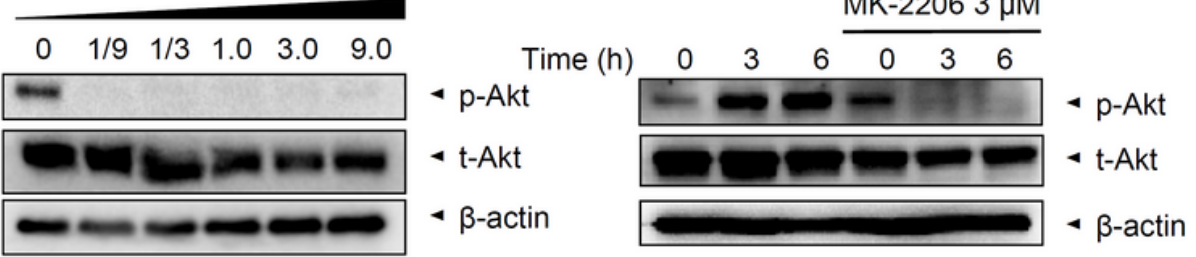

$\mathbf{J}$

A549/Taxol

A549/Taxol

Cabazitaxel (10 nM)

MK-2206 $(\mu \mathrm{M})$

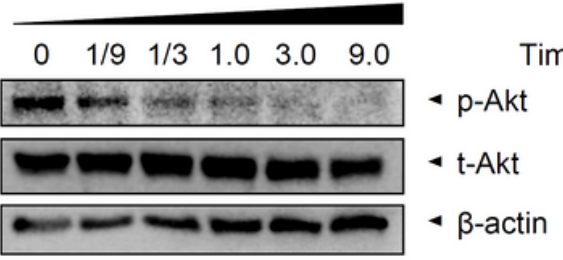

MK-2206 $3 \mu \mathrm{M}$

Time (h) $\begin{array}{llllll}0 & 3 & 6 & 0 & 3 & 6\end{array}$

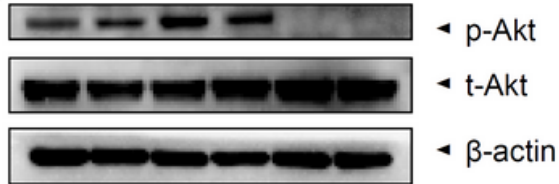

Figure 3

Cabazitaxel treatment triggers Akt activation in paclitaxel-resistant cells, which is reversed by the addition of MK-2206. a-d Cytotoxicity of paclitaxel $(a, c)$ and cabazitaxel $(b, d)$ against paclitaxel-sensitive and paclitaxel-resistant cells. e, $f$ Cabazitaxel treatment increases phosphorylated Akt (p-Akt) levels in paclitaxel-resistant HeLa/Taxol cells (e) and A549/Taxol cells (f). Cells were treated with cabazitaxel at $10 \mathrm{nmol} / \mathrm{L}$ for the indicated times. $\mathrm{g}$, h MK-2206 inhibits Akt signaling in HeLa/Taxol cells (g) and A549/Taxol cells (h). i, j MK-2206 significantly reversed cabazitaxel-induced phosphorylation of Akt in both cell lines. The results were obtained in three independent experiments. 

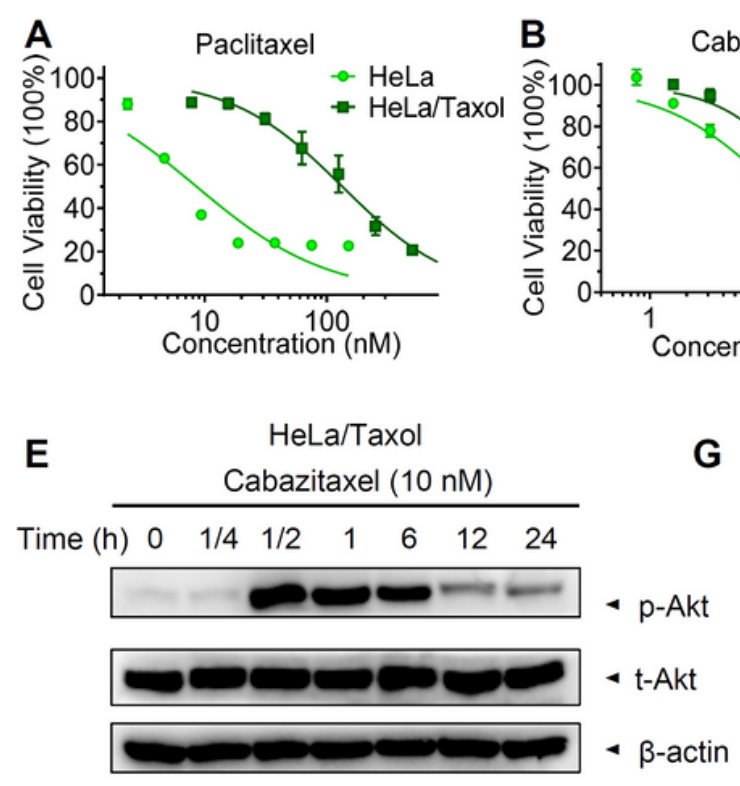

$\mathbf{F}$

A549/Taxol Cabazitaxel (10 nM)

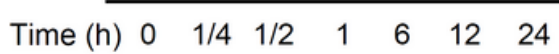

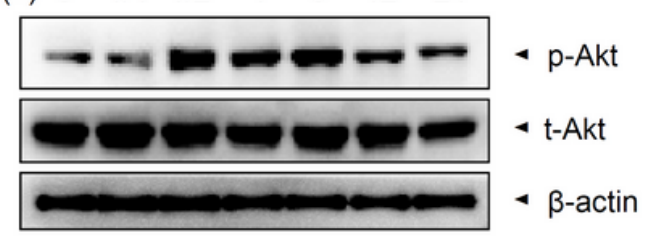

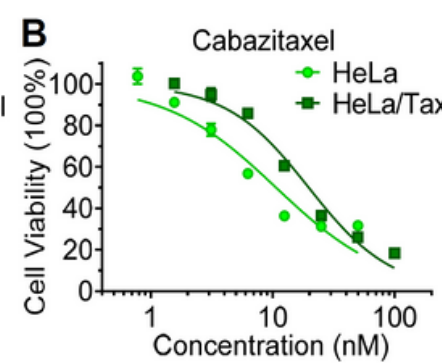

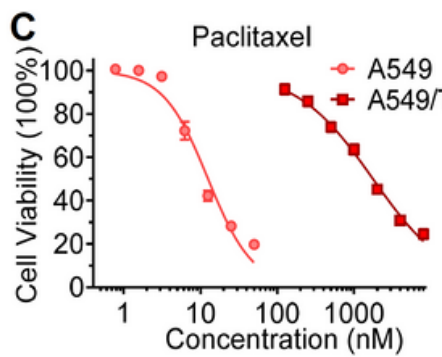

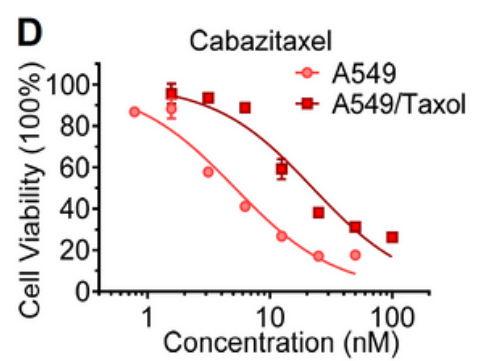

HeLa/Taxol

Cabazitaxel (10 nM)

MK-2206 $3 \mu \mathrm{M}$

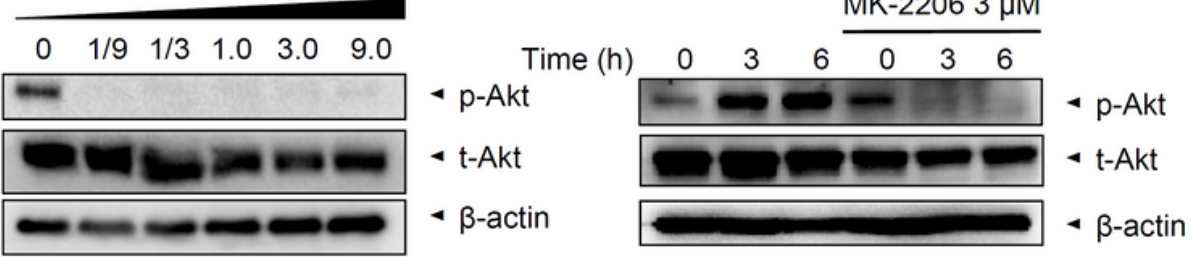

$\mathbf{J}$

A549/Taxol

A549/Taxol

Cabazitaxel (10 nM)

MK-2206 $(\mu \mathrm{M})$

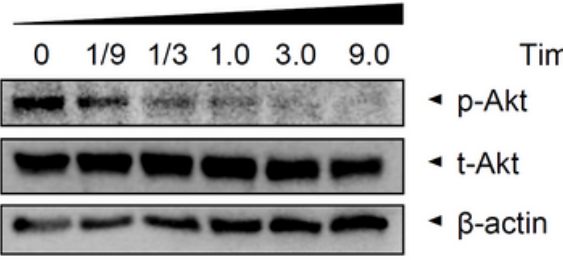

MK-2206 $3 \mu \mathrm{M}$

Time (h) $\begin{array}{llllll}0 & 3 & 6 & 0 & 3 & 6\end{array}$

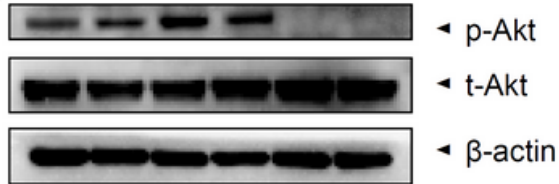

Figure 3

Cabazitaxel treatment triggers Akt activation in paclitaxel-resistant cells, which is reversed by the addition of MK-2206. a-d Cytotoxicity of paclitaxel $(a, c)$ and cabazitaxel $(b, d)$ against paclitaxel-sensitive and paclitaxel-resistant cells. e, $f$ Cabazitaxel treatment increases phosphorylated Akt (p-Akt) levels in paclitaxel-resistant HeLa/Taxol cells (e) and A549/Taxol cells (f). Cells were treated with cabazitaxel at $10 \mathrm{nmol} / \mathrm{L}$ for the indicated times. $\mathrm{g}$, h MK-2206 inhibits Akt signaling in HeLa/Taxol cells (g) and A549/Taxol cells (h). i, j MK-2206 significantly reversed cabazitaxel-induced phosphorylation of Akt in both cell lines. The results were obtained in three independent experiments. 

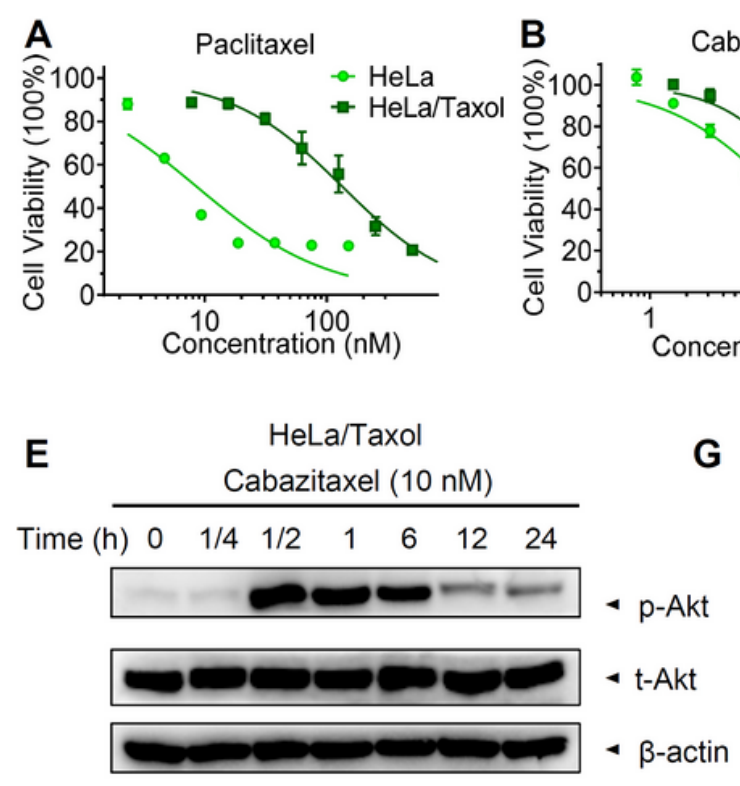

$\mathbf{F}$

A549/Taxol Cabazitaxel (10 nM)

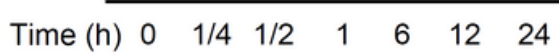

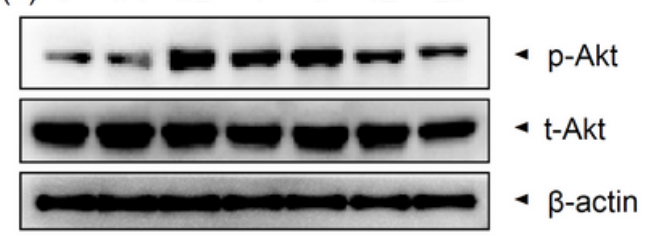

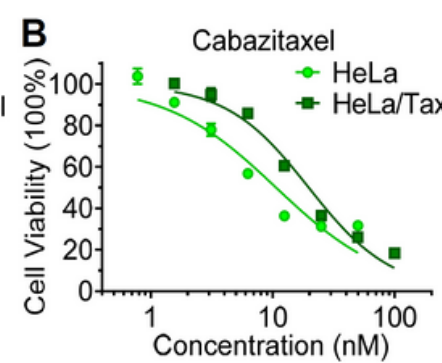

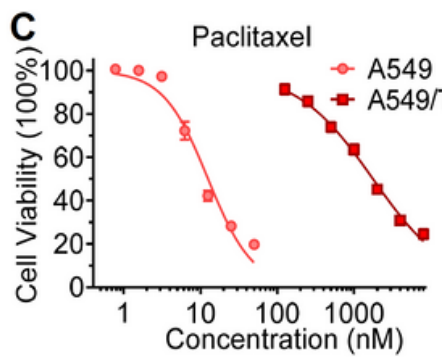

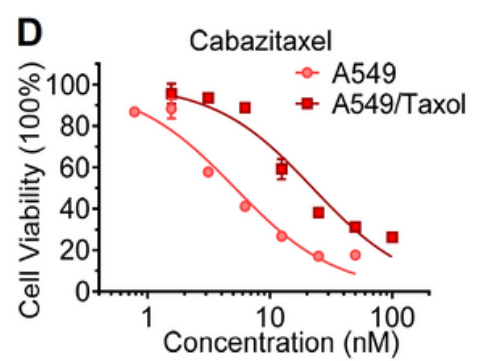

HeLa/Taxol

Cabazitaxel (10 nM)

MK-2206 $3 \mu \mathrm{M}$

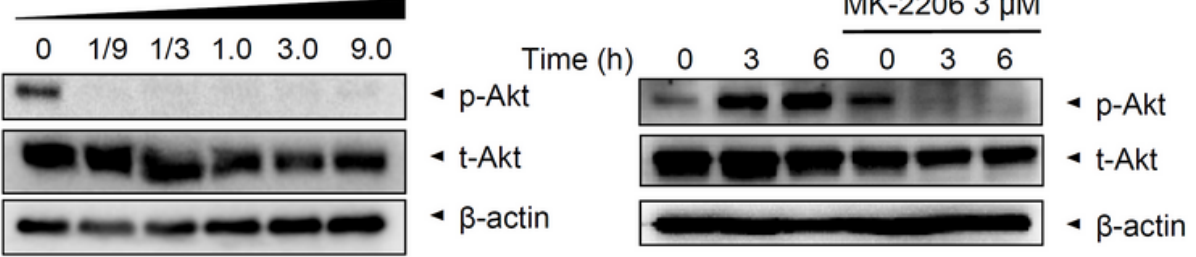

$\mathbf{J}$

A549/Taxol

A549/Taxol

Cabazitaxel (10 nM)

MK-2206 $(\mu \mathrm{M})$

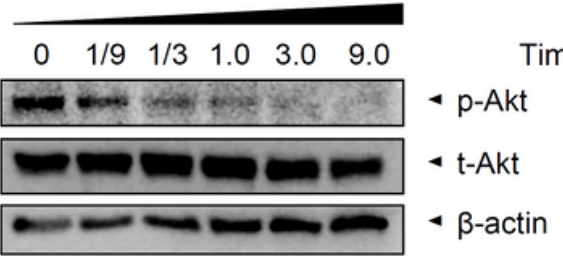

MK-2206 $3 \mu \mathrm{M}$

Time (h) $\begin{array}{llllll}0 & 3 & 6 & 0 & 3 & 6\end{array}$

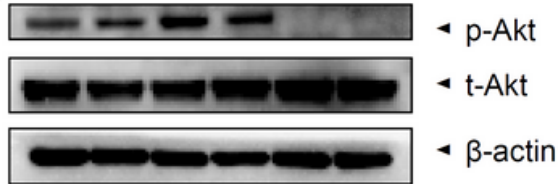

Figure 3

Cabazitaxel treatment triggers Akt activation in paclitaxel-resistant cells, which is reversed by the addition of MK-2206. a-d Cytotoxicity of paclitaxel $(a, c)$ and cabazitaxel $(b, d)$ against paclitaxel-sensitive and paclitaxel-resistant cells. e, $f$ Cabazitaxel treatment increases phosphorylated Akt (p-Akt) levels in paclitaxel-resistant HeLa/Taxol cells (e) and A549/Taxol cells (f). Cells were treated with cabazitaxel at $10 \mathrm{nmol} / \mathrm{L}$ for the indicated times. $\mathrm{g}$, h MK-2206 inhibits Akt signaling in HeLa/Taxol cells (g) and A549/Taxol cells (h). i, j MK-2206 significantly reversed cabazitaxel-induced phosphorylation of Akt in both cell lines. The results were obtained in three independent experiments. 

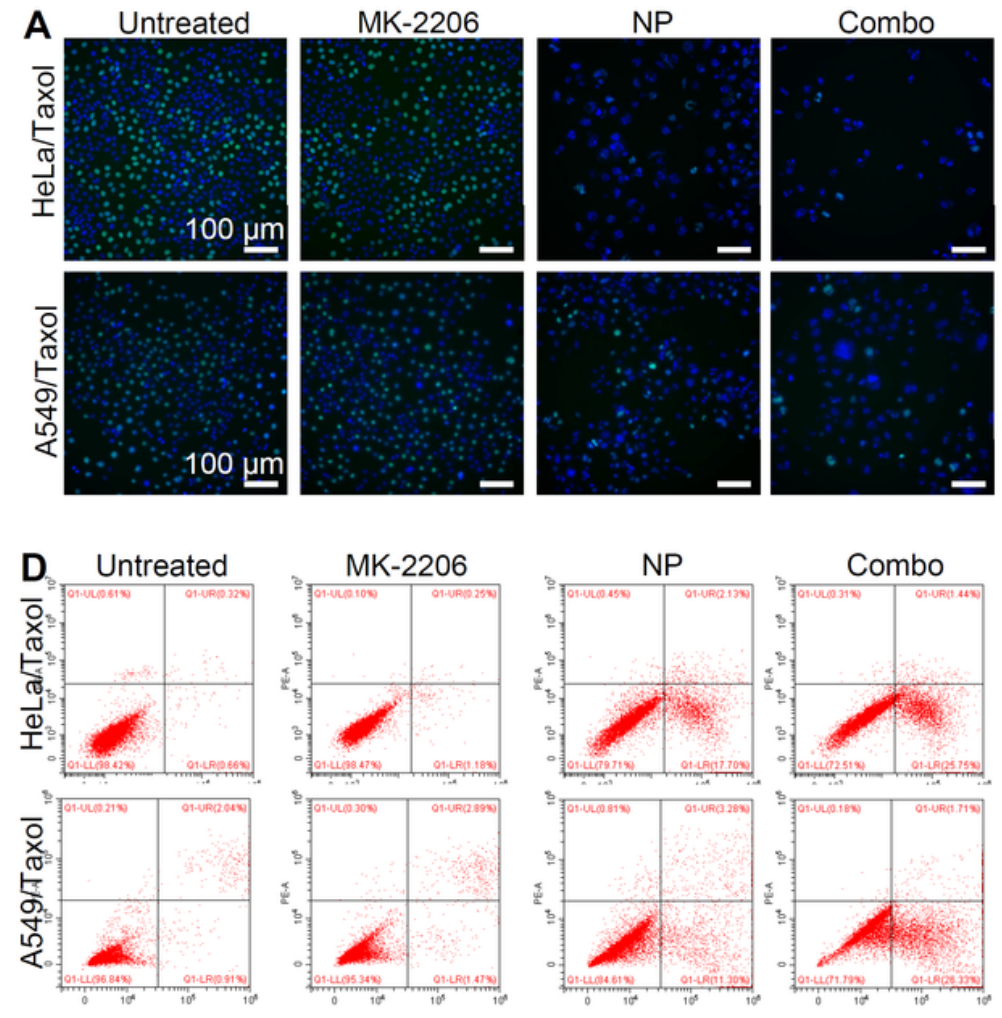

$\mathrm{HeLa} / \mathrm{Taxol}$

F

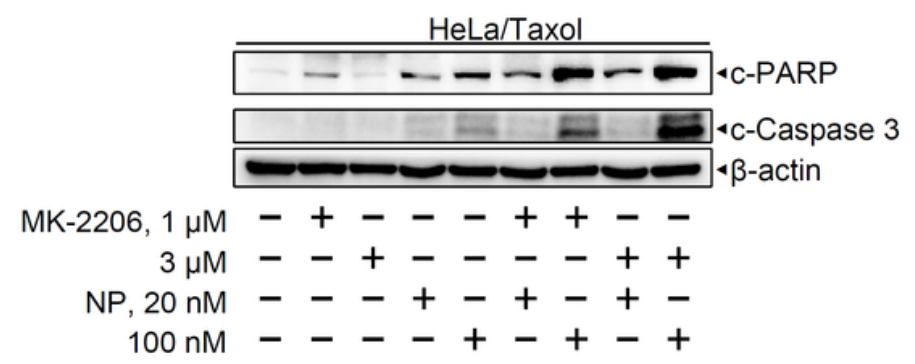

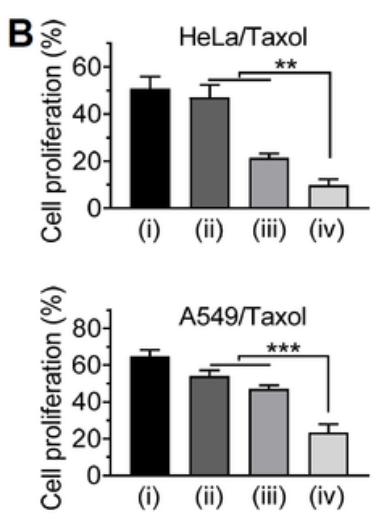
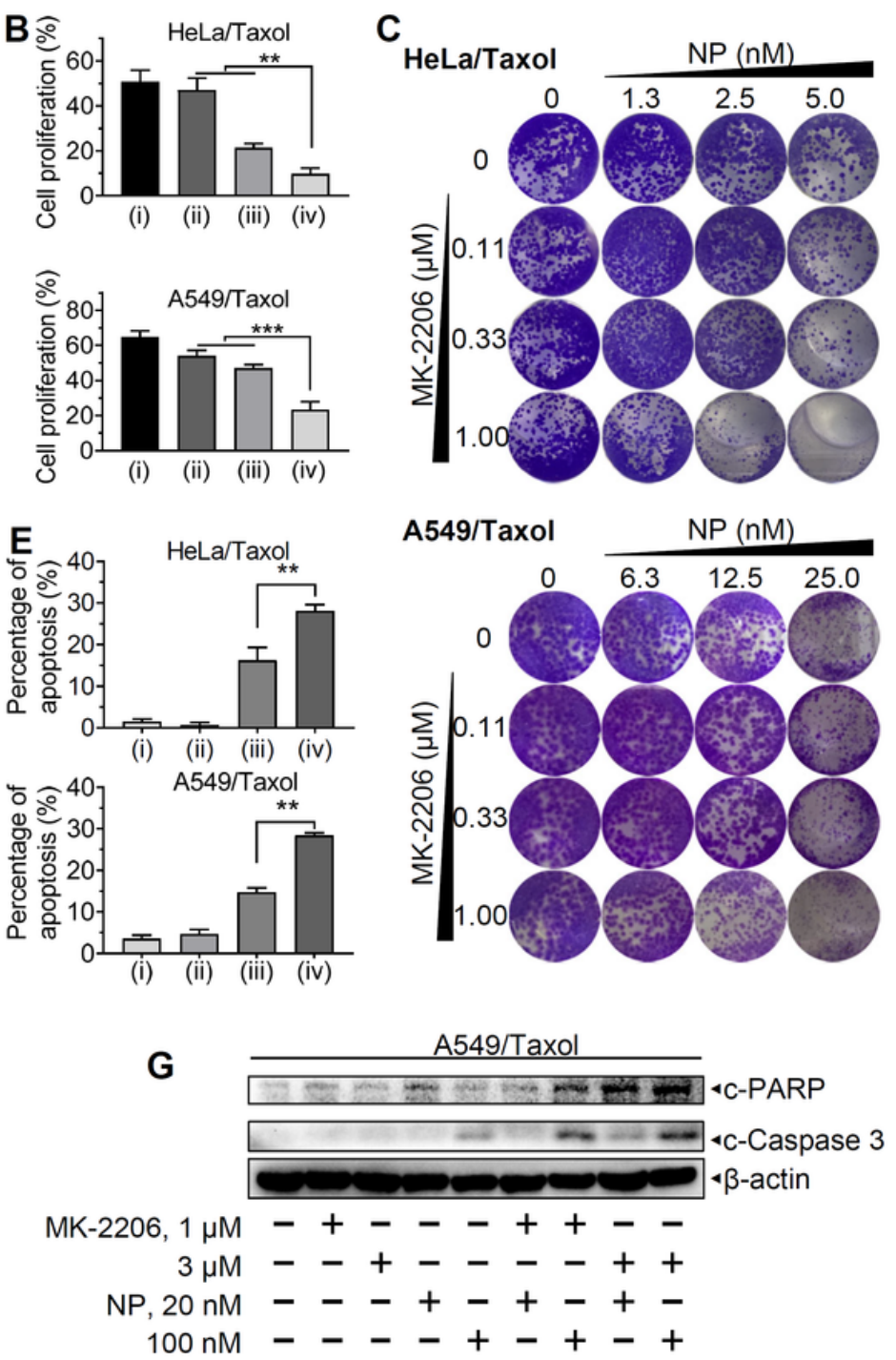

\section{Figure 4}

The combination of MK-2206 and cabazitaxel-NPs exhibits enhanced anticancer effects in taxaneresistant cells. a Representative images show the proliferation profiles of resistant cells, as measured by a Click-iT EdU assay. Cells were treated with DMSO, MK-2206, cabazitaxel-NPs, or the combination regimen for 48 h. b Quantification of the EdU-positive cells indicated that cotreatment with MK-2206 and cabazitaxel-NPs markedly decreased cell proliferation in the observed cells. c The effect of various concentrations of cabazitaxel-NPs and/or MK-2206 on colony formation in HeLa/Taxol (upper) and A549/Taxol (lower) cells. d Representative apoptotic profiles of HeLa/Taxol (upper) and A549/Taxol (lower) cells treated with DMSO, MK-2206 $(3 \mu \mathrm{M})$, cabazitaxel-NPs $(100 \mathrm{nM})$, or the combination for $48 \mathrm{~h}$ as assessed by Annexin V-FITC-PI staining and flow cytometry. e Quantification of apoptotic cell ratios from three separate experiments. The data are presented as the means $\pm S D ; * \star p<0.01$, $* \star * p<0.001$. f, $g$ Western blot analysis of cleaved PARP (c-PARP) and cleaved caspase-3 (c-caspase-3) in HeLa/Taxol (f) and A549/Taxol (g) cells after different treatments for $48 \mathrm{~h}$. 

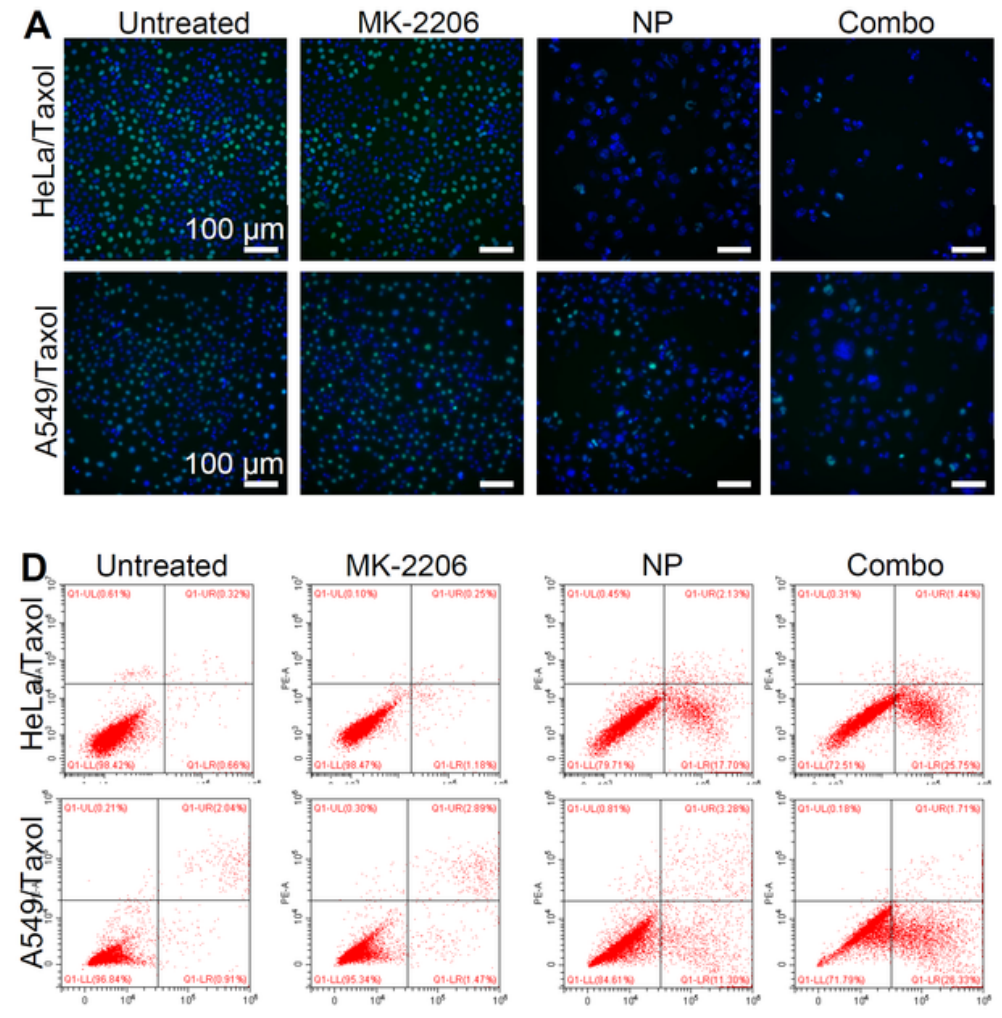

$\mathrm{HeLa} / \mathrm{Taxol}$

F

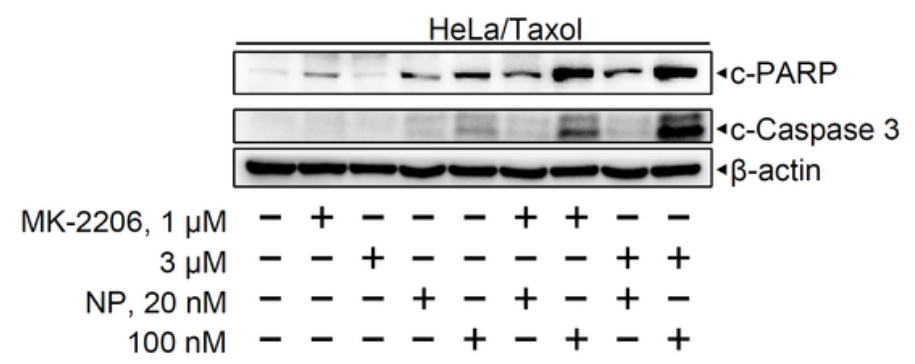

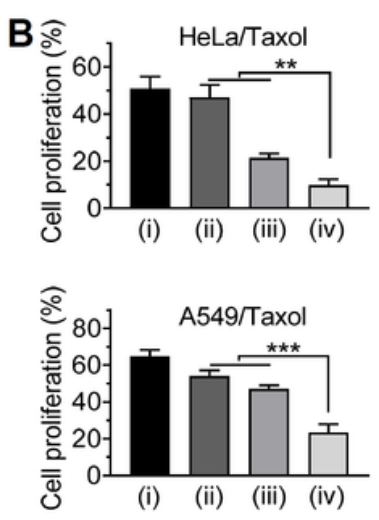
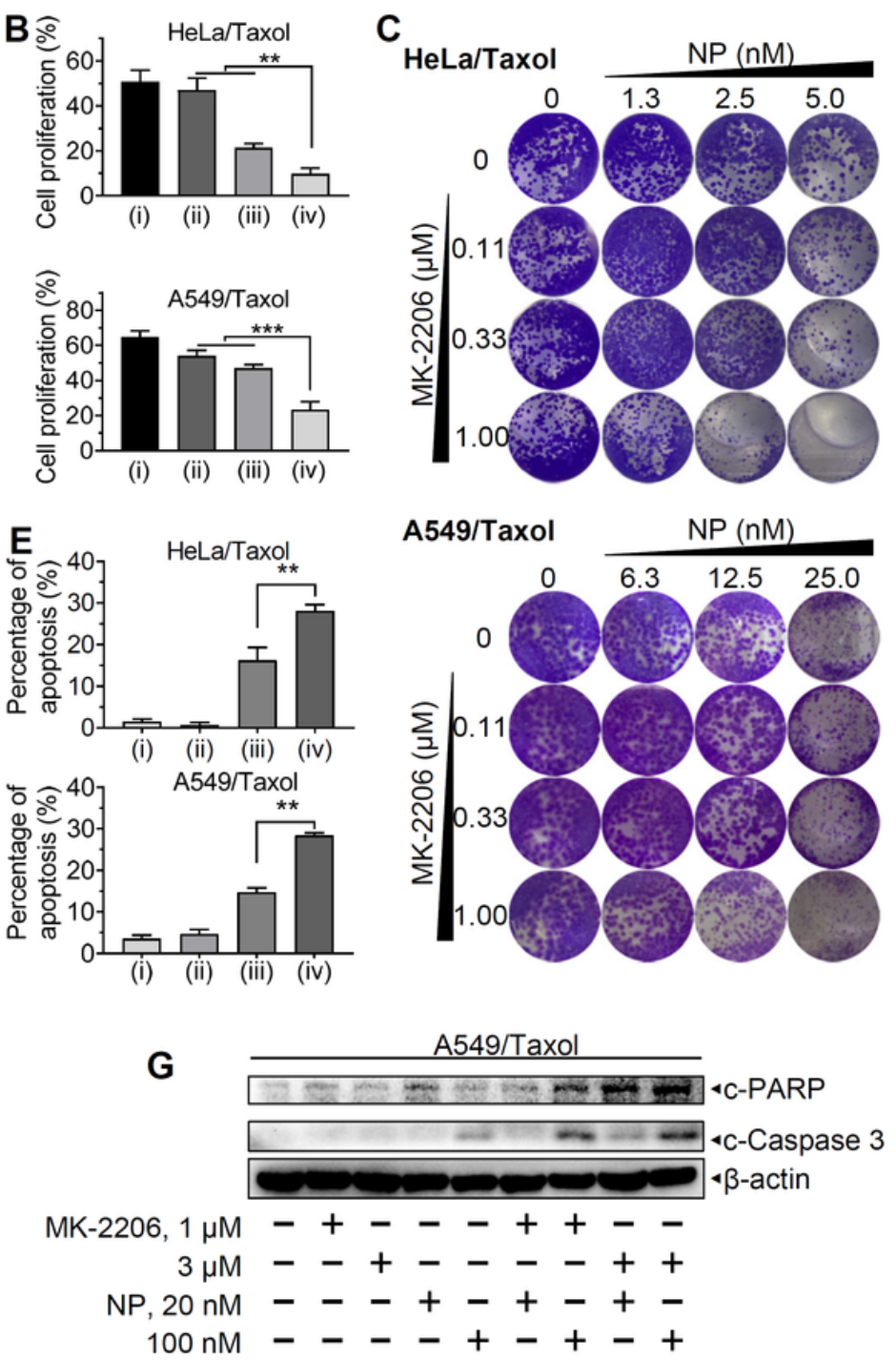

\section{Figure 4}

The combination of MK-2206 and cabazitaxel-NPs exhibits enhanced anticancer effects in taxaneresistant cells. a Representative images show the proliferation profiles of resistant cells, as measured by a Click-iT EdU assay. Cells were treated with DMSO, MK-2206, cabazitaxel-NPs, or the combination regimen for 48 h. b Quantification of the EdU-positive cells indicated that cotreatment with MK-2206 and cabazitaxel-NPs markedly decreased cell proliferation in the observed cells. c The effect of various concentrations of cabazitaxel-NPs and/or MK-2206 on colony formation in HeLa/Taxol (upper) and A549/Taxol (lower) cells. d Representative apoptotic profiles of HeLa/Taxol (upper) and A549/Taxol (lower) cells treated with DMSO, MK-2206 $(3 \mu \mathrm{M})$, cabazitaxel-NPs $(100 \mathrm{nM})$, or the combination for $48 \mathrm{~h}$ as assessed by Annexin V-FITC-PI staining and flow cytometry. e Quantification of apoptotic cell ratios from three separate experiments. The data are presented as the means $\pm S D ; * \star p<0.01$, $* \star * p<0.001$. f, $g$ Western blot analysis of cleaved PARP (c-PARP) and cleaved caspase-3 (c-caspase-3) in HeLa/Taxol (f) and A549/Taxol (g) cells after different treatments for $48 \mathrm{~h}$. 

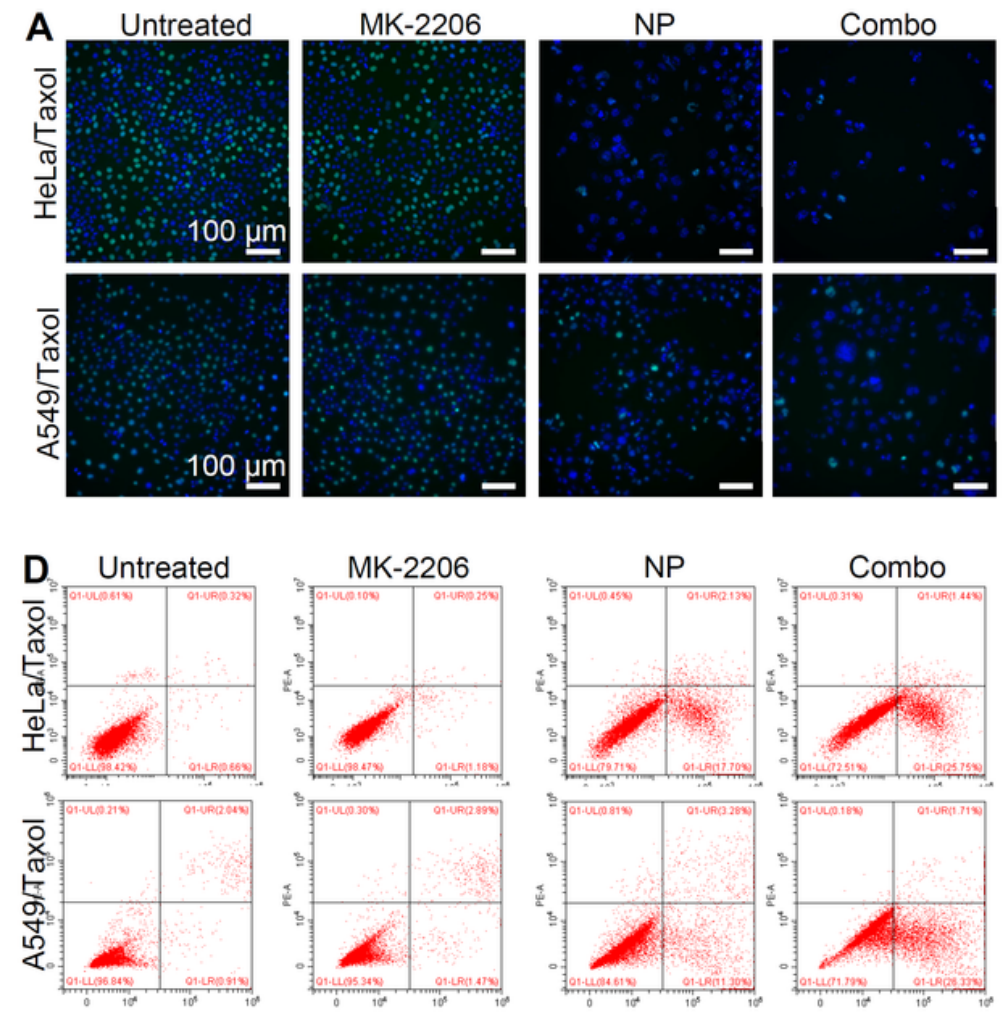

$\mathrm{HeLa} / \mathrm{Taxol}$

F

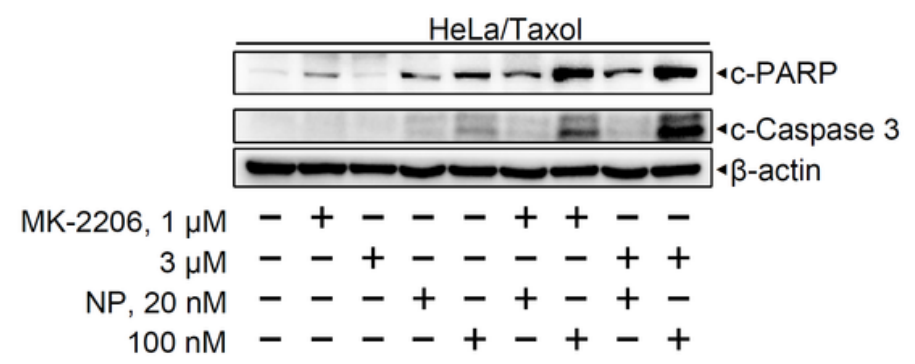

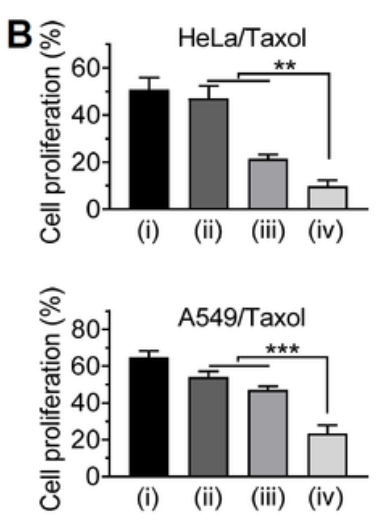
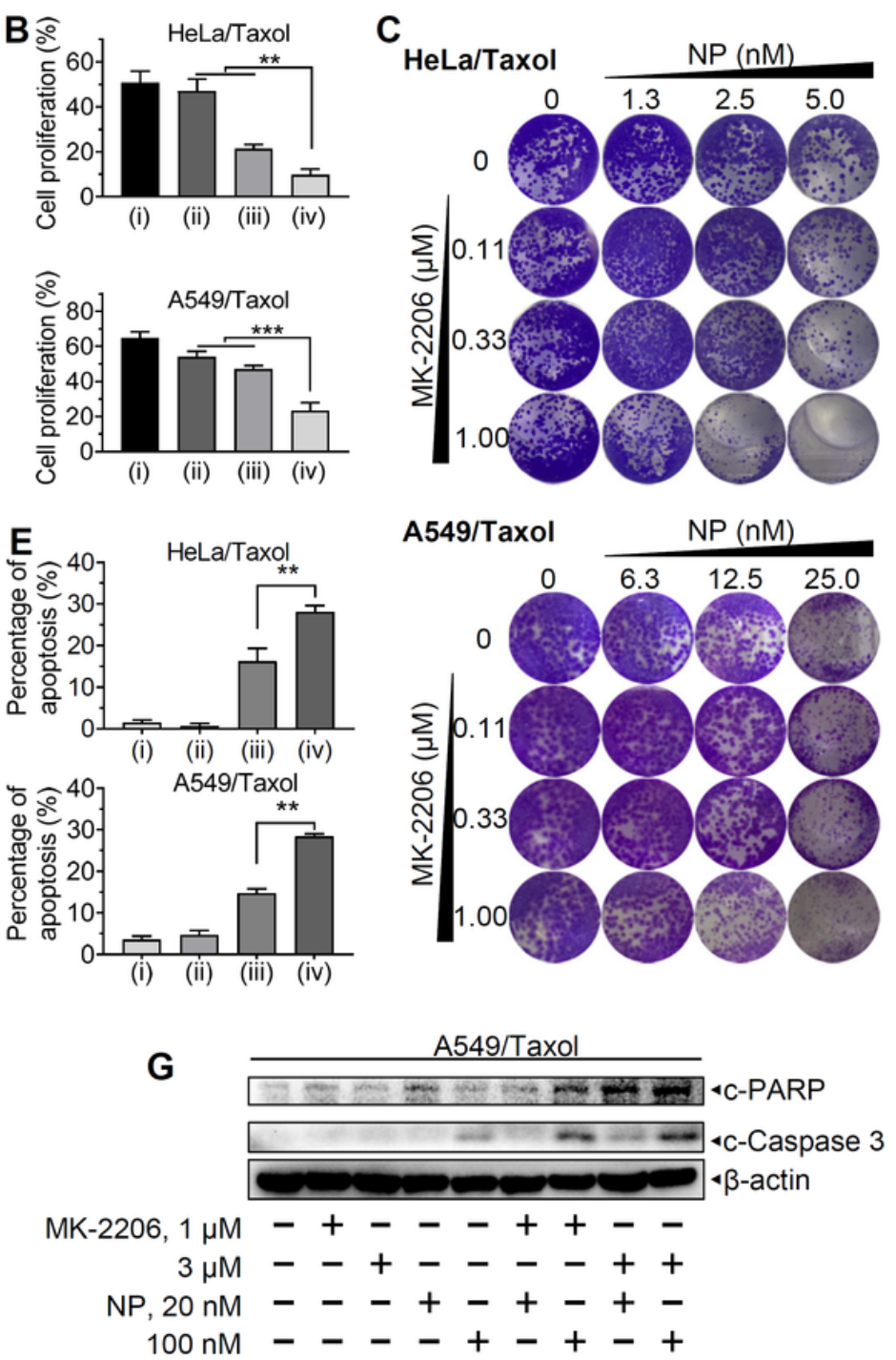

\section{Figure 4}

The combination of MK-2206 and cabazitaxel-NPs exhibits enhanced anticancer effects in taxaneresistant cells. a Representative images show the proliferation profiles of resistant cells, as measured by a Click-iT EdU assay. Cells were treated with DMSO, MK-2206, cabazitaxel-NPs, or the combination regimen for 48 h. b Quantification of the EdU-positive cells indicated that cotreatment with MK-2206 and cabazitaxel-NPs markedly decreased cell proliferation in the observed cells. c The effect of various concentrations of cabazitaxel-NPs and/or MK-2206 on colony formation in HeLa/Taxol (upper) and A549/Taxol (lower) cells. d Representative apoptotic profiles of HeLa/Taxol (upper) and A549/Taxol (lower) cells treated with DMSO, MK-2206 $(3 \mu \mathrm{M})$, cabazitaxel-NPs $(100 \mathrm{nM})$, or the combination for $48 \mathrm{~h}$ as assessed by Annexin V-FITC-PI staining and flow cytometry. e Quantification of apoptotic cell ratios from three separate experiments. The data are presented as the means $\pm S D ; * \star p<0.01$, $* \star * p<0.001$. f, $g$ Western blot analysis of cleaved PARP (c-PARP) and cleaved caspase-3 (c-caspase-3) in HeLa/Taxol (f) and A549/Taxol (g) cells after different treatments for $48 \mathrm{~h}$. 

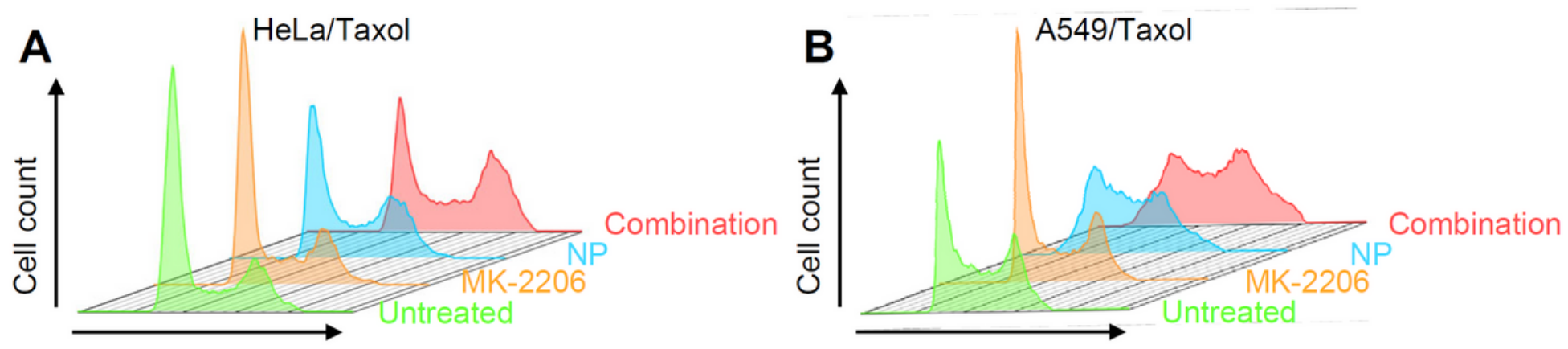

PI signal

HeLa/Taxol
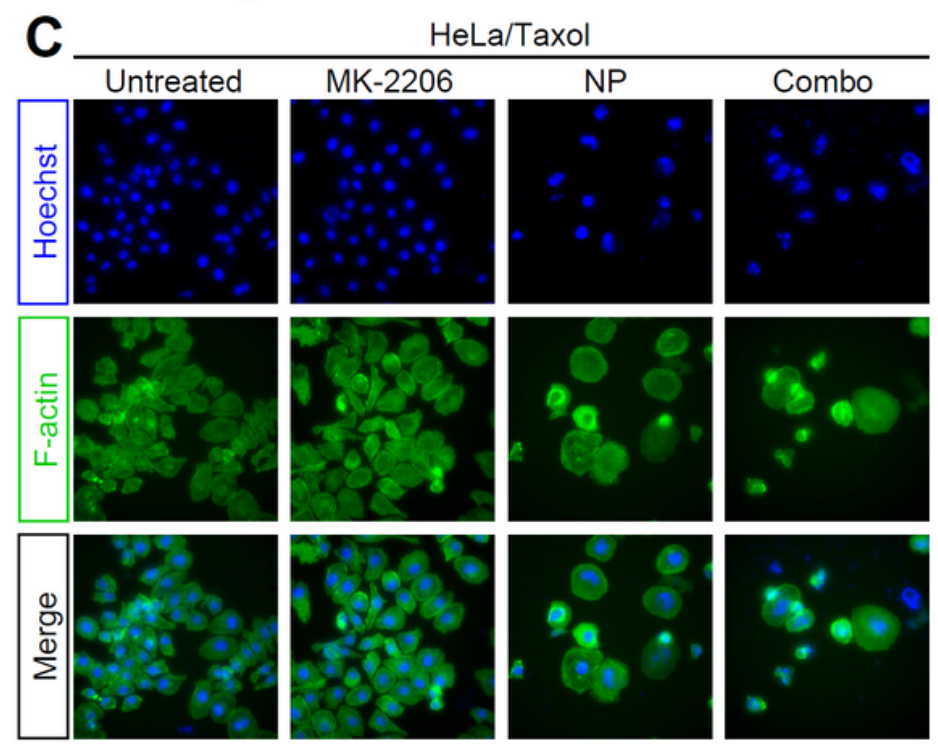

D

PI signal
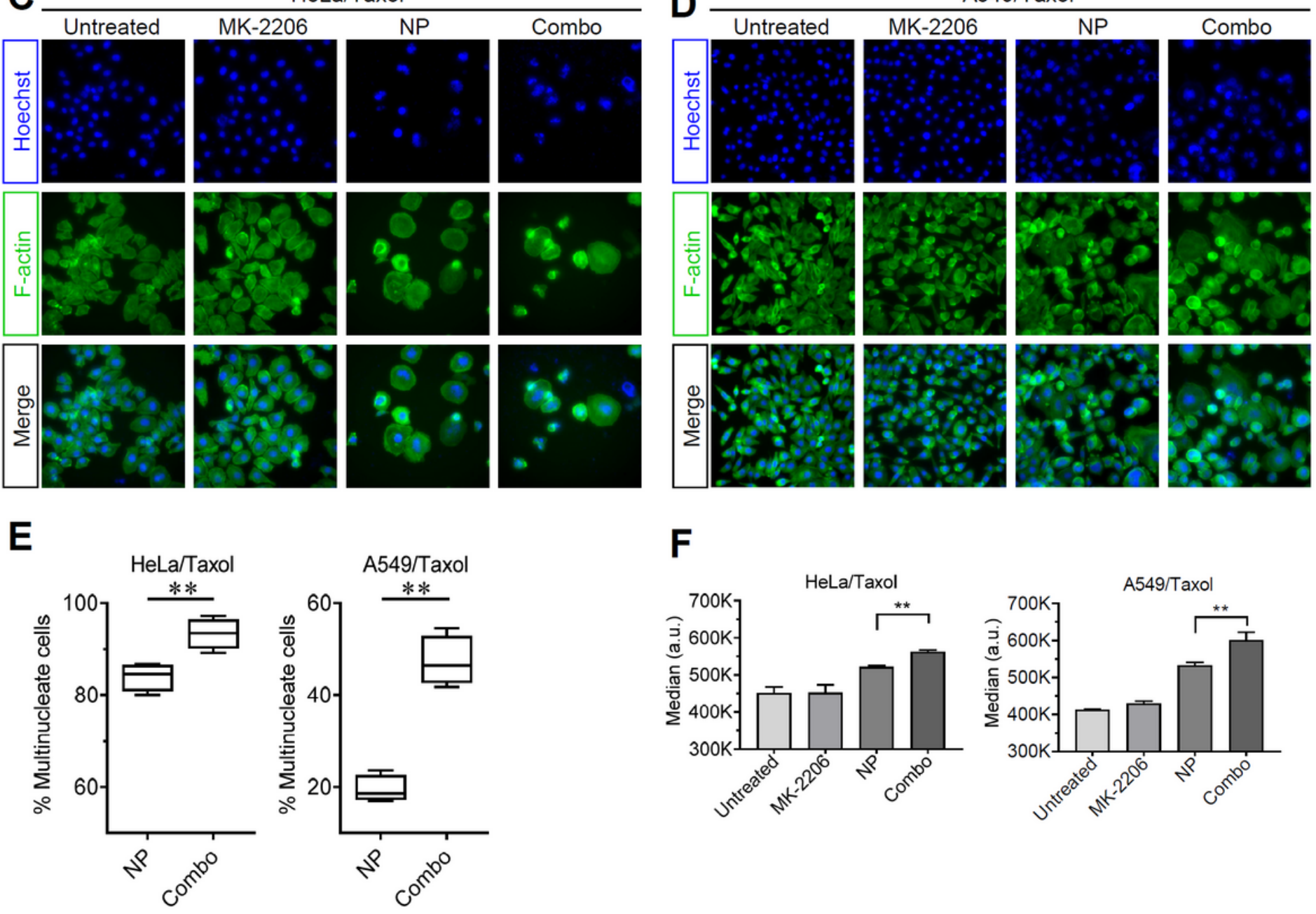

$\mathbf{F}$
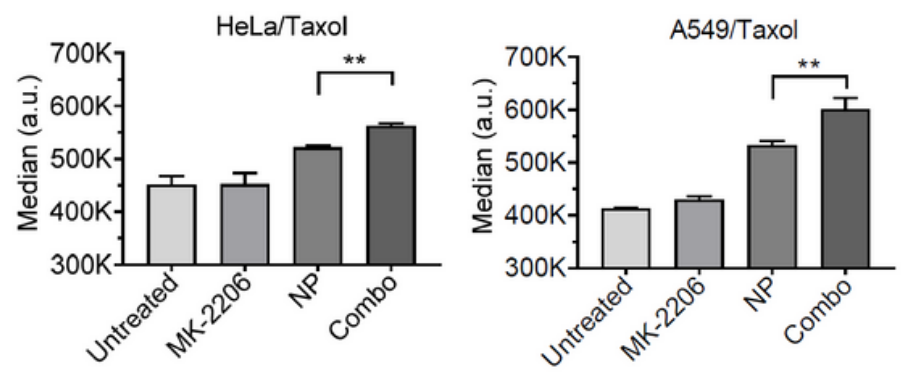

Figure 5

MK-2206 enhances the proportion of cell cycle arrest mediated by cabazitaxel-NPs. a, b Cell cycle profiles of HeLa/Taxol and A549/Taxol cells exposed to MK-2206 and/or cabazitaxel-NPs. c, d Staining with Hoechst and Alexa Fluor 488-labeled phalloidin reveals alternations in nuclear morphology and cytoplasmic volume in HeLa/Taxol cells (c) and A549/Taxol cells (d) after different treatments. e Box whisker plots indicate the fraction of the cells with abnormal mitoses (e.g., multinuclear defects) after exposure to cabazitaxel-NPs or the combination regimen. $f$ Forward scattering shows the heterogeneity in cell size after various treatments. The data are presented as the means \pm SD; $* \star p<0.01$. 

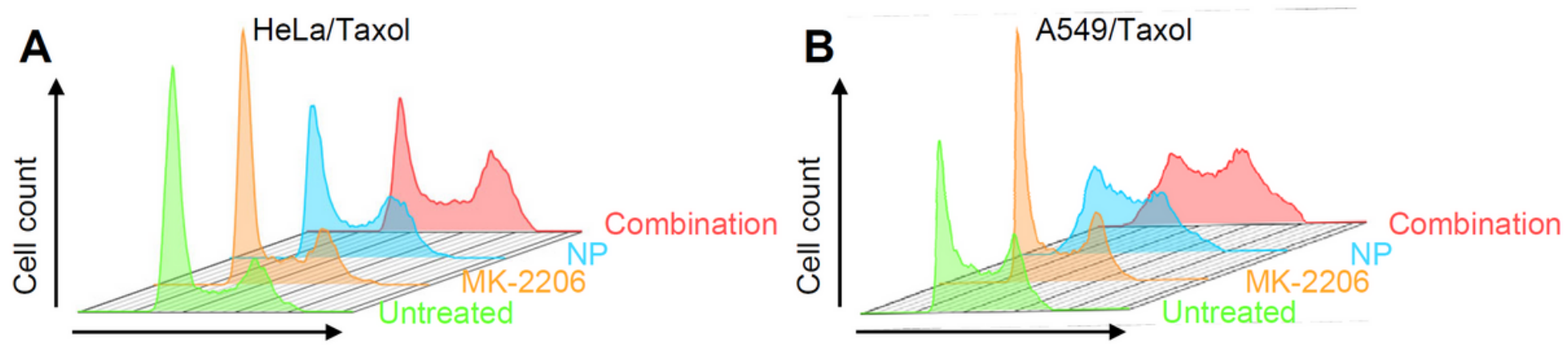

PI signal

HeLa/Taxol
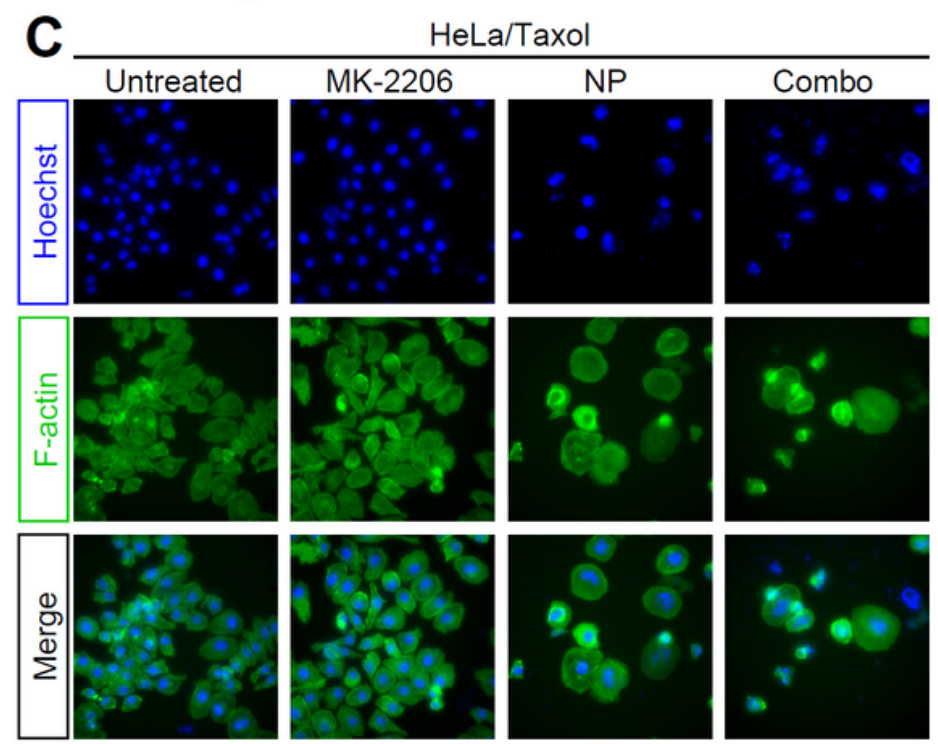

D

PI signal
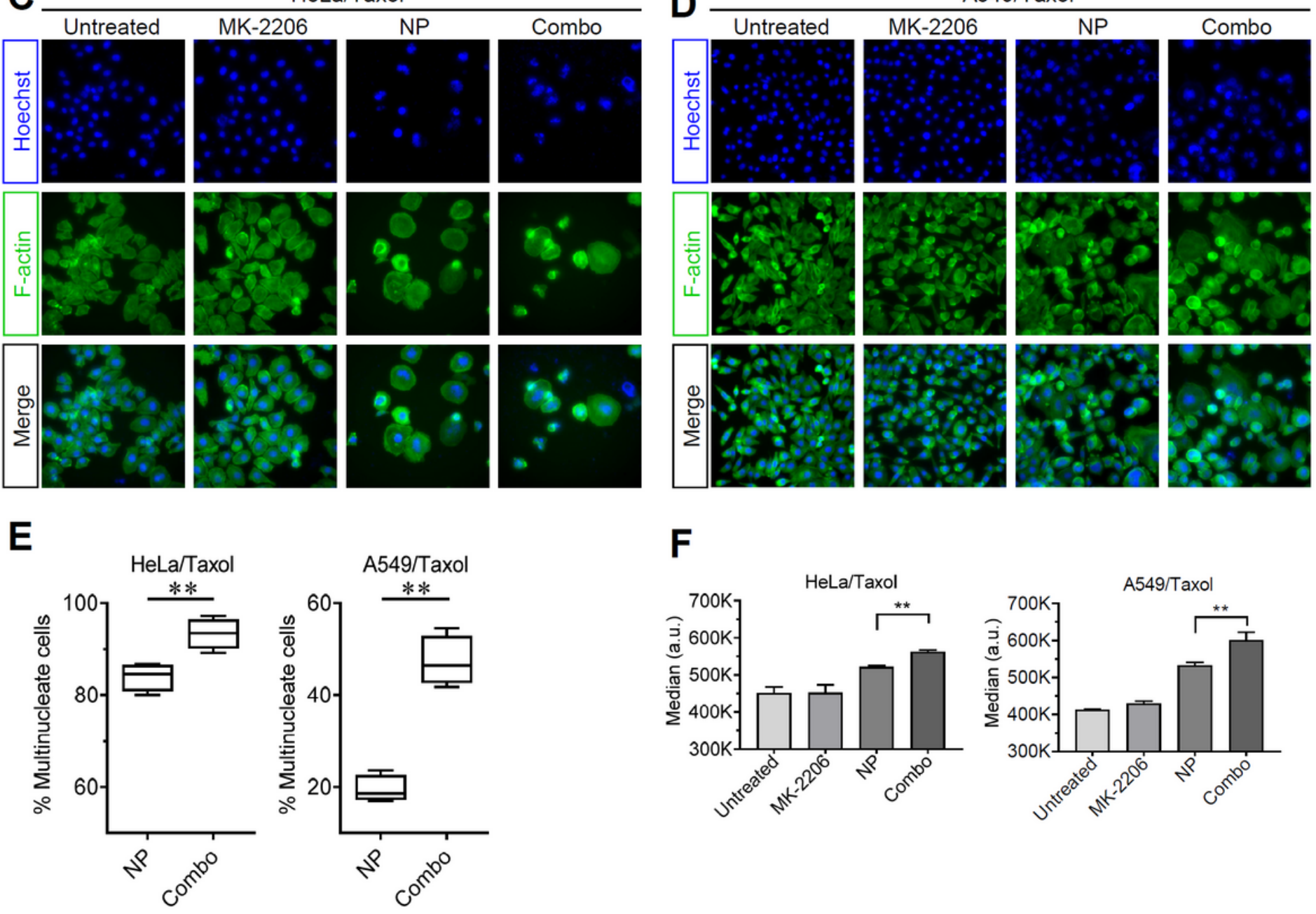

$\mathbf{F}$
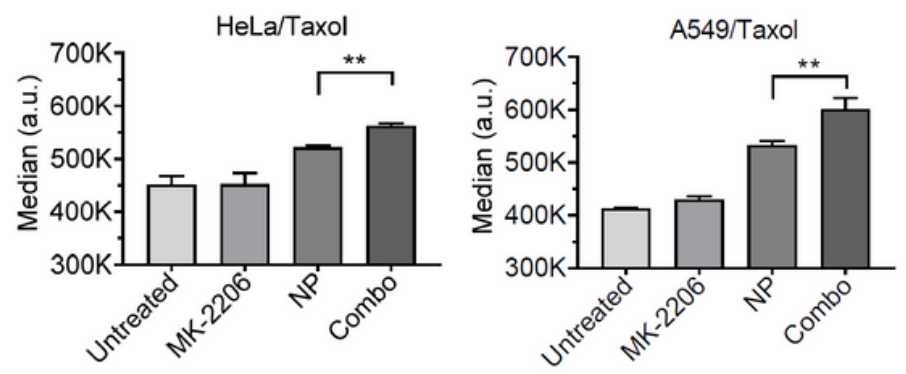

Figure 5

MK-2206 enhances the proportion of cell cycle arrest mediated by cabazitaxel-NPs. a, b Cell cycle profiles of HeLa/Taxol and A549/Taxol cells exposed to MK-2206 and/or cabazitaxel-NPs. c, d Staining with Hoechst and Alexa Fluor 488-labeled phalloidin reveals alternations in nuclear morphology and cytoplasmic volume in HeLa/Taxol cells (c) and A549/Taxol cells (d) after different treatments. e Box whisker plots indicate the fraction of the cells with abnormal mitoses (e.g., multinuclear defects) after exposure to cabazitaxel-NPs or the combination regimen. $f$ Forward scattering shows the heterogeneity in cell size after various treatments. The data are presented as the means \pm SD; $* \star p<0.01$. 

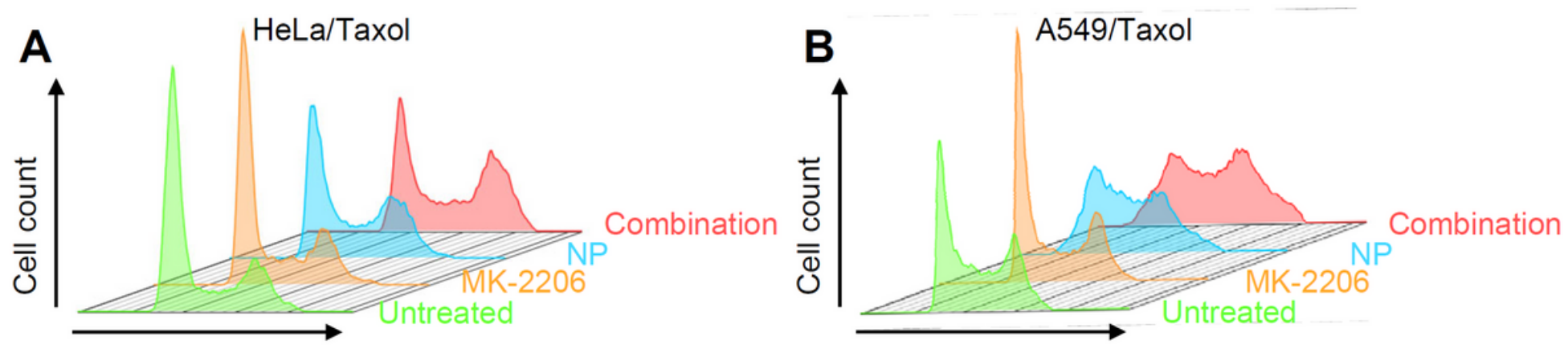

PI signal

HeLa/Taxol
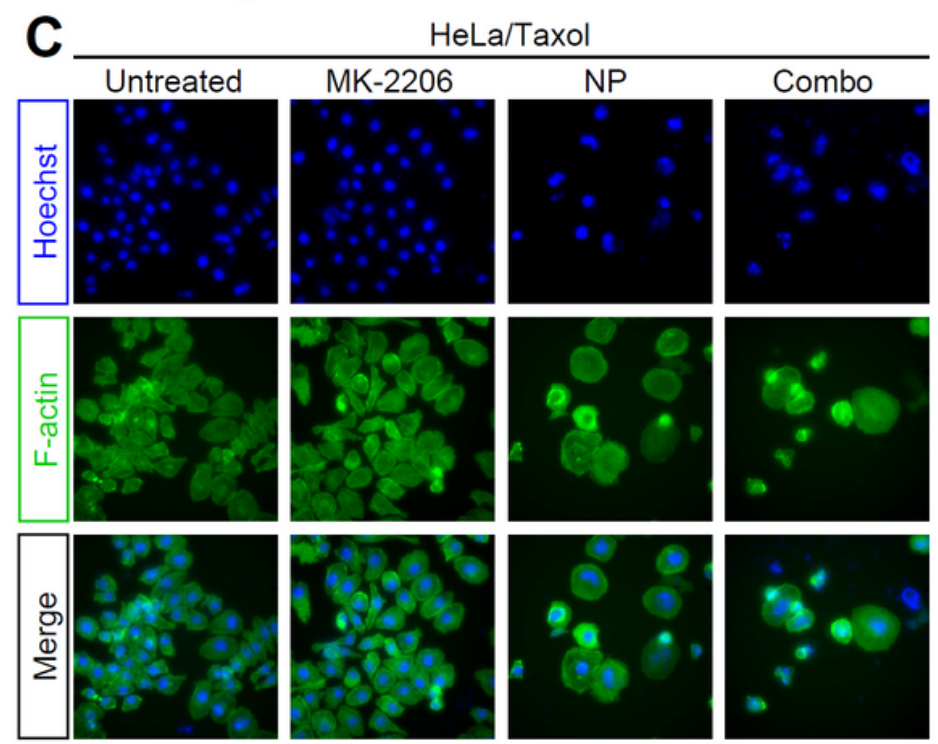

D

PI signal
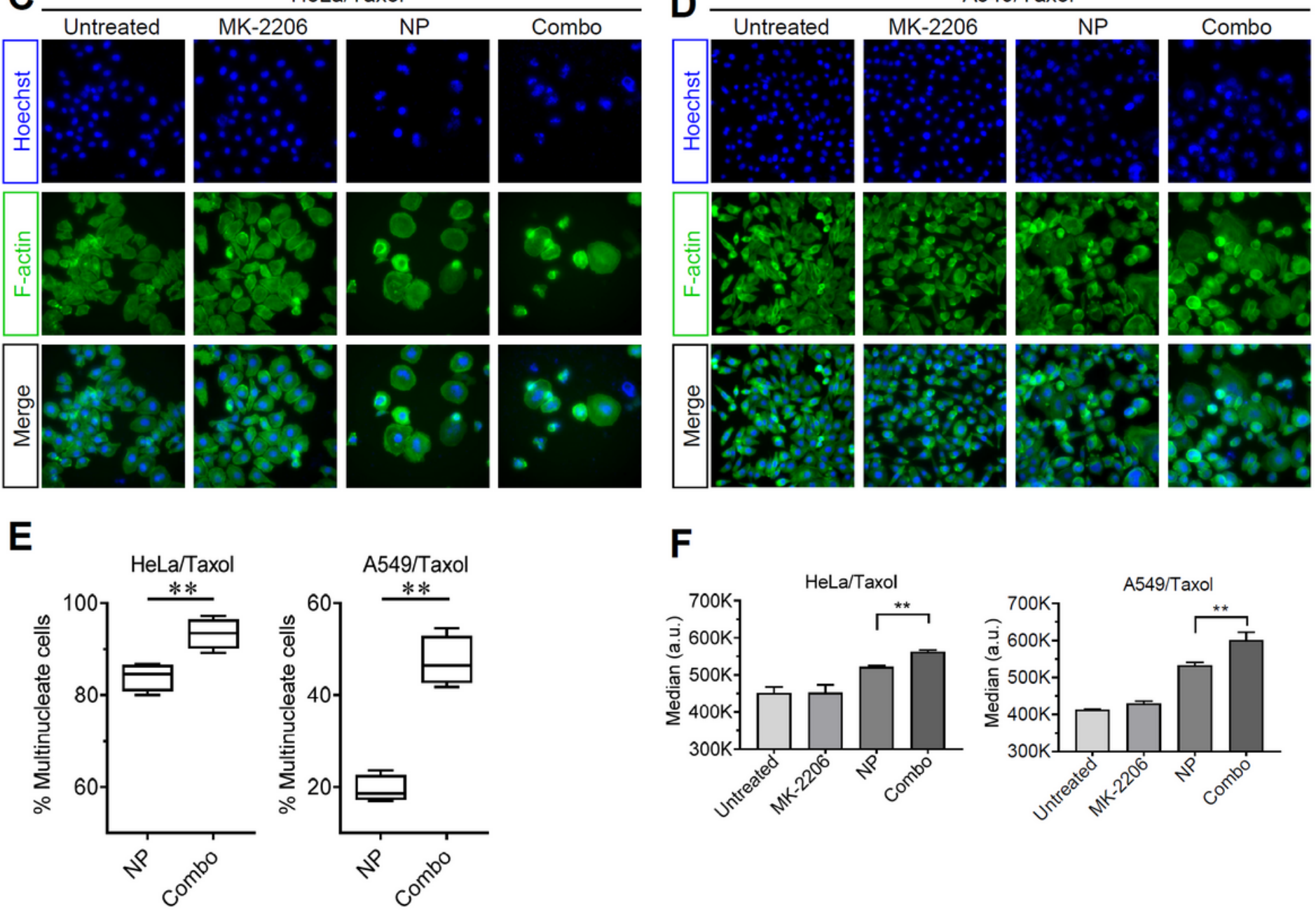

$\mathbf{F}$
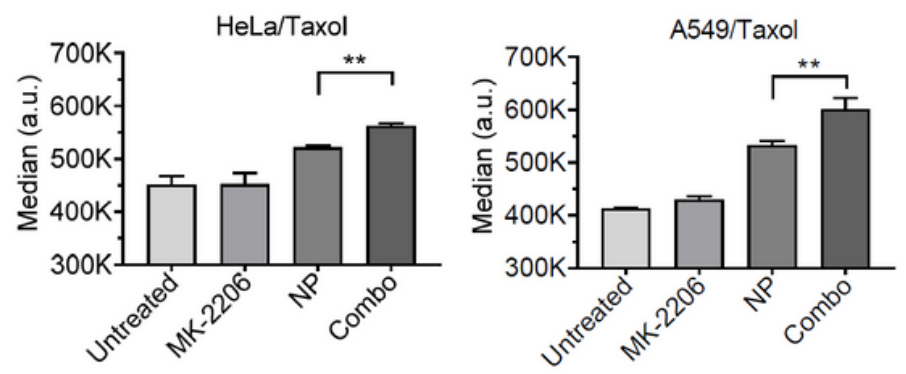

Figure 5

MK-2206 enhances the proportion of cell cycle arrest mediated by cabazitaxel-NPs. a, b Cell cycle profiles of HeLa/Taxol and A549/Taxol cells exposed to MK-2206 and/or cabazitaxel-NPs. c, d Staining with Hoechst and Alexa Fluor 488-labeled phalloidin reveals alternations in nuclear morphology and cytoplasmic volume in HeLa/Taxol cells (c) and A549/Taxol cells (d) after different treatments. e Box whisker plots indicate the fraction of the cells with abnormal mitoses (e.g., multinuclear defects) after exposure to cabazitaxel-NPs or the combination regimen. $f$ Forward scattering shows the heterogeneity in cell size after various treatments. The data are presented as the means \pm SD; $* \star p<0.01$. 

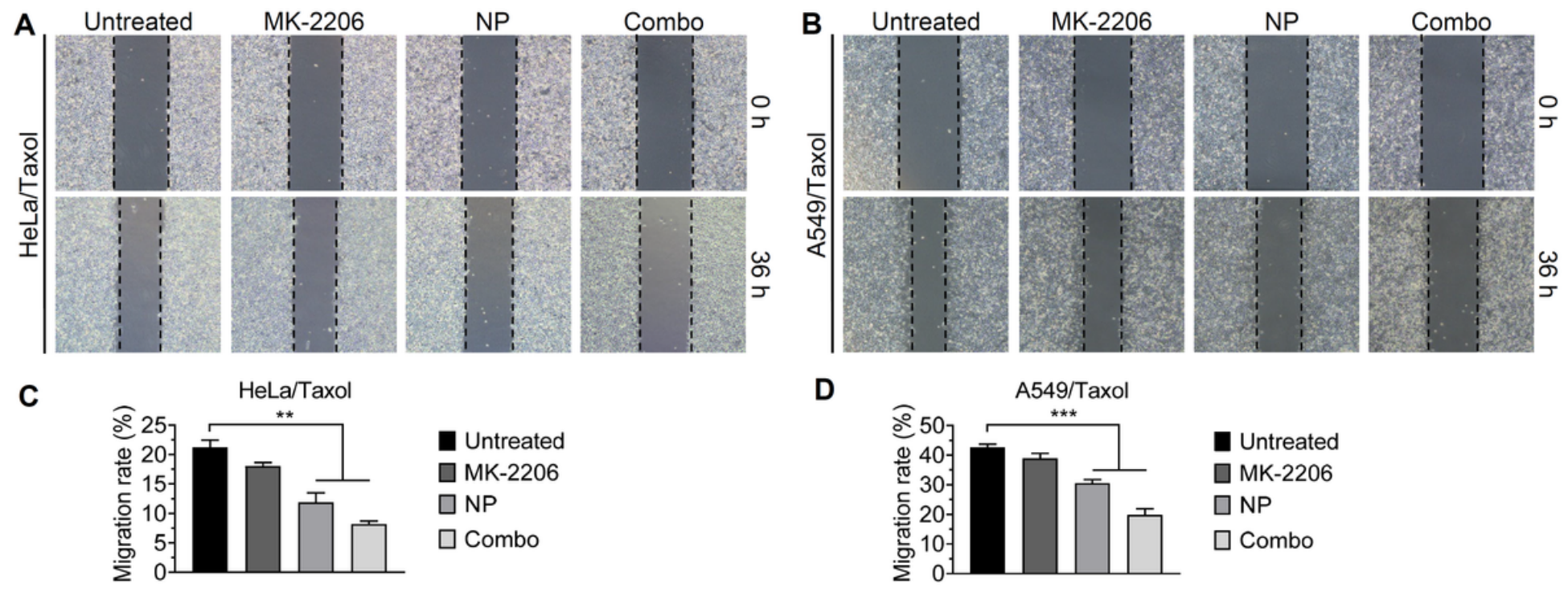

D
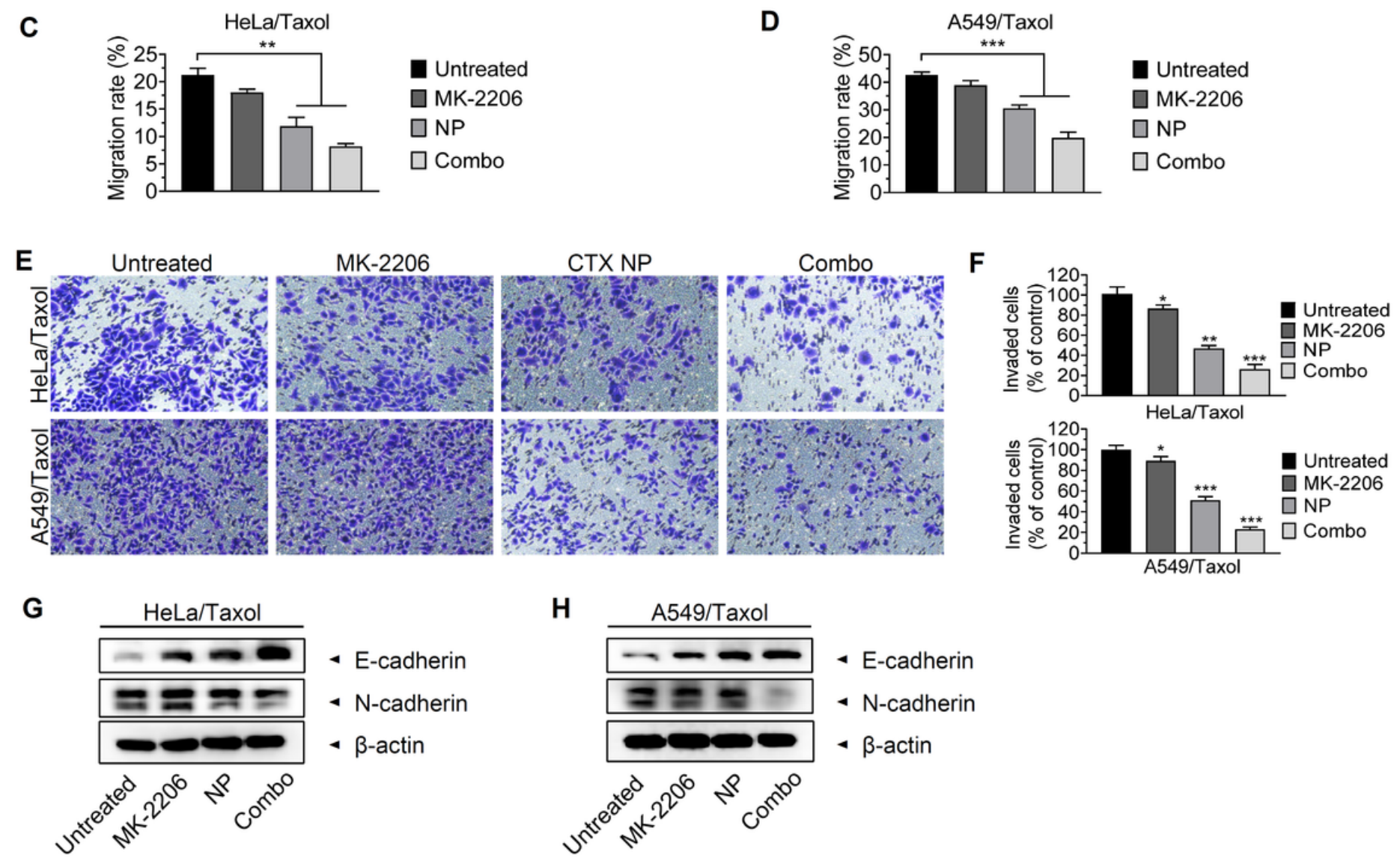

Figure 6

MK-2206 potentiates the anti-metastatic effect of cabazitaxel-NPs in resistant cancer cells. a, b Wound healing assay in HeLa/Taxol (a) and A549/Taxol (b) cells after treatment with subtoxic MK-2206 and/or cabazitaxel-NPs for $36 \mathrm{~h}$ (magnification, $100 \times$ ). c, d Quantitative analyses indicate the reduced cell motility of HeLa/Taxol (c) and A549/Taxol (d) cells after the treatments. e Representative images of invaded cells across the membrane after incubation with different drugs (magnification, $200 \times$ ). $f$ The invasive cells were quantified relative to the percentages of untreated cells. Upper, HeLa/Taxol cells; lower, A549/Taxol cells. g, $\mathrm{h}$ Western blot analysis of the expression levels of E-cadherin and $\mathrm{N}$-cadherin after exposure to drugs. The data are presented as the means $\pm S D ; * p<0.05$, $* \star p<0.01$, and ${ }^{\star \star \star} p<0.001$. 

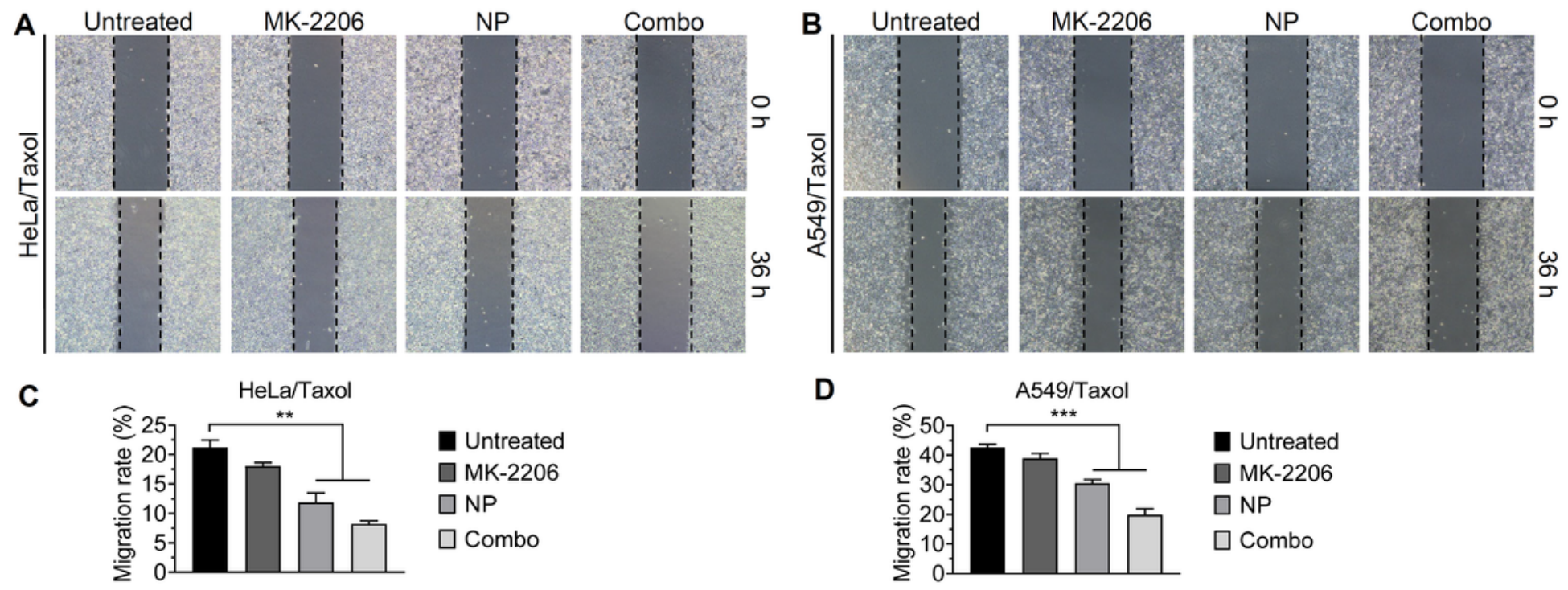

D
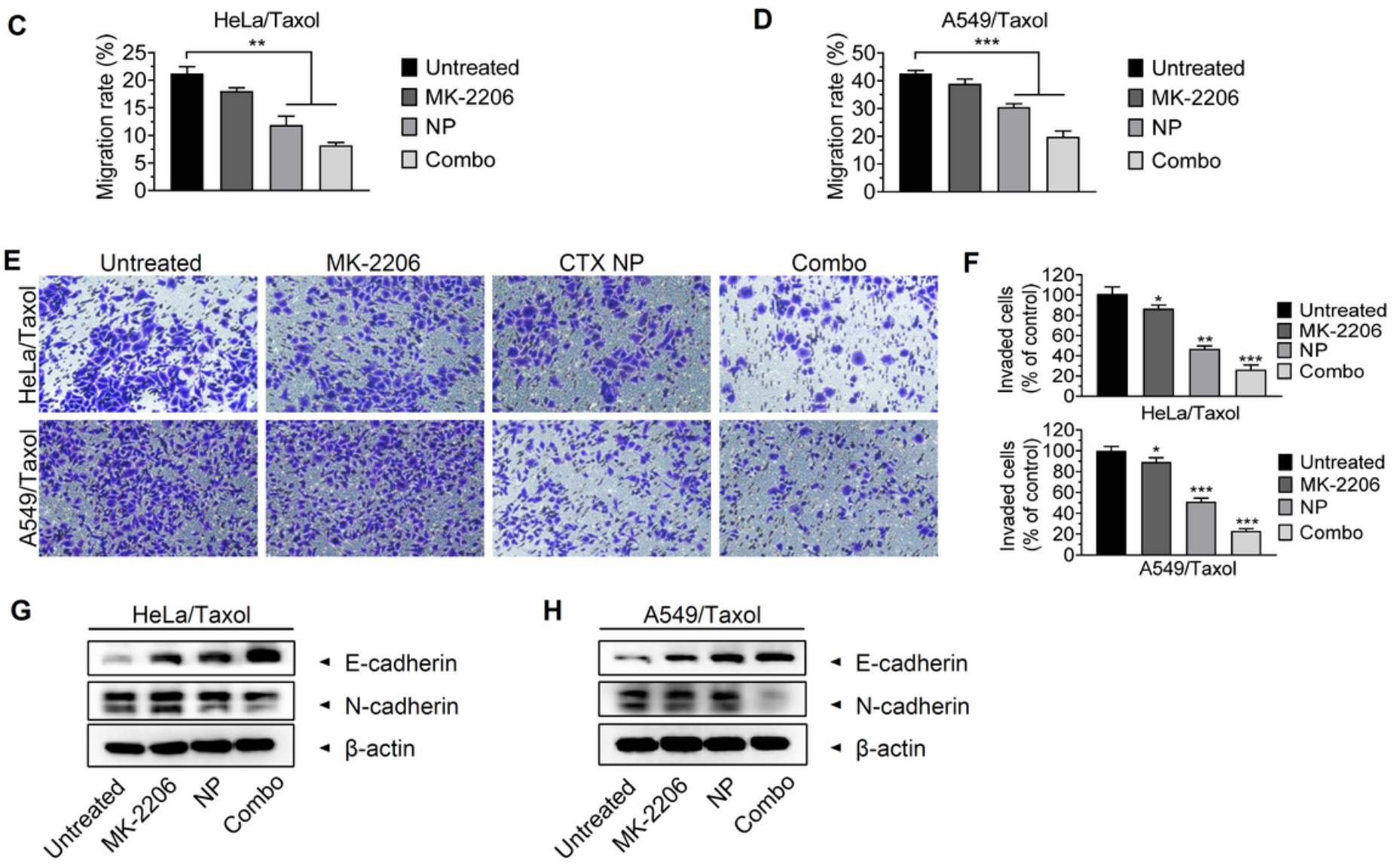

Figure 6

MK-2206 potentiates the anti-metastatic effect of cabazitaxel-NPs in resistant cancer cells. a, b Wound healing assay in HeLa/Taxol (a) and A549/Taxol (b) cells after treatment with subtoxic MK-2206 and/or cabazitaxel-NPs for $36 \mathrm{~h}$ (magnification, $100 \times$ ). c, d Quantitative analyses indicate the reduced cell motility of HeLa/Taxol (c) and A549/Taxol (d) cells after the treatments. e Representative images of invaded cells across the membrane after incubation with different drugs (magnification, $200 \times$ ). $f$ The invasive cells were quantified relative to the percentages of untreated cells. Upper, HeLa/Taxol cells; lower, A549/Taxol cells. g, $\mathrm{h}$ Western blot analysis of the expression levels of E-cadherin and $\mathrm{N}$-cadherin after exposure to drugs. The data are presented as the means $\pm S D ; * p<0.05$, $* \star p<0.01$, and ${ }^{\star \star \star} p<0.001$. 

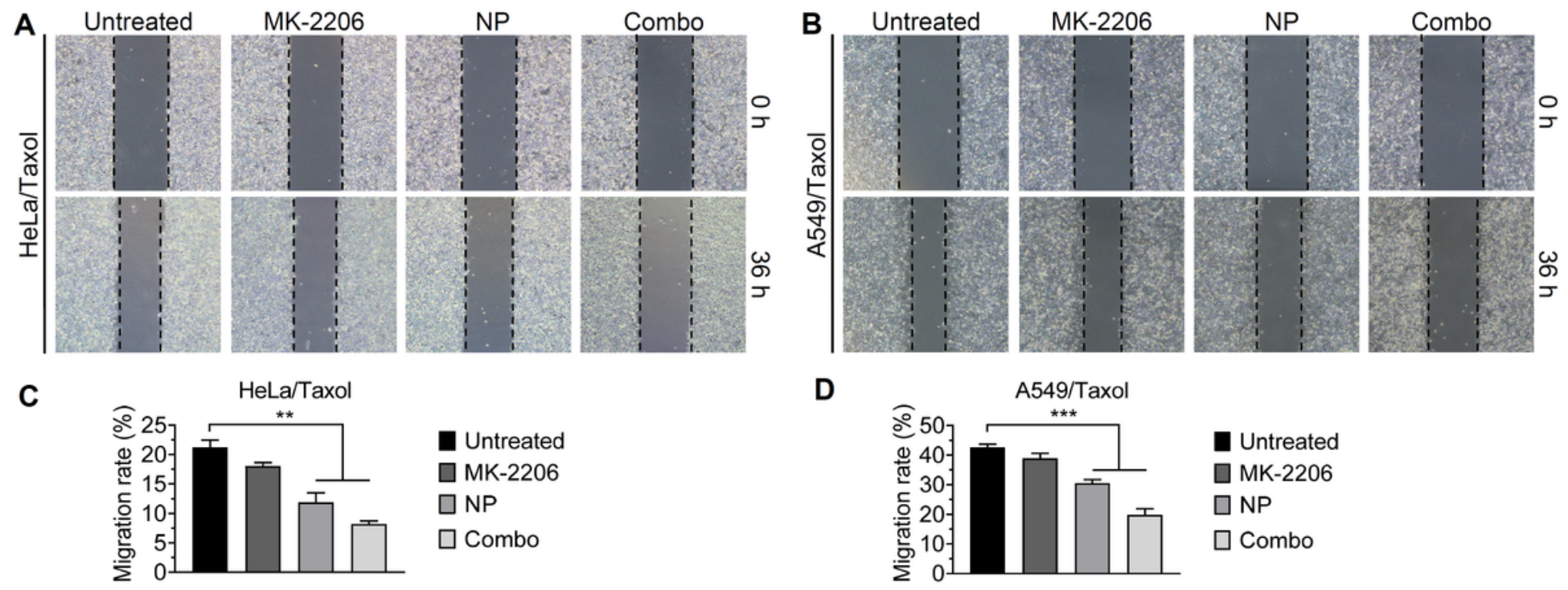

D
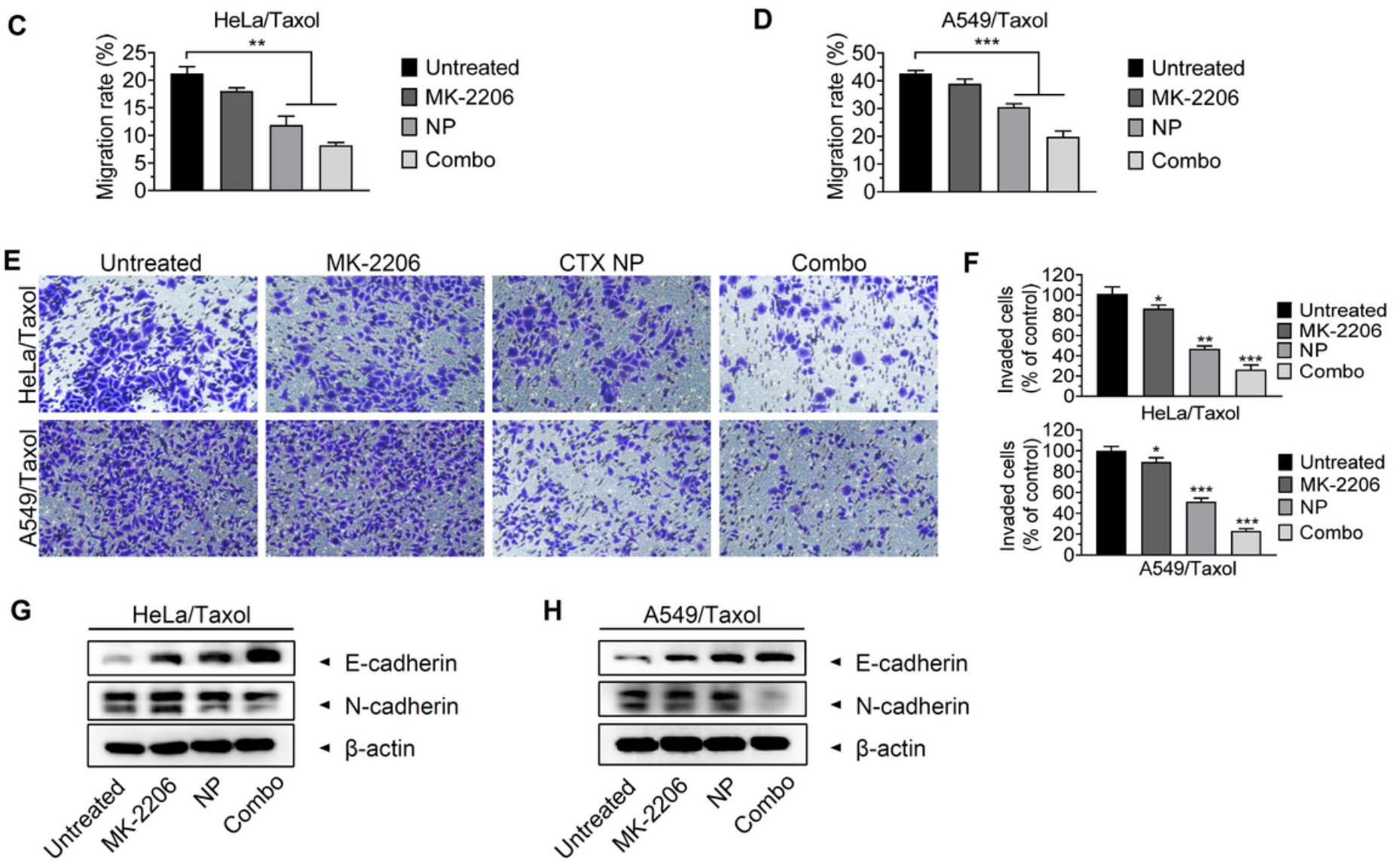

Figure 6

MK-2206 potentiates the anti-metastatic effect of cabazitaxel-NPs in resistant cancer cells. a, b Wound healing assay in HeLa/Taxol (a) and A549/Taxol (b) cells after treatment with subtoxic MK-2206 and/or cabazitaxel-NPs for $36 \mathrm{~h}$ (magnification, $100 \times$ ). c, d Quantitative analyses indicate the reduced cell motility of HeLa/Taxol (c) and A549/Taxol (d) cells after the treatments. e Representative images of invaded cells across the membrane after incubation with different drugs (magnification, $200 \times$ ). $f$ The invasive cells were quantified relative to the percentages of untreated cells. Upper, HeLa/Taxol cells; lower, A549/Taxol cells. g, $\mathrm{h}$ Western blot analysis of the expression levels of E-cadherin and $\mathrm{N}$-cadherin after exposure to drugs. The data are presented as the means $\pm S D ; * p<0.05$, $* \star p<0.01$, and ${ }^{\star \star \star} p<0.001$. 
A

HeLa/Taxol

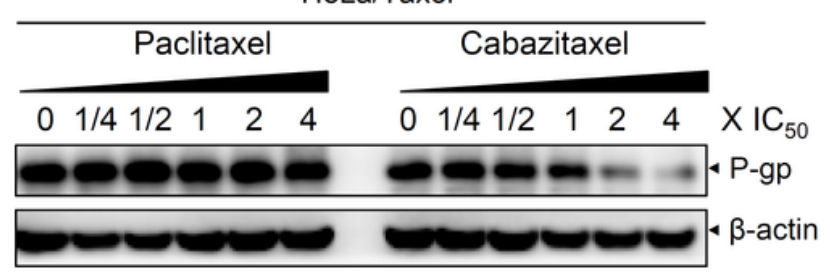

A549/Taxol

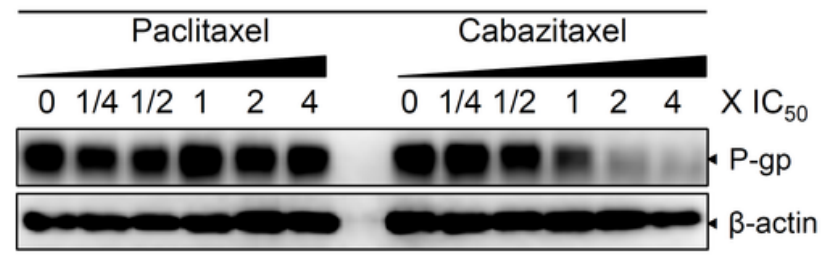

B

HeLa/Taxol

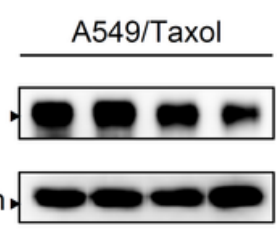

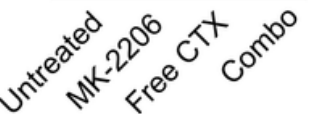

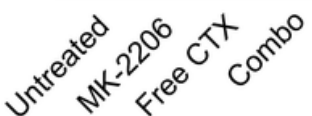

C HeLa/Taxol

A549/Taxol

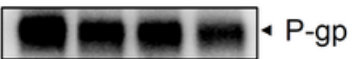

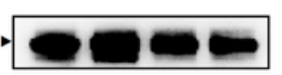

De- $\beta$-actin

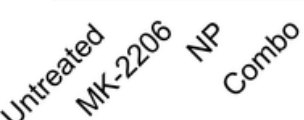

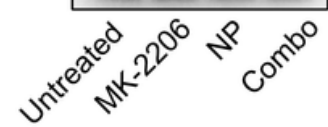

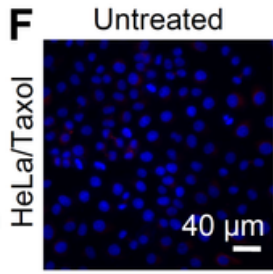
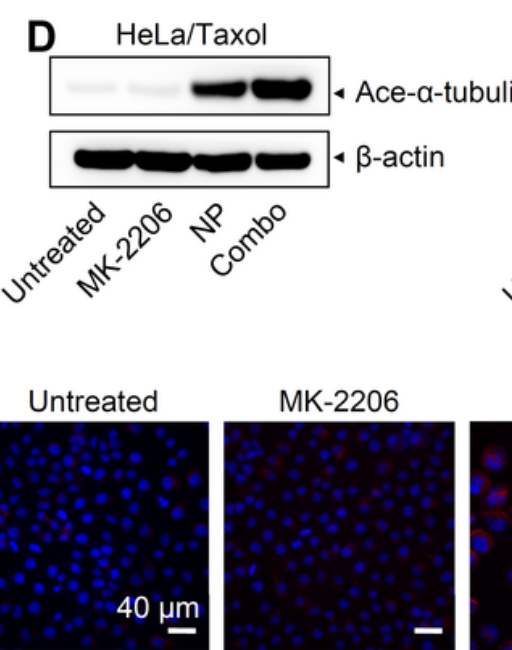

E A549/Taxo
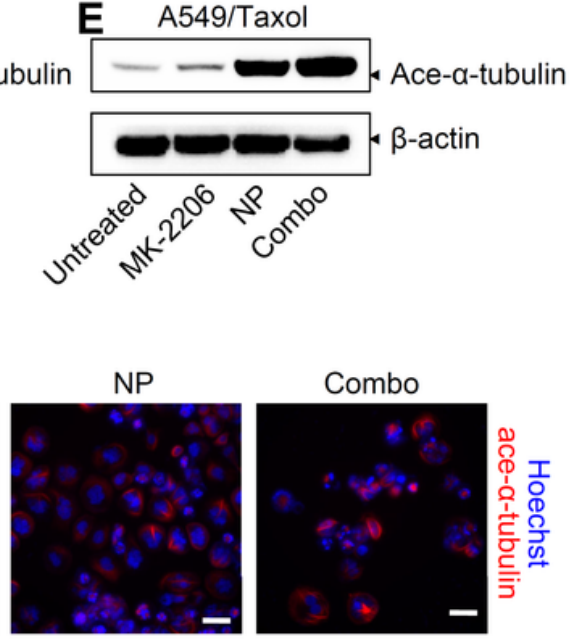

Combo

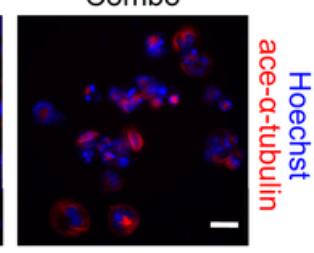

$40 \mu \mathrm{m}$
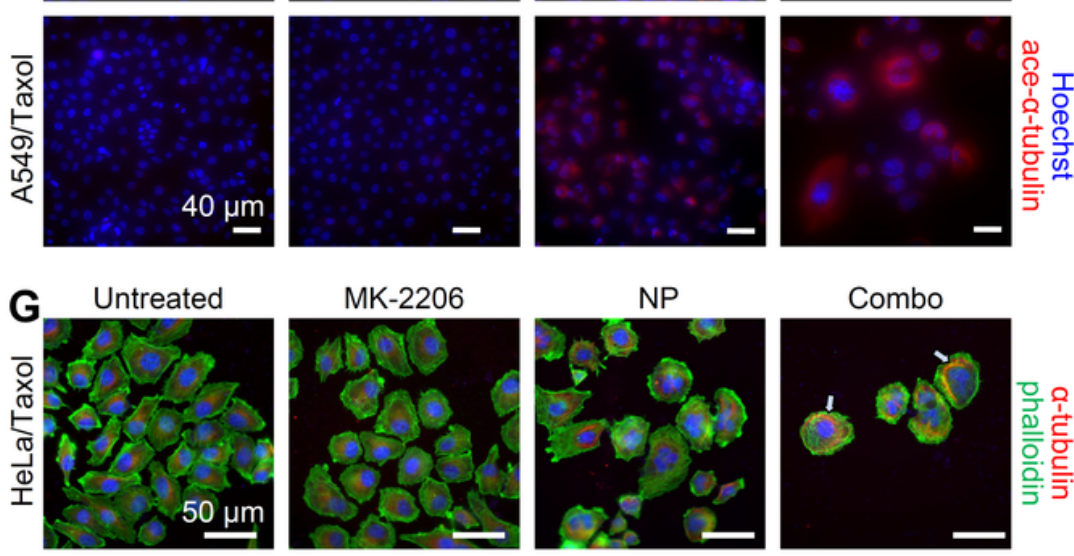

Combo
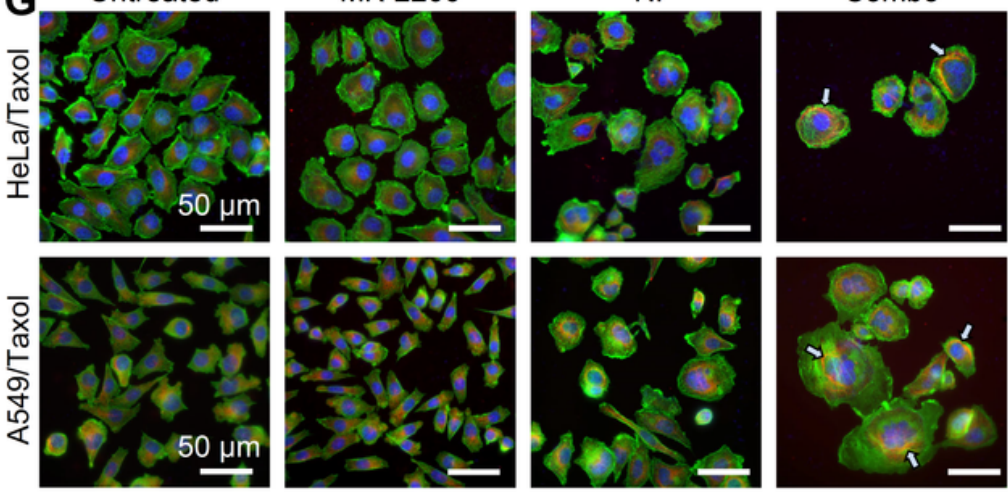

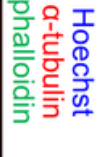

\section{Figure 7}

The combination of Akt inhibitor and cabazitaxel-NPs reduces P-gp and stabilizes microtubules in taxane-resistant cells. a Upon exposure to paclitaxel and cabazitaxel at different concentrations for $48 \mathrm{~h}$, cells were examined for P-gp expression. b The protein levels of P-gp decreased after HeLa/Taxol (left) and A549/Taxol (right) cells were treated with the indicated concentrations of cabazitaxel and/or their combinations with $3 \mu \mathrm{M}$ MK-2206. c Changes in P-gp protein levels in HeLa/Taxol (left) and A549/Taxol (right) cells after exposure to the NP-based combination. d, e Immunoblotting of acetyl-a-tubulin after treatment with DMSO, MK-2206 (3 $\mu \mathrm{M})$, cabazitaxel-NPs (50 nM), or the combination regimen for $48 \mathrm{~h}$. $\mathrm{f}$ Immunofluorescence staining of acetyl-a-tubulin (red) in taxane-resistant cell lines. Scale bars, $40 \mu \mathrm{m}$. $\mathrm{g}$ Immunofluorescence staining in cells. Red: a-tubulin; green: F-actin; blue: Hoechst (nuclei). Scale bars, 50 $\mu \mathrm{m}$. 
A

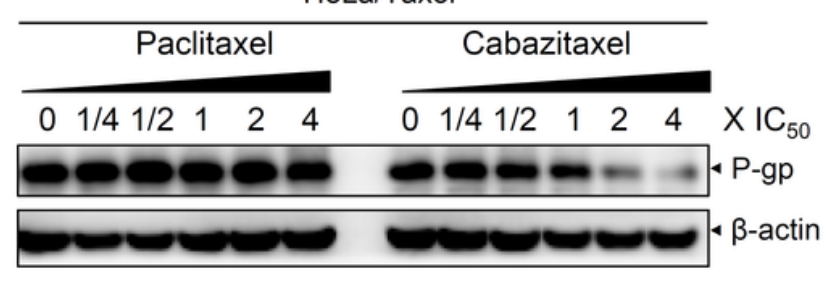

A549/Taxol

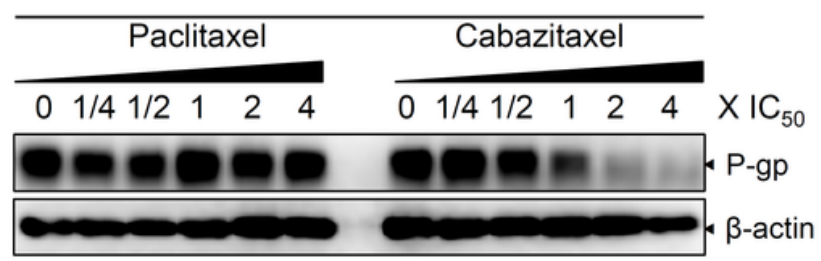

B

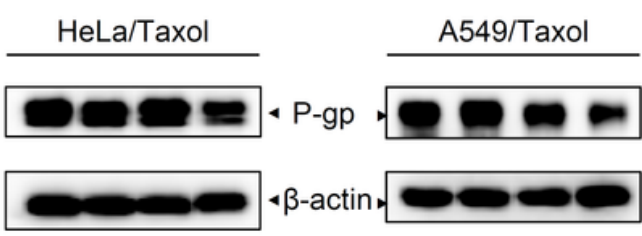

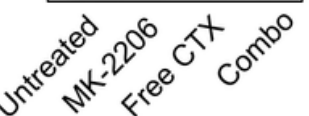

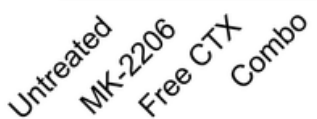

C HeLa/Taxol

A549/Taxol

Q60 P-gp

- $\beta$-actin

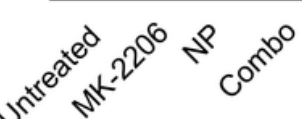

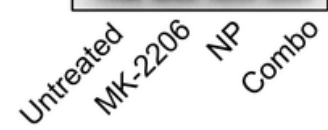

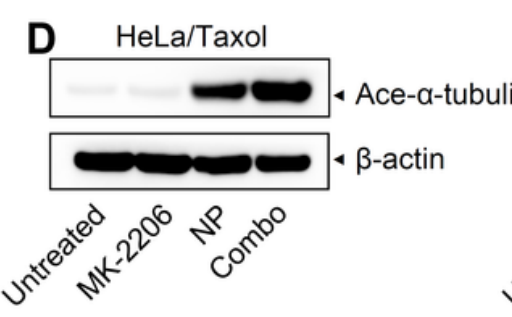

E A549/Taxol

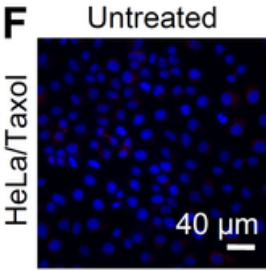

MK-2206
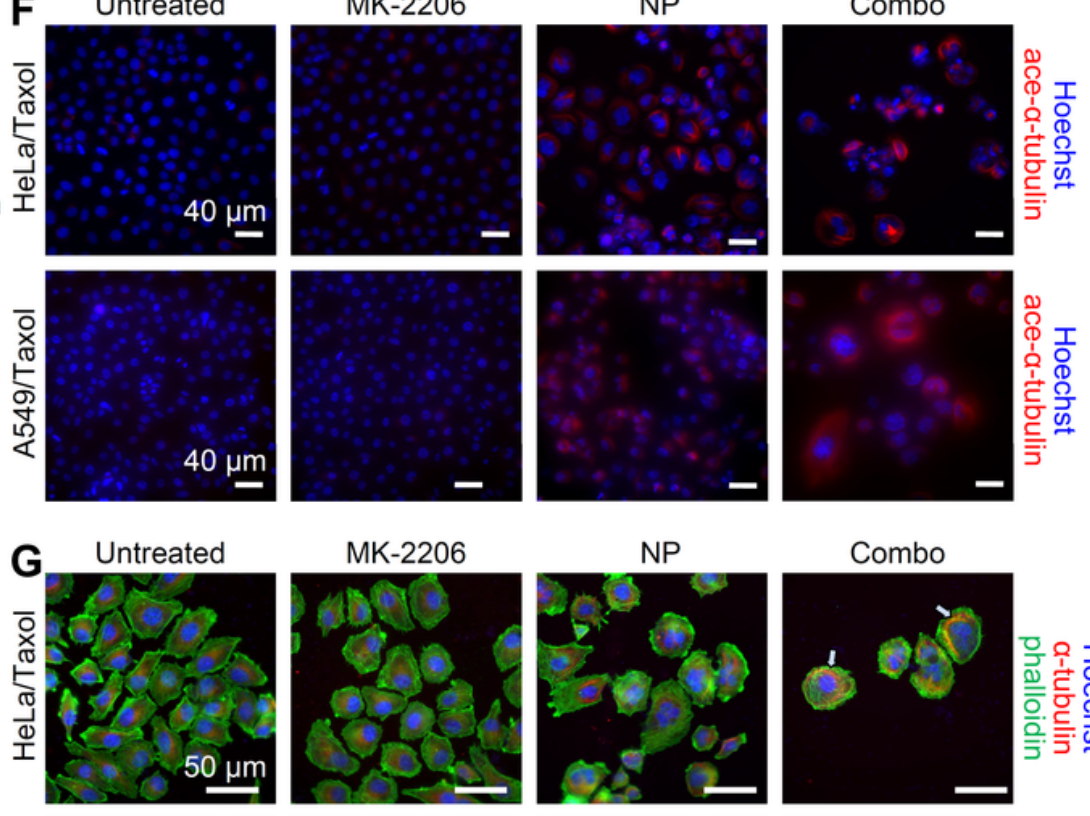

Combo
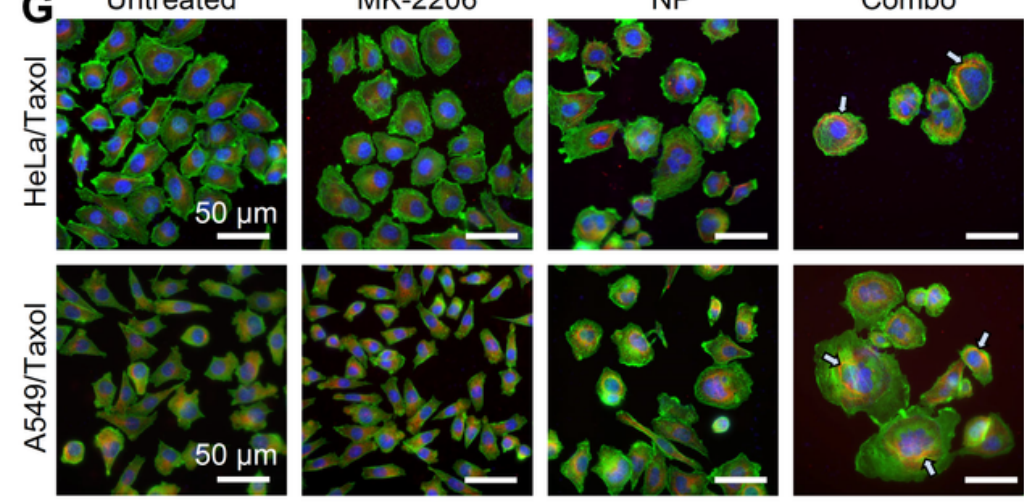

Figure 7

The combination of Akt inhibitor and cabazitaxel-NPs reduces P-gp and stabilizes microtubules in taxane-resistant cells. a Upon exposure to paclitaxel and cabazitaxel at different concentrations for $48 \mathrm{~h}$, cells were examined for P-gp expression. b The protein levels of P-gp decreased after HeLa/Taxol (left) and A549/Taxol (right) cells were treated with the indicated concentrations of cabazitaxel and/or their combinations with $3 \mu \mathrm{M}$ MK-2206. c Changes in P-gp protein levels in HeLa/Taxol (left) and A549/Taxol (right) cells after exposure to the NP-based combination. d, e Immunoblotting of acetyl-a-tubulin after treatment with DMSO, MK-2206 $(3 \mu \mathrm{M})$, cabazitaxel-NPs $(50 \mathrm{nM})$, or the combination regimen for $48 \mathrm{~h}$. $f$ Immunofluorescence staining of acetyl-a-tubulin (red) in taxane-resistant cell lines. Scale bars, $40 \mu \mathrm{m} . \mathrm{g}$ Immunofluorescence staining in cells. Red: a-tubulin; green: F-actin; blue: Hoechst (nuclei). Scale bars, 50 $\mu \mathrm{m}$. 
A

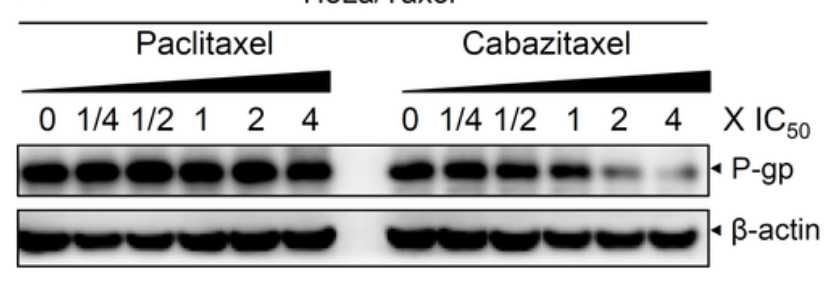

A549/Taxol

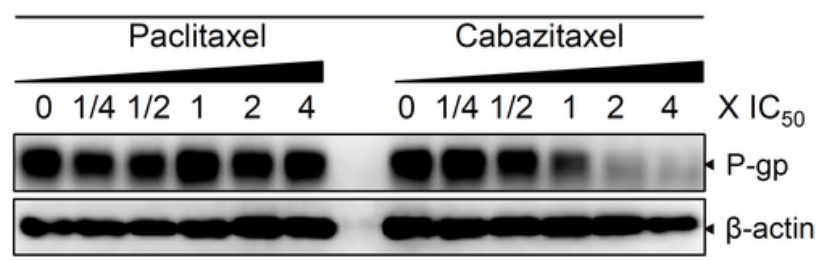

B

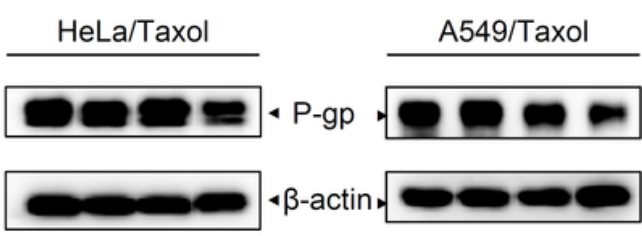

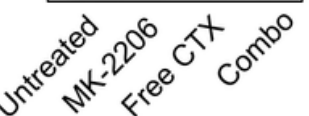

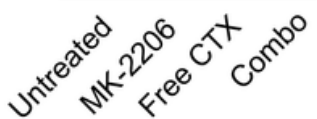

C HeLa/Taxol

A549/Taxol

Q60 P-gp

De- $\beta$-actin

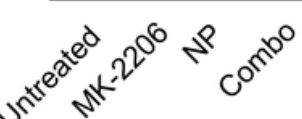

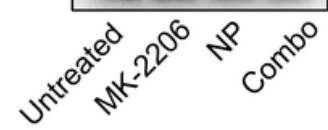

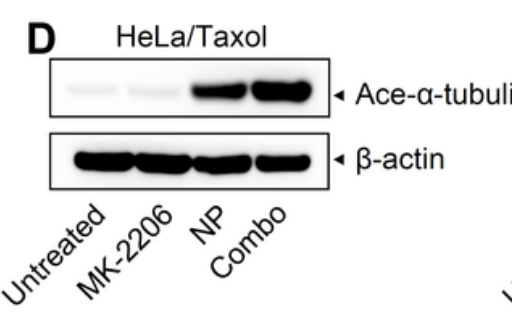

E A549/Taxol

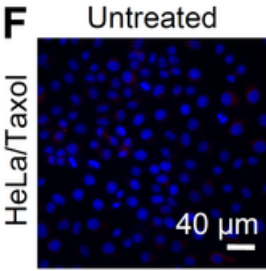

MK-2206
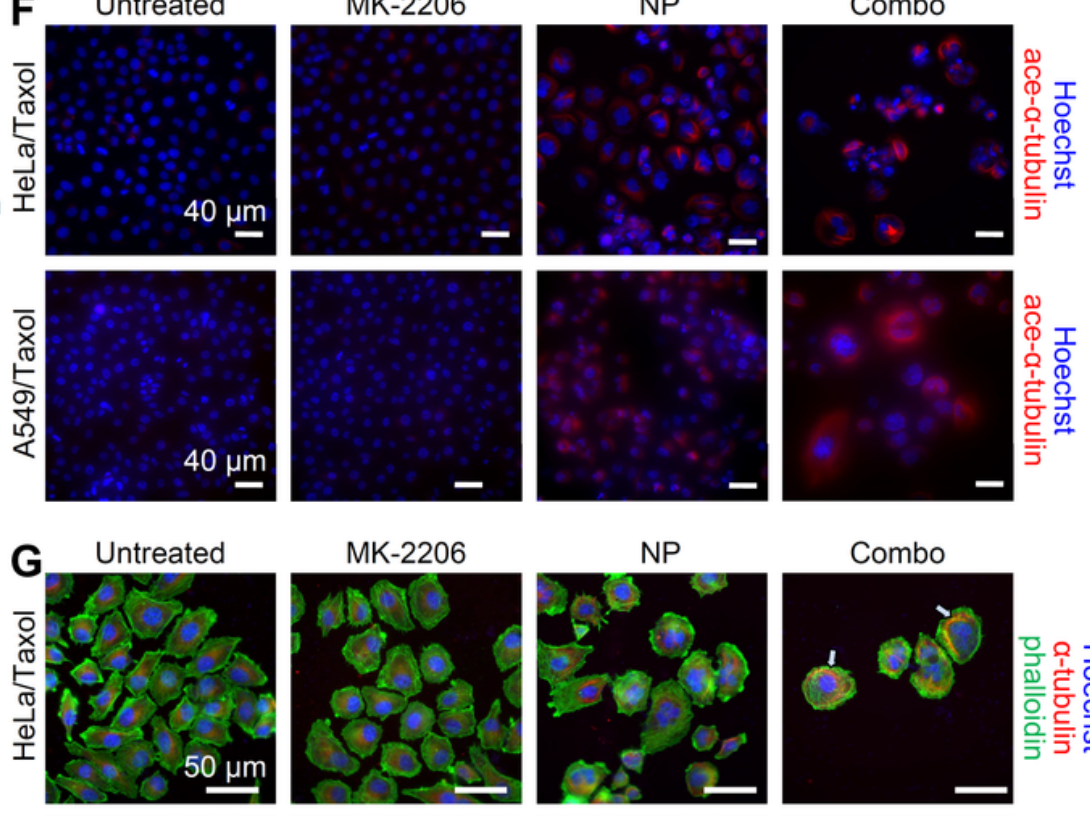

Combo
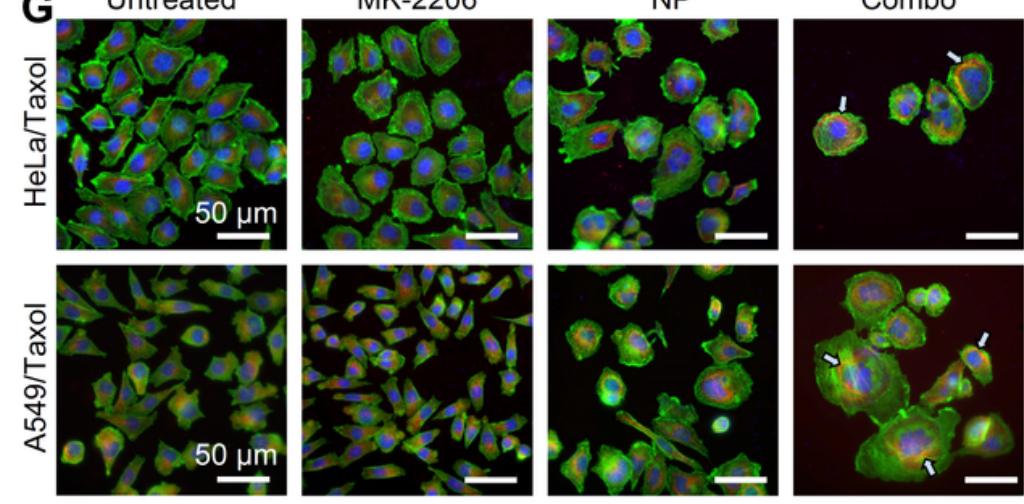

Figure 7

The combination of Akt inhibitor and cabazitaxel-NPs reduces P-gp and stabilizes microtubules in taxane-resistant cells. a Upon exposure to paclitaxel and cabazitaxel at different concentrations for $48 \mathrm{~h}$, cells were examined for P-gp expression. b The protein levels of P-gp decreased after HeLa/Taxol (left) and A549/Taxol (right) cells were treated with the indicated concentrations of cabazitaxel and/or their combinations with $3 \mu \mathrm{M}$ MK-2206. c Changes in P-gp protein levels in HeLa/Taxol (left) and A549/Taxol (right) cells after exposure to the NP-based combination. d, e Immunoblotting of acetyl-a-tubulin after treatment with DMSO, MK-2206 $(3 \mu \mathrm{M})$, cabazitaxel-NPs $(50 \mathrm{nM})$, or the combination regimen for $48 \mathrm{~h}$. $f$ Immunofluorescence staining of acetyl-a-tubulin (red) in taxane-resistant cell lines. Scale bars, $40 \mu \mathrm{m} . \mathrm{g}$ Immunofluorescence staining in cells. Red: a-tubulin; green: F-actin; blue: Hoechst (nuclei). Scale bars, 50 $\mu \mathrm{m}$. 


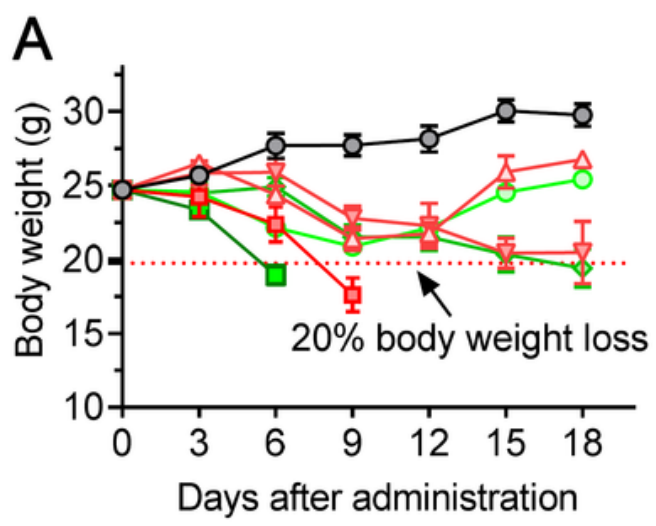

\begin{tabular}{|c|c|c|}
\hline Drug & Dose (i.v.) & $\begin{array}{l}\text { Survival at } \\
\text { endpoints }\end{array}$ \\
\hline Control & Saline & $3 / 3$ \\
\hline \multirow{3}{*}{ Free CTX } & $10 \mathrm{mg} / \mathrm{kg}$ (q036d) & $3 / 3$ \\
\hline & $15 \mathrm{mg} / \mathrm{kg}$ (q036d) & $2 / 3$ \\
\hline & $20 \mathrm{mg} / \mathrm{kg}$ (q036d) & $0 / 3$ \\
\hline \multirow{3}{*}{ NP } & $40 \mathrm{mg} / \mathrm{kg}$ (q036d) & $3 / 3$ \\
\hline & $50 \mathrm{mg} / \mathrm{kg}$ (q036d) & $1 / 3$ \\
\hline & $60 \mathrm{mg} / \mathrm{kg}$ (q036d) & $0 / 3$ \\
\hline
\end{tabular}
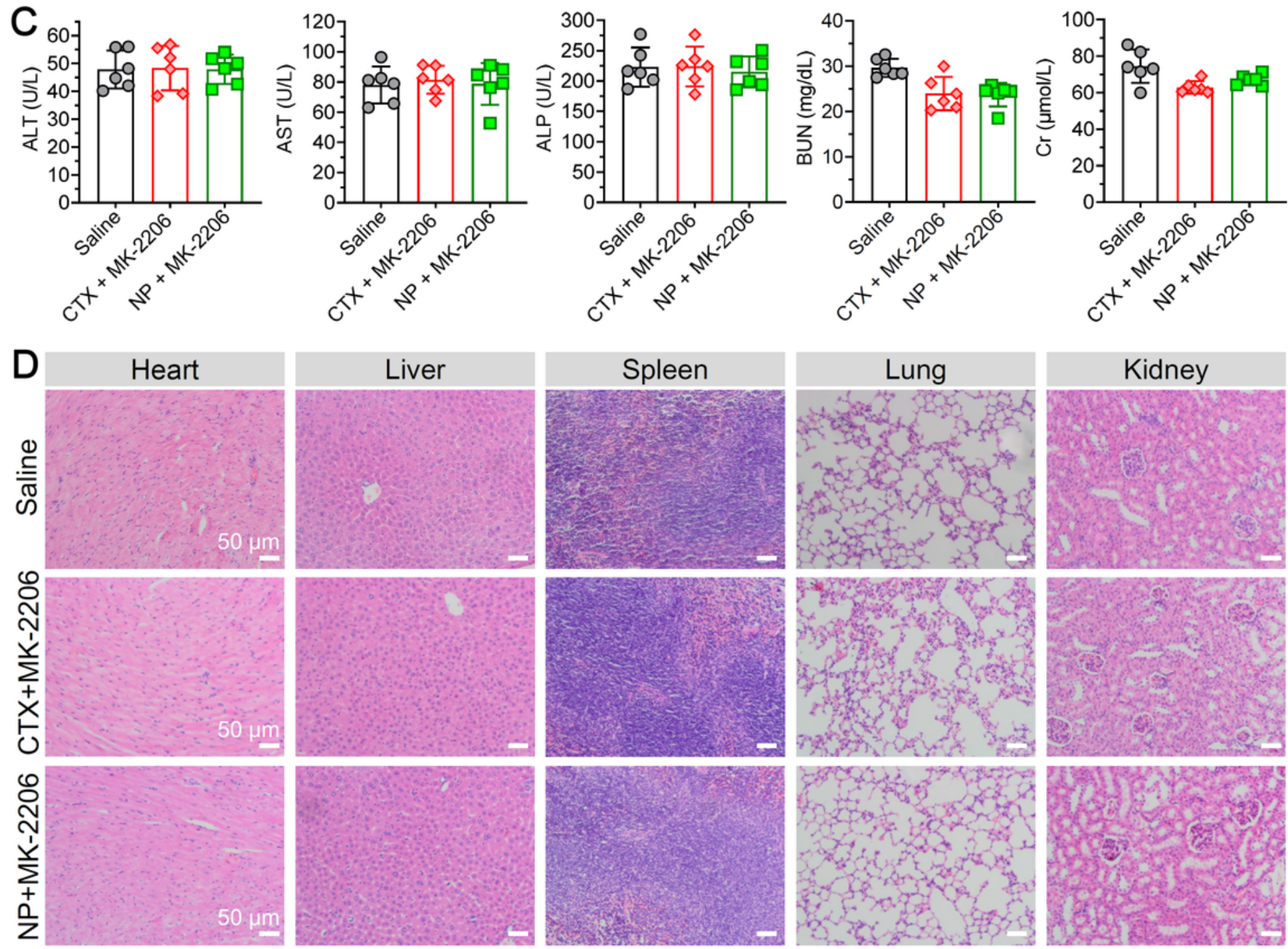

\section{Figure 8}

Safety evaluation of cabazitaxel nanoparticles when coadministered with MK-2206 in ICR mice. a Body weight variations were recorded after treatment with drugs. b Animal survival in each group at the endpoints. c Serological parameters at day 1 post-injection in each group. The data are presented as the means $\pm S D(n=6)$. $d$ Tissue histology from the mice with different treatments. Representative images were selected from each group $(n=6)$. Scale bars, $50 \mu \mathrm{m}$. 


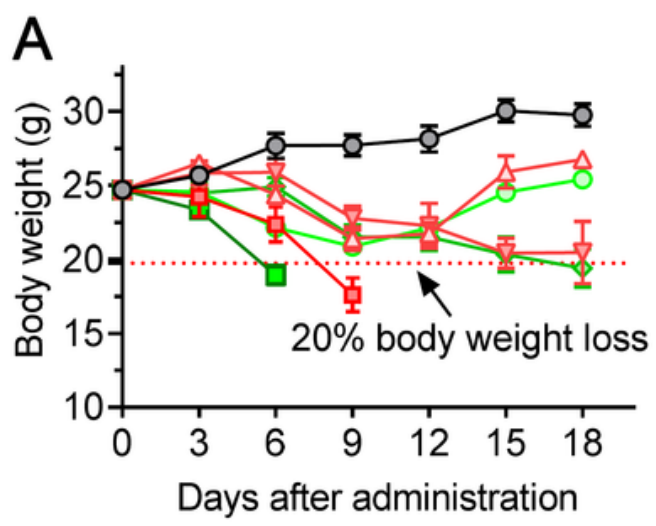

\begin{tabular}{|c|c|c|}
\hline Drug & Dose (i.v.) & $\begin{array}{l}\text { Survival at } \\
\text { endpoints }\end{array}$ \\
\hline Control & Saline & $3 / 3$ \\
\hline \multirow{3}{*}{ Free CTX } & $10 \mathrm{mg} / \mathrm{kg}$ (q036d) & $3 / 3$ \\
\hline & $15 \mathrm{mg} / \mathrm{kg}$ (q036d) & $2 / 3$ \\
\hline & $20 \mathrm{mg} / \mathrm{kg}$ (q036d) & $0 / 3$ \\
\hline \multirow{3}{*}{ NP } & $40 \mathrm{mg} / \mathrm{kg}$ (q036d) & $3 / 3$ \\
\hline & $50 \mathrm{mg} / \mathrm{kg}$ (q036d) & $1 / 3$ \\
\hline & $60 \mathrm{mg} / \mathrm{kg}$ (q036d) & $0 / 3$ \\
\hline
\end{tabular}
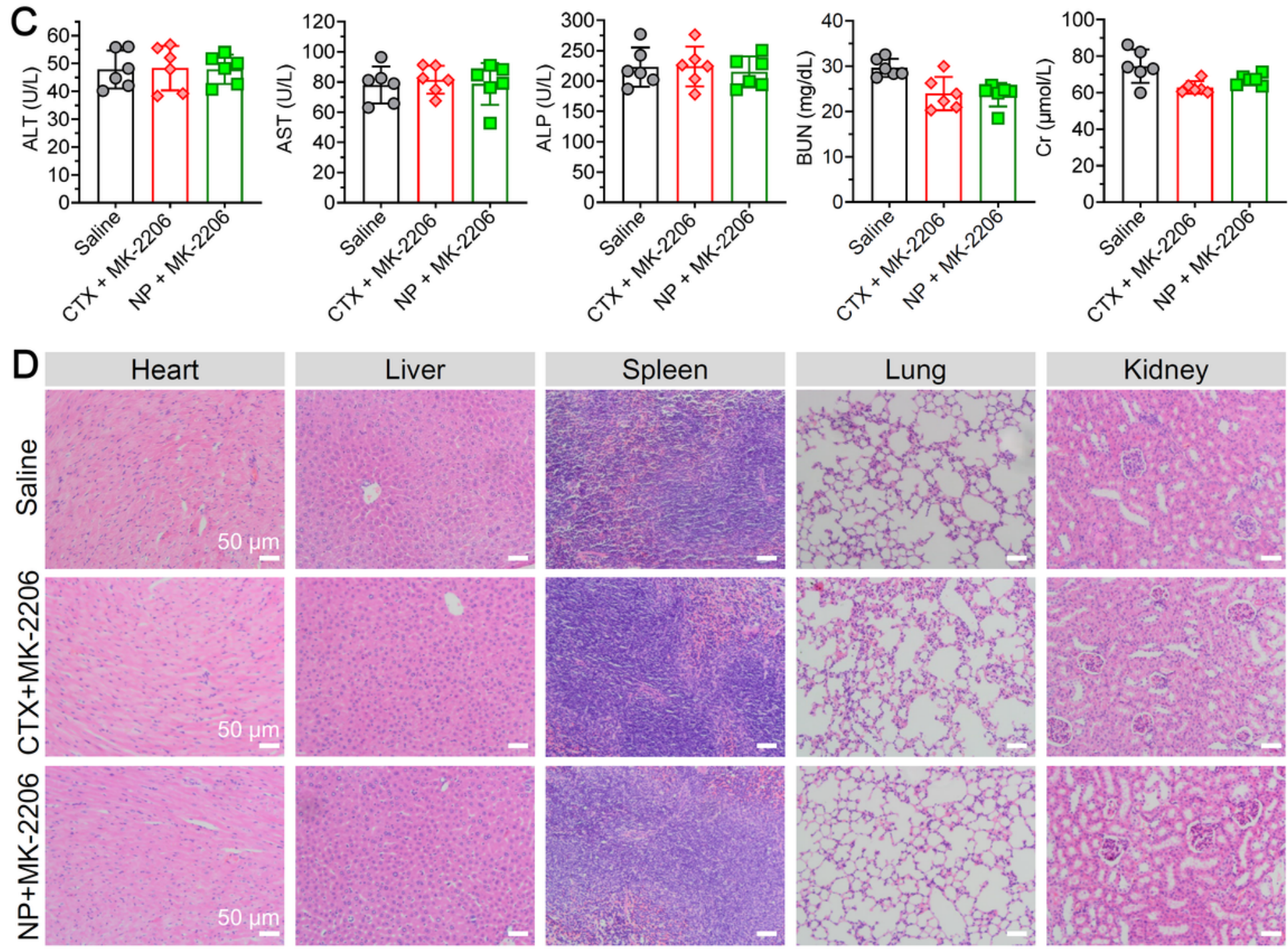

\section{Figure 8}

Safety evaluation of cabazitaxel nanoparticles when coadministered with MK-2206 in ICR mice. a Body weight variations were recorded after treatment with drugs. b Animal survival in each group at the endpoints. c Serological parameters at day 1 post-injection in each group. The data are presented as the means $\pm S D(n=6)$. $d$ Tissue histology from the mice with different treatments. Representative images were selected from each group $(n=6)$. Scale bars, $50 \mu \mathrm{m}$. 


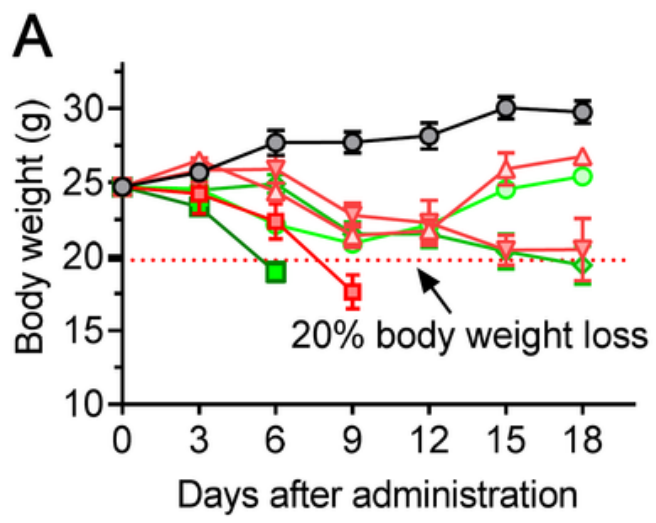

\begin{tabular}{|c|c|c|}
\hline Drug & Dose (i.v.) & $\begin{array}{l}\text { Survival at } \\
\text { endpoints }\end{array}$ \\
\hline Control & Saline & $3 / 3$ \\
\hline \multirow{3}{*}{ Free CTX } & $10 \mathrm{mg} / \mathrm{kg}(\mathrm{q} 036 \mathrm{~d})$ & $3 / 3$ \\
\hline & $15 \mathrm{mg} / \mathrm{kg}(\mathrm{q} 036 \mathrm{~d})$ & $2 / 3$ \\
\hline & $20 \mathrm{mg} / \mathrm{kg}$ (q036d) & $0 / 3$ \\
\hline \multirow{3}{*}{ NP } & $40 \mathrm{mg} / \mathrm{kg}$ (q036d) & $3 / 3$ \\
\hline & $50 \mathrm{mg} / \mathrm{kg}$ (q036d) & $1 / 3$ \\
\hline & $60 \mathrm{mg} / \mathrm{kg}(\mathrm{q} 036 \mathrm{~d})$ & $0 / 3$ \\
\hline
\end{tabular}
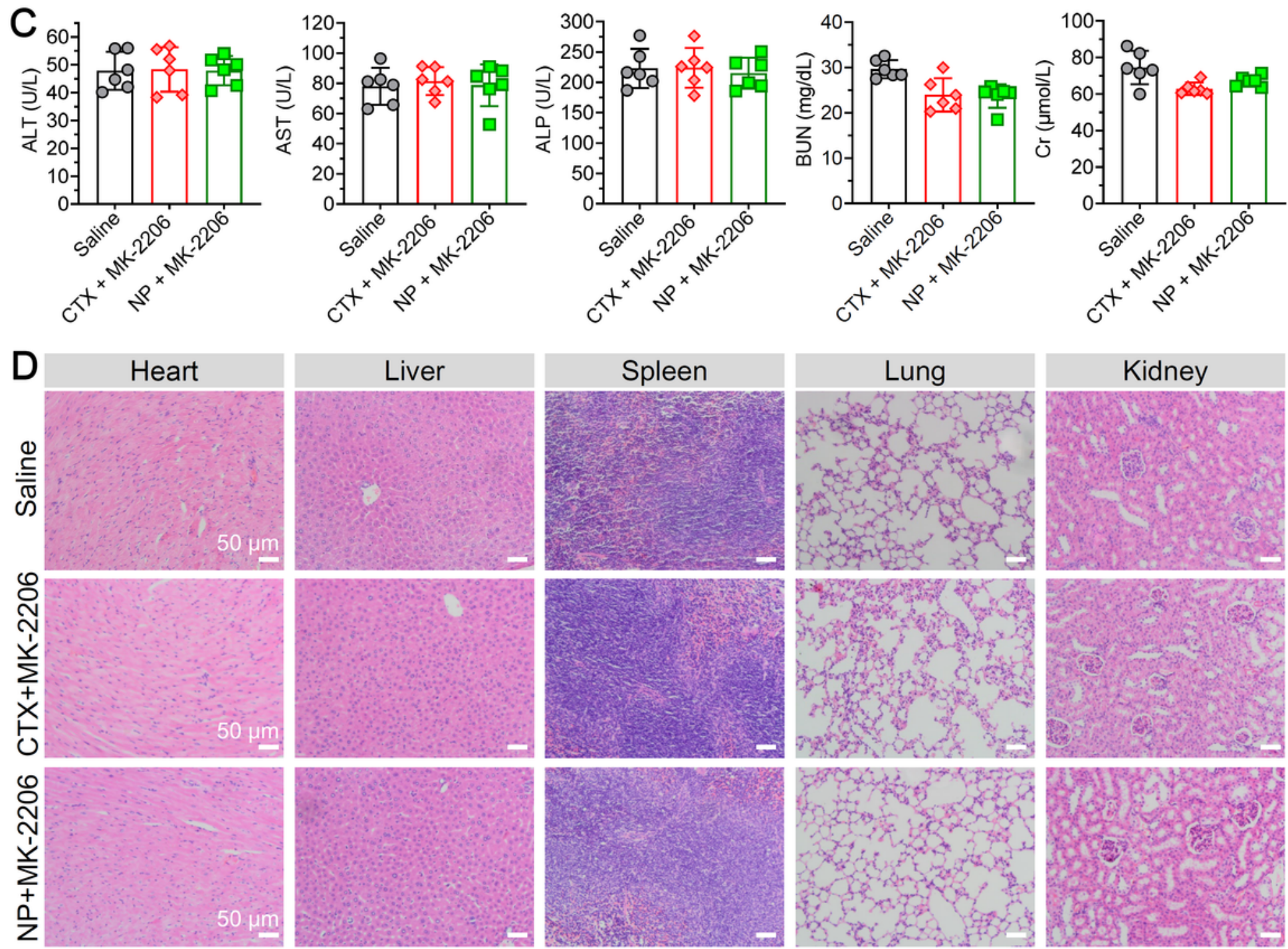

\section{Figure 8}

Safety evaluation of cabazitaxel nanoparticles when coadministered with MK-2206 in ICR mice. a Body weight variations were recorded after treatment with drugs. b Animal survival in each group at the endpoints. c Serological parameters at day 1 post-injection in each group. The data are presented as the means $\pm S D(n=6)$. $d$ Tissue histology from the mice with different treatments. Representative images were selected from each group $(n=6)$. Scale bars, $50 \mu \mathrm{m}$. 

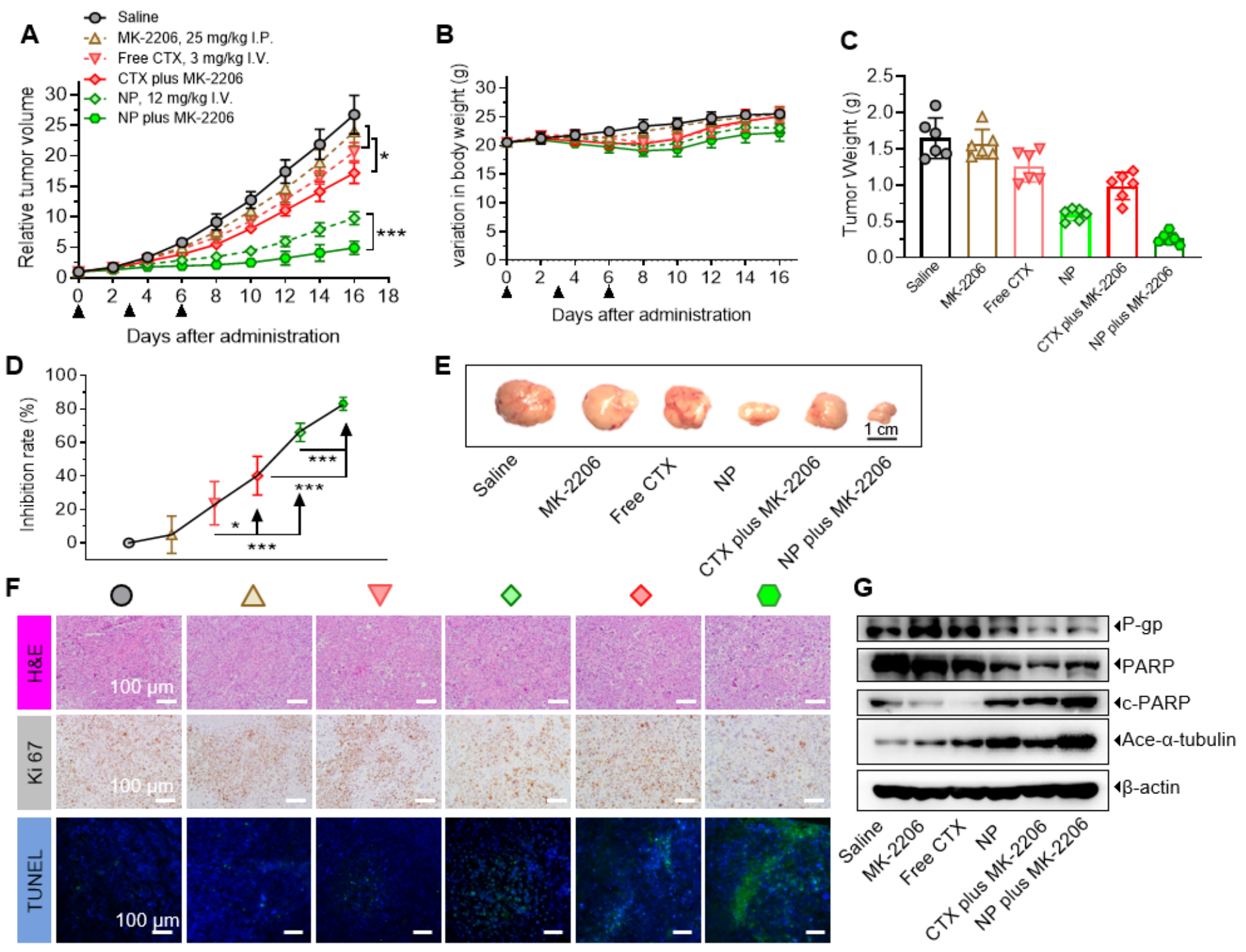

Figure 9

The combination of cabazitaxel-NPs and MK-2206 decreases tumor growth in HeLa/Taxol-bearing mice. a Tumor size changes during the treatments $(n=6)$. b Weight variations over time after various treatments $(n=6)$. Arrows indicate injection days. * indicates $p<0.05$, while $* \star \star$ indicates $p<0.001$ as determined by one-way ANOVA. c Tumor weights of each group at the endpoints. $d$ The tumor growth inhibition rate of each group was calculated according to tumor weights. e Representative images of tumors excised from different groups. Scale bar, $1 \mathrm{~cm}$. f Representative photographs of H\&E staining (upper), Ki-67 assay (middle), and TUNEL (lower) assay for tumor sections from different treatment groups at the endpoint. g Western blot results of P-gp, PARP, c-PARP, and acetyl-a-tubulin in isolated tumors. 

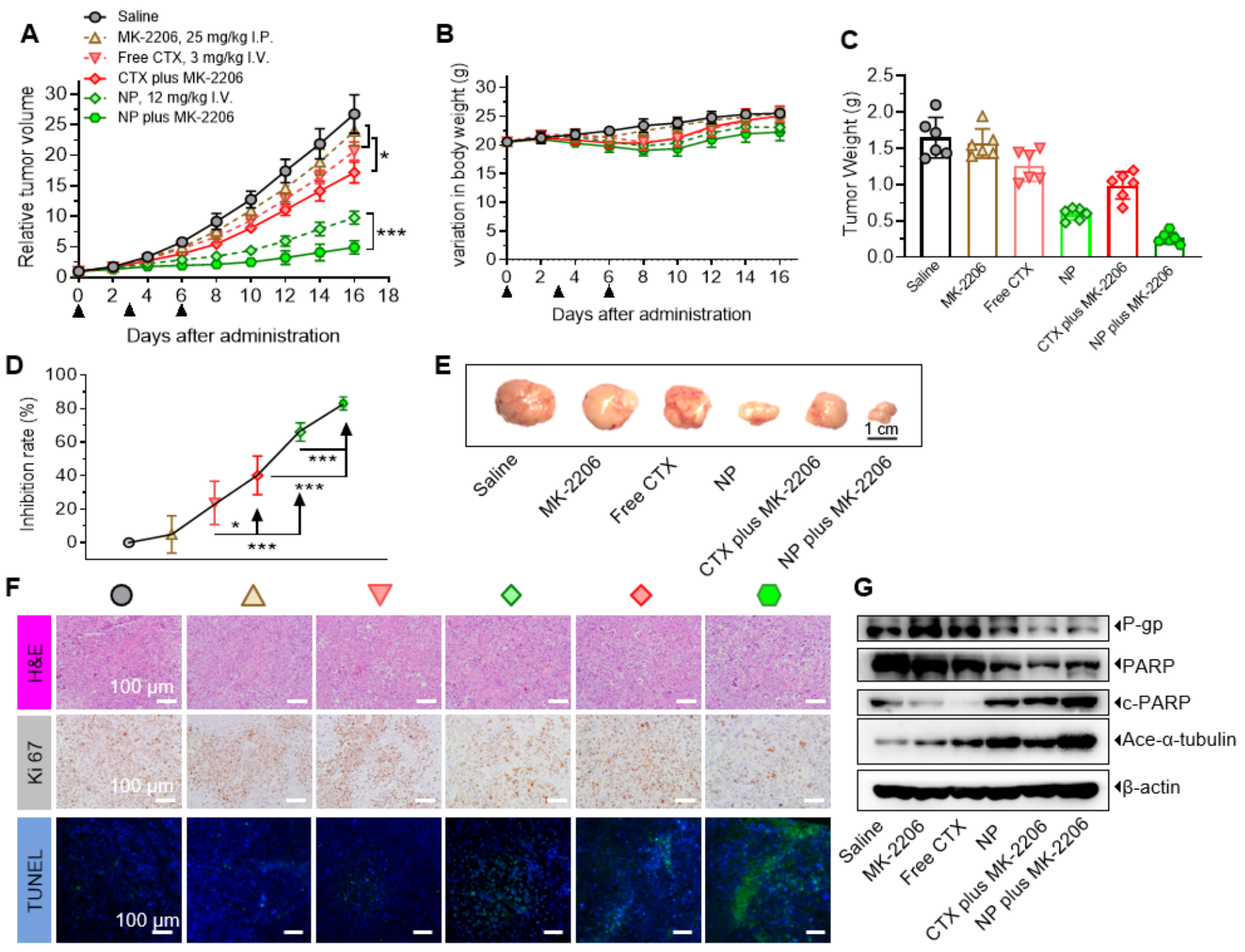

Figure 9

The combination of cabazitaxel-NPs and MK-2206 decreases tumor growth in HeLa/Taxol-bearing mice. a Tumor size changes during the treatments $(n=6)$. b Weight variations over time after various treatments $(n=6)$. Arrows indicate injection days. * indicates $p<0.05$, while $* \star \star$ indicates $p<0.001$ as determined by one-way ANOVA. c Tumor weights of each group at the endpoints. $d$ The tumor growth inhibition rate of each group was calculated according to tumor weights. e Representative images of tumors excised from different groups. Scale bar, $1 \mathrm{~cm}$. f Representative photographs of H\&E staining (upper), Ki-67 assay (middle), and TUNEL (lower) assay for tumor sections from different treatment groups at the endpoint. g Western blot results of P-gp, PARP, c-PARP, and acetyl-a-tubulin in isolated tumors. 

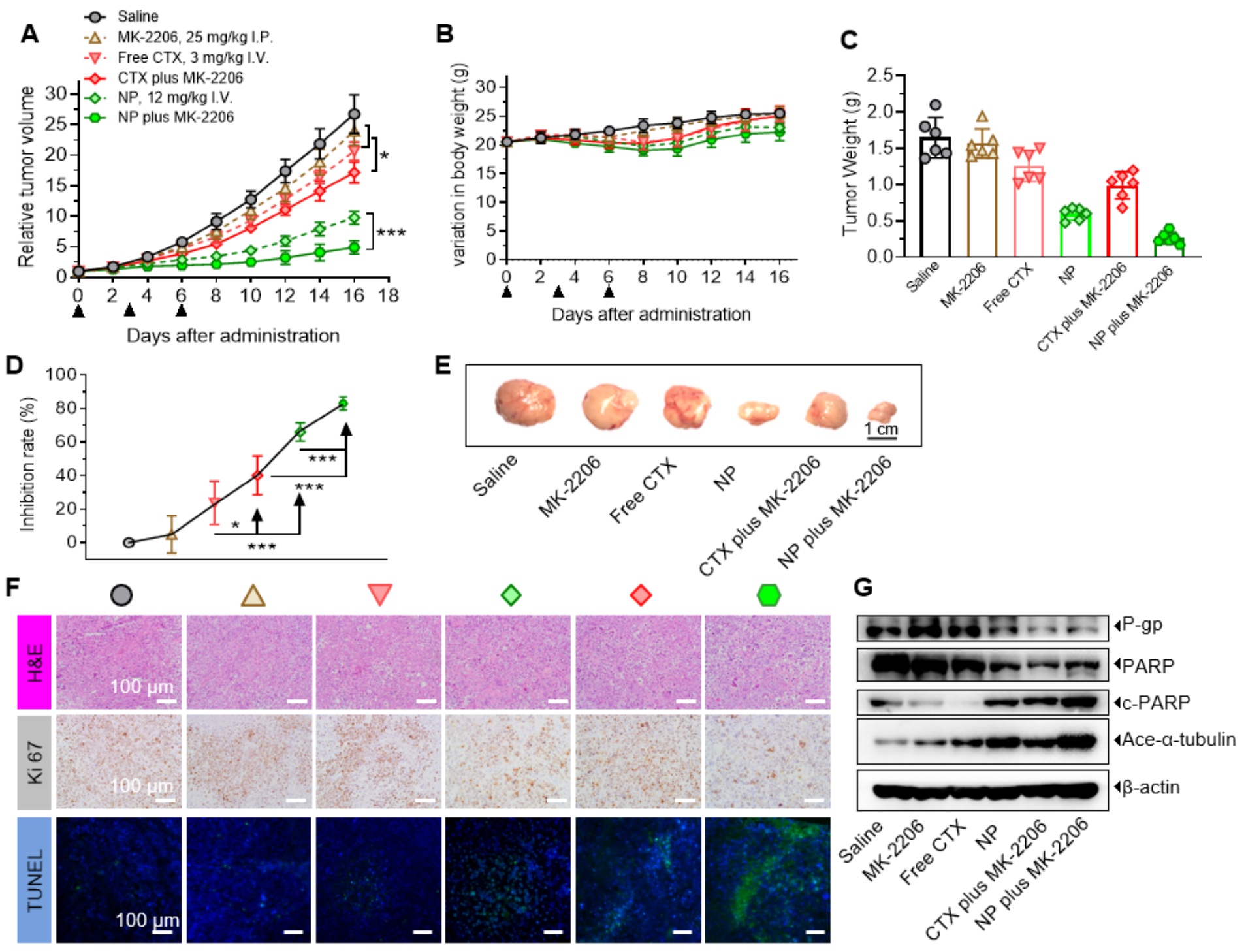

Figure 9

The combination of cabazitaxel-NPs and MK-2206 decreases tumor growth in HeLa/Taxol-bearing mice. a Tumor size changes during the treatments $(n=6)$. b Weight variations over time after various treatments $(n=6)$. Arrows indicate injection days. * indicates $p<0.05$, while $* \star \star$ indicates $p<0.001$ as determined by one-way ANOVA. c Tumor weights of each group at the endpoints. $d$ The tumor growth inhibition rate of each group was calculated according to tumor weights. e Representative images of tumors excised from different groups. Scale bar, $1 \mathrm{~cm}$. f Representative photographs of H\&E staining (upper), Ki-67 assay (middle), and TUNEL (lower) assay for tumor sections from different treatment groups at the endpoint. g Western blot results of P-gp, PARP, c-PARP, and acetyl-a-tubulin in isolated tumors.

\section{Supplementary Files}

This is a list of supplementary files associated with this preprint. Click to download. 
- Slwhx.docx

- SIwhx.docx

- Slwhx.docx 